\title{
Development of Motion Control Method for Laser Soldering Process
}

Federal Manufacturing \& Technologies

Scott S. Yerganian

KCP-613-5940

Published May 1997

Topical Report

P. M. Biggs, Project Leader
RECEVIDO

UUV 0907

OSTI

Approved for public release; distribution is unlimited.

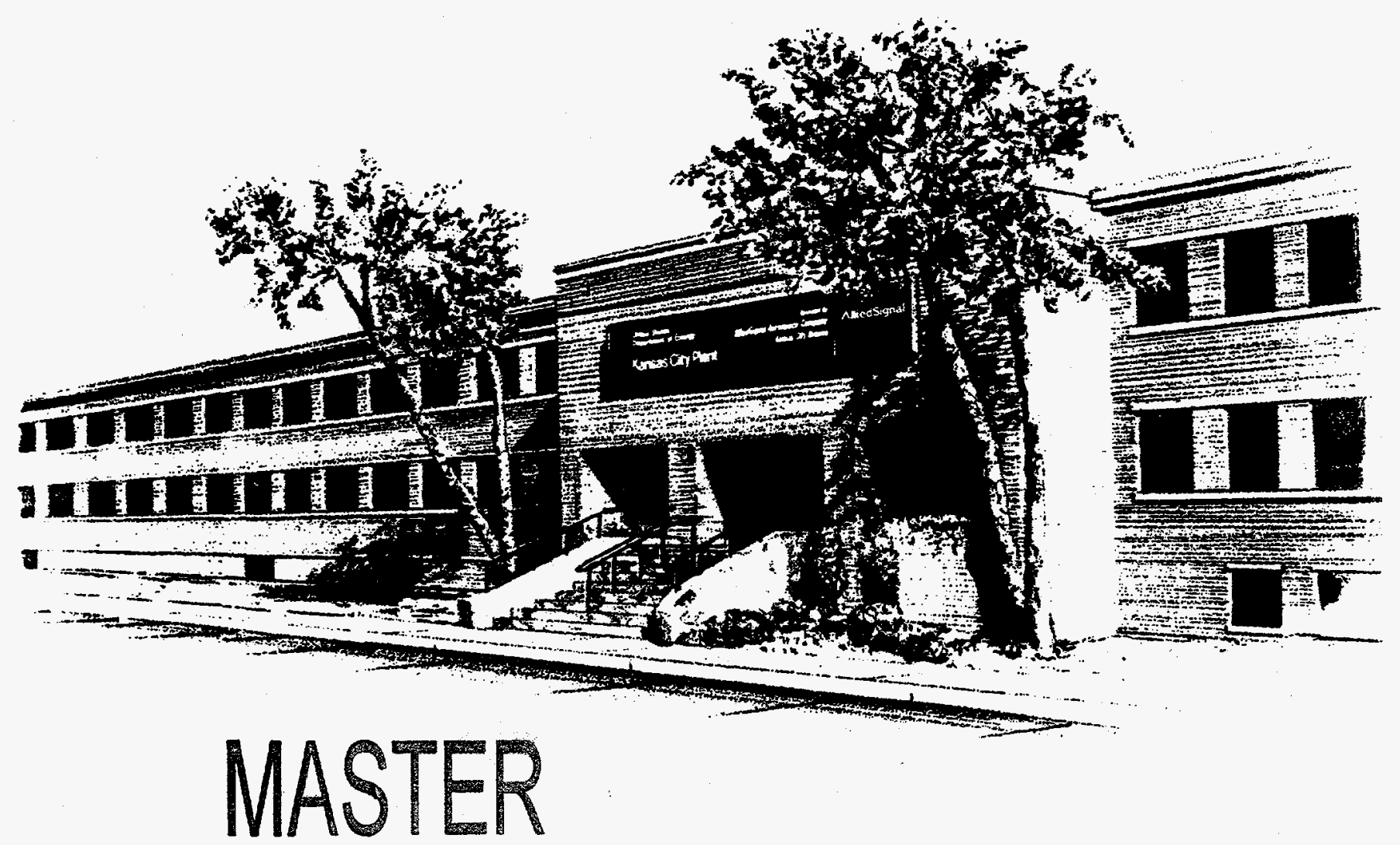

Prepared Under Contract Number DE-ACO4-76-DP00613 for the United States Department of Energy

AlliedSignal

A E R O S PA C E 


\section{DISCLAIMER}

This report was prepared as an account of work sponsored by an agency of the United States Government. Neither the United States Government nor any agency thereof, nor any of their employees, makes any warranty, express or implied, or assumes any legal liability or responsibility for the accuracy, completeness, or usefulness of any information, apparatus, product, or process disclosed, or represents that its use would not infringe privately owned rights. Reference herein to any specific commercial product, process, or service by trade names, trademark, manufacturer, or otherwise, does not necessarily constitute or imply its endorsement, recommendation, or favoring by the United States Government or any agency thereof. The views and opinions of authors expressed herein do not necessarily state or reflect those of the United States Government or any agency thereof.

Printed in the United States of America.

This report has been reproduced from the best available copy.

Available to DOE and DOE contractors from the Office of Scientific and Technical Information, P. O. Box 62, Oak Ridge, Tennessee 37831; prices available from (615) 576-8401, FTS 626-8401.

Available to the public from the National Technical Information Service, U. S. Department of Commerce, 5285 Port Royal Rd., Springfield, Virginia 22161.

AlliedSignal Inc. Fedleral Manufacturing \& Technologies P. O. Box 419159 Kansas City, Missouri 64141-6159 


\section{DISCLAIMIER}

Portions of this document may be illegible in electronic image products. Images are produced from the best available original doceoment. 
KCP-613-5940

Distribution Category UC-706

Approved for public release; distribution is unlimited.

\section{DEVELOPMENT OF MOTION CONTROL METHOD FOR LASER SOLDERING PROCESS}

Scott S. Yerganian

Published May 1997

Topical Report

P. M. Biggs, Project Leader 


\section{CONTENTS}

Section

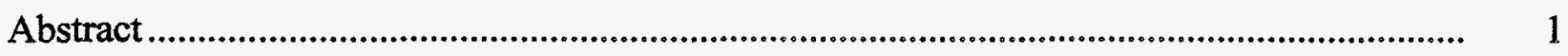

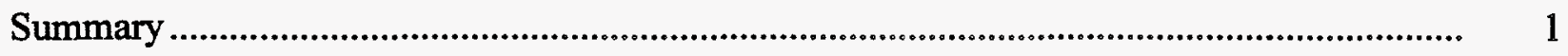

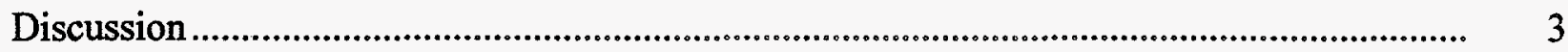

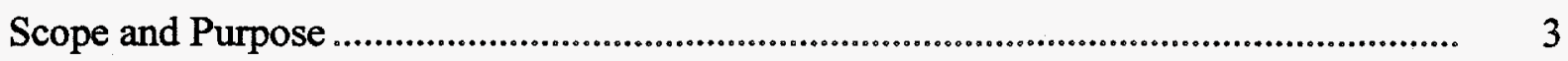

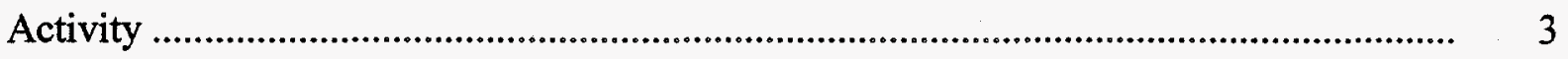

Motion Requirements.......................................................................................

Method for Generating Streaming Data to Drive the Motion Control System............ 3

Derivation of Velocity and Acceleration for Constant Travel Speed While Rotating Through Corner................................................................................................ 6

Method for Soldering Under Coaxial Cables............................................................... 10

Development of Spreadsheet and Macros.................................................................. 13

Simulation of Motion Using DADS ........................................................................ 21

Notes on Method Used to Verify Spreadsheet Data ..................................................... 22

Creating Curves and Drivers for Defining the Motion of the Hybrid and Laser Beam.

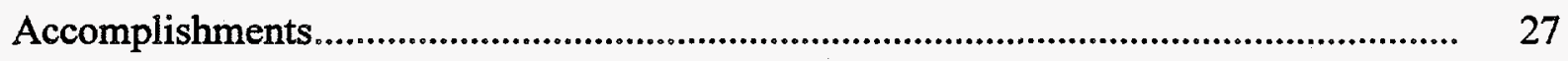

Appendix. Excel 5.0 Macros to Generate Motion Control Data.............................................. 28 


\section{ILLUSTRATIONS}

Figure

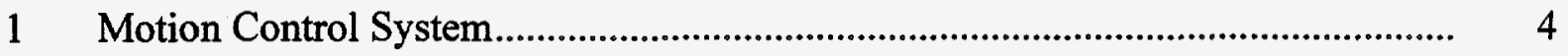

2 Positional Vectors of Part in Body Fixed and Global Coordinate Frames .............. 5

$3 \quad$ Motion Around Corner With Laser Perpendicular to Surface ............................... 7

4 Method of Rotating Part to Allow Laser to Solder Under Cable......................... 11

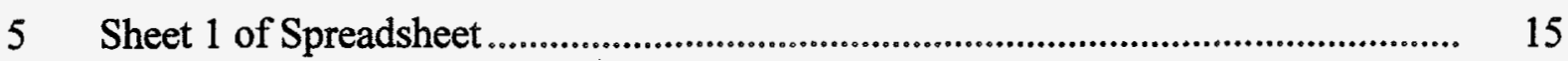

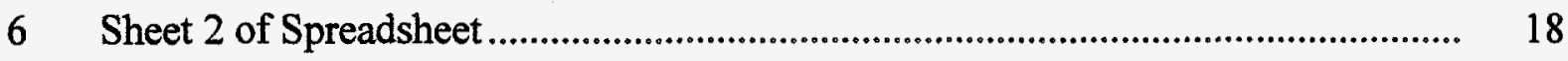

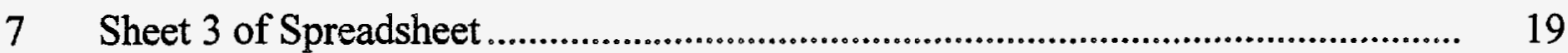

8 Motion Profile Data Created on Sheet 3 of Spreadsheet .................................... 20

$9 \quad$ Fourth Sheet of Spreadsheet Labeled "Setup Help" ${ }^{\prime \prime . . . . . . . . . . . . . . . . . . . . . . . . . . . . . . . . . . . . . . . . . . . . . ~} 21$

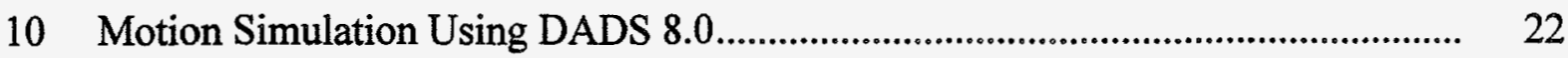




\section{ABSTRACT}

Development of a method to generate the motion control data for sealing an electronic housing using laser soldering is described. The motion required to move the housing under the laser is a nonstandard application and was performed with a four-axis system using the timed data streaming mode capabilities of a Compumotor AT6400 indexer. A Microsoft Excel 5.0 spreadsheet (named lsrauto.xls) was created to calculate the movement of the part under the laser, and macros were written into the spreadsheet to allow the user to easily create this data. A data verification method was developed for simulating the motion data. The geometry of the assembly was generated using Parametric Technology Corporation Pro/E version 15 . This geometry was then converted using Pro/DADS version 3.1 from Computer Aided Design Software Inc. (CADSI), and the simulation was carried out using DADS version 8.0 from CADSI.

\section{SUMMARY}

A method was developed for generating the motion of a radar housing under a laser for a laser soldering process. The purpose of this process is to solder a lid to a backing plate in order to seal the hybrid microelectronic circuit assembly from the environment. The design of this housing makes this a nonstandard application in which a two-dimensional table and common contouring motion control operation cannot be used because the laser is not along the axis of rotation of the part. Instead, the laser is along one of the translational axes, and the motion requires a method of driving the assembly under the laser in two dimensions while rotating about the assembly's center.

To further complicate matters, there are coaxial cables which get in the way and shade the assembly from the laser beam. This requires special movements to solder under the cables. To generate the motion, a four-axis system driven by a Compumotor AT6400 indexer was used. The software to program this indexer allows for a timed data streaming mode for nonstandard movements. In this mode, the user picks a streaming update interval, which may be any even number time interval from 10 milliseconds minimum to 50 milliseconds maximum. The user then supplies incremental move distances for each axis at each update point in time. In order to generate the data required for this streaming mode, a Microsoft Excel 5.0 spreadsheet (named lsrauto.xls) was created to calculate the movement of the part under the laser and convert this movement into the incremental step moves required. The amount of data to describe the motion becomes quite large, and macros were written into the spreadsheet to allow the user to easily create this data. A method was also developed for simulating the motion data. 
The geometry of the assembly was generated using Parametric Technology Corporation Pro/E version 15 . This geometry was then converted using Pro/DADS version 3.1 from Computer Aided Design Software Inc. (CADSI) and the simulation was carried out using DADS version 8.0 from CADSI. 


\section{DISCUSSION}

\section{SCOPE AND PURPOSE}

The purpose of this project was to develop a method for driving a radar housing containing a hybrid microelectronic circuit under a laser to solder it, sealing the assembly from the environment. A Microsoft Excel 5.0 spreadsheet was created to calculate the required movements of the part under the laser and to convert these movements into the incremental step moves required to drive the motion control system. The amount of data to describe the motion becomes quite large, and macros were written into the spreadsheet to allow the user to easily create this data. A method was also developed for simulating the motion data to verify the results of the spreadsheet calculations.

\section{ACTIVITY}

\section{Motion Requirements}

The design of the radar housing makes its movement under the laser a nonstandard application in which a two-dimensional table and common contouring motion control operation cannot be used because the laser is not along the axis of rotation of the part. Instead, the laser is along one of the translational axes and the motion requires a method of driving the assembly under the laser in two dimensions while rotating about the assembly's center (see Figure 1). To further complicate matters, there are coaxial cables that get in the way and shade the assembly from the laser beam. This requires special movements to solder under the cables. To generate the motion, a four-axis system driven by a Compumotor AT6400 indexer was used. The software to program this indexer allows for a timed data streaming mode for nonstandard movements. In this mode, the user picks a streaming update interval, which may be any even number time interval from 10 milliseconds minimum to 50 milliseconds maximum. The user then supplies incremental move distances for each axis at each update point in time.

\section{Method for Generating Streaming Data to Drive the Motion Control System}

The approach to generate the data for the motion control system was to allow the user to describe the desired motion around the part in the body fixed coordinate frame. This data was then to be transformed into incremental moves in the global coordinate frame required by the motion control system. Referring to Figure 2, the motion around the part is defined by the user in terms of the body fixed $\mathbf{S}$ ' vector. The components of this vector are the distances from the center of the part to the focal point of the laser, as the laser moves around the entire perimeter of the part. The focal point of the laser will always be on the outer surface of the part. Because of this, the 


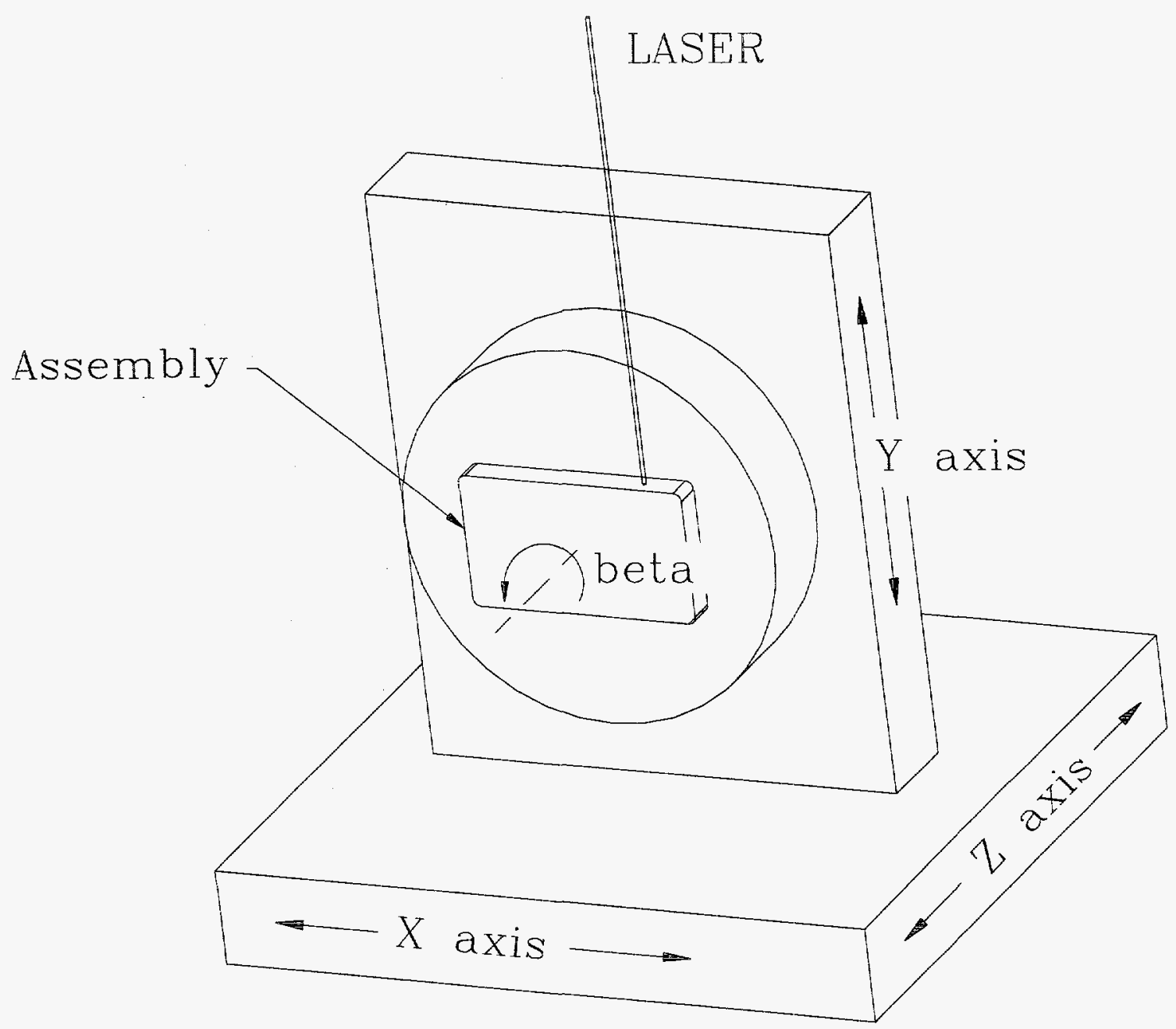

\section{Figure 1. Motion Control System}

S' vector (in body fixed coordinates) will have a constant y' coordinate (equal to plus or minus one half the width of the part) and vary in the $x^{\prime}$ direction, when the laser is shining on the top or bottom of the part. Likewise, when the laser is shining on the right or left sides of the part, the $S^{\prime}$ vector will have a constant $x^{\prime}$ coordinate (equal to plus or minus one half the length of the part) and vary in the $y^{\prime}$ direction. A transformation matrix is then used to convert the $\mathbf{S}^{\prime}$ vector in the body fixed coordinate frame to the $\mathbf{S}$ vector in global coordinates. The position of the focal point of the laser is labeled as the $\mathbf{r}_{\mathrm{p}}$ vector and is fixed at all times with respect to the global coordinate system. The position of the center of the part (at any point in time, in global coordinates) is labeled as the $\mathbf{r}_{\mathbf{A}}$ vector. This vector is found by subtracting the $\mathbf{S}$ vector from the $\mathbf{r}_{\mathbf{p}}$ vector. The incremental moves used to drive the motion control system are equal to the 


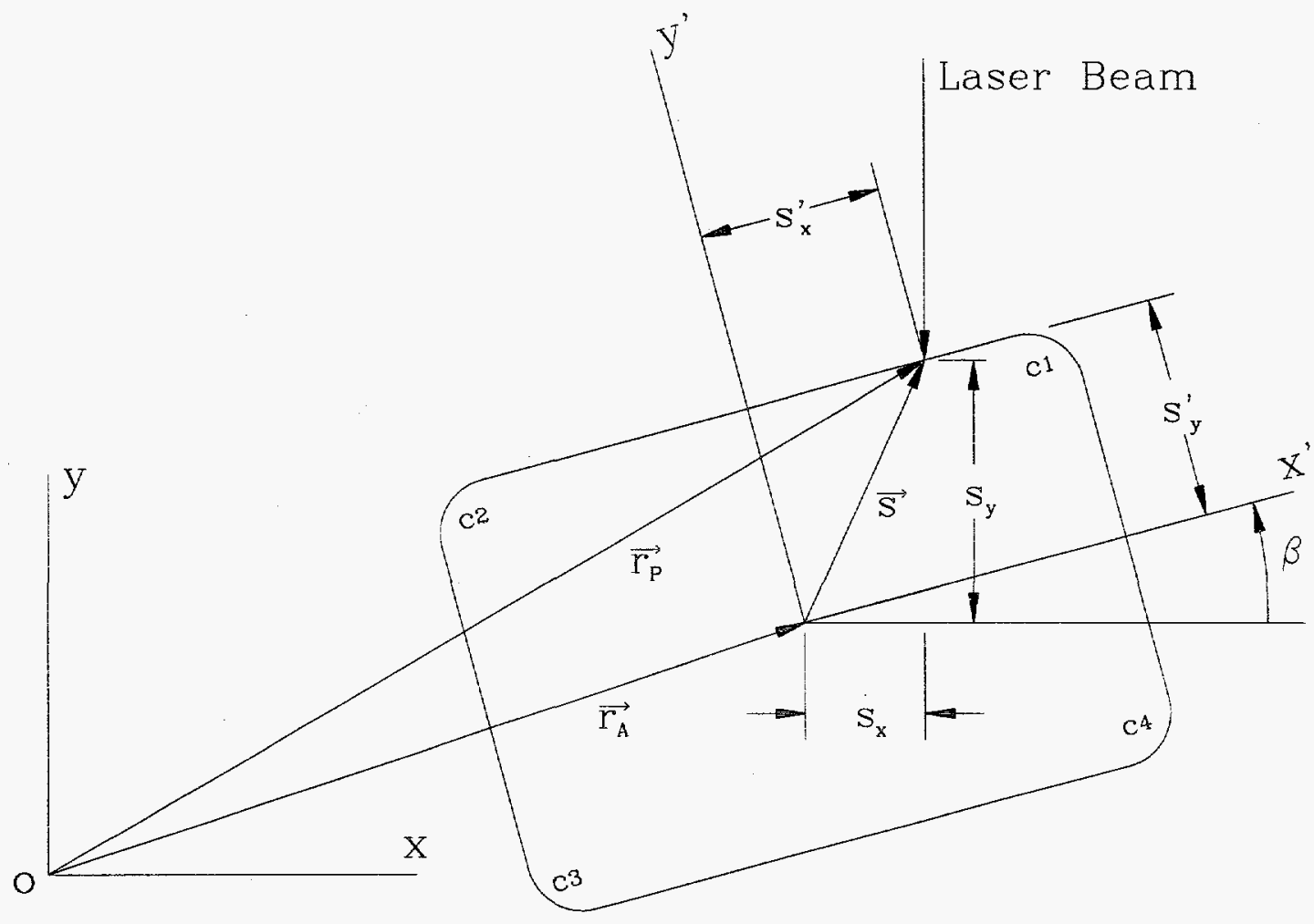

Figure 2. Positional Vectors of Part in Body Fixed and Global Coordinate Frames

changes in the global $\mathrm{x}$ and $\mathrm{y}$ components of the $\mathbf{r}_{\mathrm{A}}$ vector. Derivatives of the $\mathbf{S}$ vector were taken to determine the velocity and acceleration of the motion control system. If the velocities are too high, whipping of the lead screw will occur. If the accelerations are too high, the stepper or servo motors of the motion control system will not have enough power and torque to create the motion.

$\vec{s}^{\prime}=$ vector in body fixed coordinates from the part center to the focal point of the laser $\overrightarrow{\mathbf{s}}=A \overrightarrow{\mathbf{s}}^{\prime}$ where $\mathrm{A}$ is the transformation matrix

$A=\left[\begin{array}{cc}\cos \beta & -\sin \beta \\ \sin \beta & \cos \beta\end{array}\right]$ 
Expanding $\overrightarrow{\mathbf{s}}$ in terms of its components in the $\hat{\mathbf{i}}$ and $\hat{j}$ directions:

$$
\begin{aligned}
& \overrightarrow{\mathrm{s}}=\left(\mathrm{s}_{\mathrm{x}}^{\prime} \cos \beta-\mathrm{s}_{\mathrm{y}}^{\prime} \sin \beta\right) \hat{\mathrm{i}}+\left(\mathrm{s}_{\mathrm{x}}^{\prime} \sin \beta+\mathrm{s}_{\mathrm{y}}^{\prime} \cos \beta\right) \hat{\mathrm{j}} \\
& \overrightarrow{\mathrm{r}}_{\mathrm{A}}=\overrightarrow{\mathrm{r}}_{\mathrm{p}}-\overrightarrow{\mathrm{s}}
\end{aligned}
$$

Because $\vec{r}_{p}$ is a fixed point in the global coordinate system, the velocity of the center of the part $\dot{\overrightarrow{\mathrm{r}}}_{\mathrm{A}}=-\dot{\overrightarrow{\mathrm{s}}}$. Likewise, the acceleration of the center of the part $\ddot{\overrightarrow{\mathrm{r}}}_{\mathrm{A}}=-\ddot{\overrightarrow{\mathrm{s}}}$.

Taking the derivatives of (equation 1) to find the general relationship for the part velocity:

$$
\begin{array}{r}
\dot{\overrightarrow{\mathrm{s}}}=\left(-\mathrm{s}_{\mathrm{x}}^{\prime} \dot{\beta} \sin \beta+\dot{\mathrm{s}}_{\mathrm{x}}^{\prime} \cos \beta-\mathrm{s}_{\mathrm{y}}^{\prime} \dot{\beta} \cos \beta-\dot{\mathrm{s}}_{\mathrm{y}}^{\prime} \sin \beta\right) \hat{\mathrm{i}} \\
+ \\
\left(\mathrm{s}_{\mathrm{x}}^{\prime} \dot{\beta} \cos \beta+\dot{\mathrm{s}}_{\mathrm{x}}^{\prime} \sin \beta-\mathrm{s}_{\mathrm{y}}^{\prime} \dot{\beta} \sin \beta+\dot{\mathrm{s}}_{\mathrm{y}}^{\prime} \cos \beta\right) \hat{\mathrm{j}}
\end{array}
$$

Differentiating (equation 2) to find the general relationship for the part acceleration:

$$
\begin{aligned}
\ddot{\vec{s}}= & \left(-s_{x}^{\prime} \dot{\beta}^{2} \cos \beta-s_{x}^{\prime} \ddot{\beta} \sin \beta-2 \dot{s}_{x}^{\prime} \dot{\beta} \sin \beta+\ddot{s}_{x}^{\prime} \cos \beta+s_{y}^{\prime} \dot{\beta}^{2} \sin \beta-s_{y}^{\prime} \ddot{\beta} \cos \beta-2 \dot{s}_{y}^{\prime} \dot{\beta} \cos \beta-\ddot{s}_{y}^{\prime} \sin \beta\right) \hat{\mathrm{i}} \\
& + \\
& \left(-s_{x}^{\prime} \dot{\beta}^{2} \sin \beta+s_{x}^{\prime} \ddot{\beta} \cos \beta+2 \dot{s}_{x}^{\prime} \dot{\beta} \cos \beta+\ddot{s}_{x}^{\prime} \sin \beta-s_{y}^{\prime} \dot{\beta}^{2} \cos \beta-s_{y}^{\prime} \ddot{\beta} \sin \beta-2 \dot{s}_{y}^{\prime} \dot{\beta} \sin \beta+\ddot{s}_{y}^{\prime} \cos \beta\right) \hat{j}
\end{aligned}
$$

(equation 3)

\section{Derivation of Velocity and Acceleration for Constant Travel Speed While Rotating Through Corner}

If the laser is to move at a constant travel speed, remain perpendicular to the edge of the part, and the part rotations begin and end with the beginning and ending of each corner, the velocities and accelerations in the corners become very large. The smaller the corner radius, the higher these velocities and accelerations become. The reason for this is that, for this type of motion, the angular velocity around the corner is the travel speed (tangential velocity) divided by the radius. For a constant travel velocity, this angular velocity, and consequently the velocities of the part to correct for the off-radius rotation, become larger as the radius becomes smaller. In the limit, the velocities and accelerations approach infinity for a zero radius (perfectly sharp corner). Deriving the magnitude of the part velocity and accelerations, for this motion in a corner, as a function of the travel speed and part geometry (referring to Figure 3): 


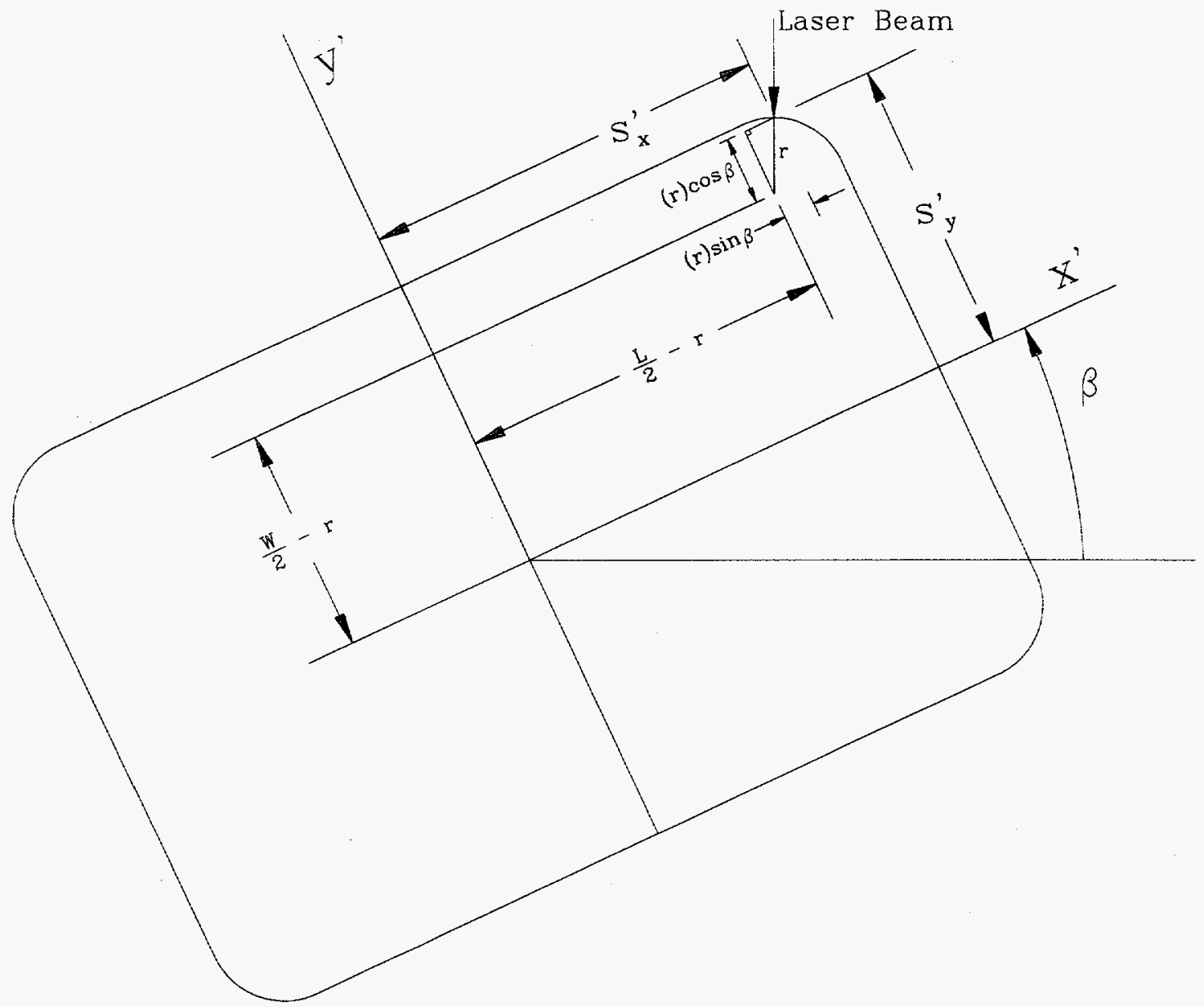

Figure 3. Motion Around Corner With Laser Perpendicular to Surface

$$
\begin{aligned}
& \mathrm{s}_{\mathrm{x}}^{\prime}=\frac{\mathrm{L}}{2}-\mathrm{r}+\mathrm{r} \sin \beta \\
& \mathrm{s}_{\mathrm{y}}^{\prime}=\frac{\mathrm{W}}{2}-\mathrm{r}+\mathrm{r} \cos \beta \\
& \dot{\mathrm{s}}_{\mathrm{x}}^{\prime}=\mathrm{r} \dot{\beta} \cos \beta \\
& \dot{\mathrm{s}}_{\mathrm{y}}^{\prime}=-\mathrm{r} \dot{\beta} \sin \beta
\end{aligned}
$$

(equation 7) 
For constant travel speed $\mathrm{V}$ around the corner:

$\dot{\beta}=\frac{V}{r} \quad$ and $\quad \ddot{\beta}=0$

Plugging the above relationships for $\dot{\beta}$ into (equations 6 and 7):

$\dot{\mathrm{s}}_{\mathrm{x}}^{\prime}=\mathrm{V} \cos \beta$

(equation 8)

$\dot{\mathrm{s}}_{\mathrm{y}}^{\prime}=-\mathrm{V} \sin \beta$

(equation 9)

Plugging the relationship $\dot{\beta}=\frac{V}{r}$ and (equations 8 and 9) into (equation 2):

$\begin{aligned} \dot{\overrightarrow{\mathrm{s}}}= & {\left[-\left(\frac{\mathrm{L}}{2}-\mathrm{r}+\mathrm{r} \sin \beta\right) \frac{\mathrm{V}}{\mathrm{r}} \sin \beta+\mathrm{V} \cos ^{2} \beta-\left(\frac{\mathrm{W}}{2}-\mathrm{r}+\mathrm{r} \cos \beta\right) \frac{\mathrm{V}}{\mathrm{r}} \cos \beta+\mathrm{V} \sin ^{2} \beta\right] \hat{\mathrm{i}} } \\ + & {\left[\left(\frac{\mathrm{L}}{2}-\mathrm{r}+\mathrm{r} \sin \beta\right) \frac{\mathrm{V}}{\mathrm{r}} \cos \beta+\mathrm{V} \cos \beta \sin \beta-\left(\frac{\mathrm{W}}{2}-\mathrm{r}+\mathrm{r} \cos \beta\right) \frac{\mathrm{V}}{\mathrm{r}} \sin \beta-\mathrm{V} \sin \beta \cos \beta\right] \hat{\mathrm{j}} }\end{aligned}$

Simplifying and using the identity $\sin ^{2} \beta+\cos ^{2} \beta=1$ :

$$
\begin{aligned}
\dot{\vec{s}}= & {\left[-\frac{\mathrm{V}}{\mathrm{r}}\left\{\left(\frac{\mathrm{L}}{2}-\mathrm{r}\right) \sin \beta+\left(\frac{\mathrm{W}}{2}-\mathrm{r}\right) \cos \beta\right\} \hat{\mathrm{i}}\right.} \\
+ & {\left[\frac{\mathrm{V}}{\mathrm{r}}\left\{\left(\frac{\mathrm{L}}{2}-\mathrm{r}\right) \cos \beta-\left(\frac{\mathrm{W}}{2}-\mathrm{r}\right) \sin \beta\right\}\right] \hat{\mathrm{j}} }
\end{aligned}
$$


Differentiating (equation 10) to find the acceleration, and using the relationship $\dot{\beta}=\frac{V}{r}$ :

$$
\begin{aligned}
\ddot{\overrightarrow{\mathrm{s}}}= & {\left[-\left(\frac{\mathrm{V}}{\mathrm{r}}\right)^{2}\left\{\left(\frac{\mathrm{L}}{2}-\mathrm{r}\right) \cos \beta-\left(\frac{\mathrm{W}}{2}-\mathrm{r}\right) \sin \beta\right\} \hat{\mathrm{i}}\right.} \\
& + \\
& {\left[-\left(\frac{\mathrm{V}}{\mathrm{r}}\right)^{2}\left\{\left(\frac{\mathrm{L}}{2}-\mathrm{r}\right) \sin \beta+\left(\frac{\mathrm{W}}{2}-\mathrm{r}\right) \cos \beta\right\}\right] \hat{\mathrm{j}} }
\end{aligned}
$$

The maximum velocity in the corner occurs when its derivative is zero. For the non-trivial solution, the bracketed term of (equation 11) in the $\mathrm{x}$ and $\mathrm{y}$ direction are set equal to zero:

$\dot{\mathrm{s}}_{\mathrm{x} \max }$ occurs when $\left\{\left(\frac{\mathrm{L}}{2}-\mathrm{r}\right) \cos \beta-\left(\frac{\mathrm{W}}{2}-\mathrm{r}\right) \sin \beta\right\}=0$ or $\tan \beta=\frac{\left(\frac{\mathrm{L}}{2}-\mathrm{r}\right)}{\left(\frac{\mathrm{W}}{2}-\mathrm{r}\right)}$

(equation 12)

$\dot{\mathrm{s}}_{\mathrm{y} \max }$ occurs when $\left\{\left(\frac{\mathrm{L}}{2}-\mathrm{r}\right) \sin \beta+\left(\frac{\mathrm{W}}{2}-\mathrm{r}\right) \cos \beta\right\}=0$ or $-\tan \beta=\frac{\left(\frac{\mathrm{W}}{2}-\mathrm{r}\right)}{\left(\frac{\mathrm{L}}{2}-\mathrm{r}\right)}$ (equation 13)

The maximum acceleration in the corner occurs when its derivative is zero. Taking the derivative of (equation 11) and using the relationship $\dot{\beta}=\frac{V}{r}$ :

$$
\begin{aligned}
\ddot{\vec{s}}= & {\left[\left(\frac{\mathrm{V}}{\mathrm{r}}\right)^{3}\left\{\left(\frac{\mathrm{L}}{2}-\mathrm{r}\right) \sin \beta+\left(\frac{\mathrm{W}}{2}-\mathrm{r}\right) \cos \beta\right\} \hat{\mathrm{i}}\right.} \\
& {\left[-\left(\frac{\mathrm{V}}{\mathrm{r}}\right)^{3}\left\{\left(\frac{\mathrm{L}}{2}-\mathrm{r}\right) \cos \beta-\left(\frac{\mathrm{W}}{2}-\mathrm{r}\right) \sin \beta\right\}\right] \hat{\mathrm{j}} }
\end{aligned}
$$


For the non-trivial solution, the bracketed term of the components in the $\mathrm{x}$ and $\mathrm{y}$ direction of (equation 14) are set equal to zero:

$\ddot{\mathrm{s}}_{\mathrm{x} \max }$ occurs when $\left\{\left(\frac{\mathrm{L}}{2}-\mathrm{r}\right) \sin \beta+\left(\frac{\mathrm{W}}{2}-\mathrm{r}\right) \cos \beta\right\}=0 \quad$ or $\quad-\tan \beta=\frac{\left(\frac{\mathrm{W}}{2}-\mathrm{r}\right)}{\left(\frac{\mathrm{L}}{2}-\mathrm{r}\right)}$

(equation 15)

$\ddot{\mathrm{s}}_{\mathrm{y} \max }$ occurs when $\left\{\left(\frac{\mathrm{L}}{2}-\mathrm{r}\right) \cos \beta-\left(\frac{\mathrm{W}}{2}-\mathrm{r}\right) \sin \beta\right\}=0$ or $\tan \beta=\frac{\left(\frac{\mathrm{L}}{2}-\mathrm{r}\right)}{\left(\frac{\mathrm{W}}{2}-\mathrm{r}\right)}$

(equation 16)

The use of these relationships allows the user to determine the requirements on a motion control system if this motion is desired in the corners. To find the maximum velocities through the corner in global coordinates, equation 10 is evaluated at the angles described by equations 12 and 13. To find the maximum accelerations through the corner in global coordinates, equation 11 is evaluated at the angles described by equations 15 and 16.

The demands on the motion control system, for a part with a small corner radius, may be significantly reduced, if the constraint of holding the part perpendicular to the laser is relaxed when nearing and exiting a corner. This allows the rotation of the part to be spread over a greater distance (beginning rotation at a small distance before reaching the corner radius, and ending rotation at a small distance after the corner radius).

\section{Method for Soldering Under Coaxial Cables}

A method was developed to allow the laser to solder under the area shaded by a cable. In this method, the laser is brought up to the edge of the cable diameter, or the diameter of a tolerance "keep out" zone around the cable. The edge of the laser then "hugs" this diameter as the part is rotated, to allow the beam to move under the cable. Since the angle beta is used to describe the absolute rotation of the part, the angle alpha is used to describe the angle of the laser as it moves under the cable. The angle alpha must be solved for as a function of the distance of the centerline of the laser under the cable. The absolute rotation beta of the part is then either increased or decreased by the alpha angle, depending on the direction of movement around the part. For deriving the angle alpha as the laser is moved under a cable, see Figure 4. 


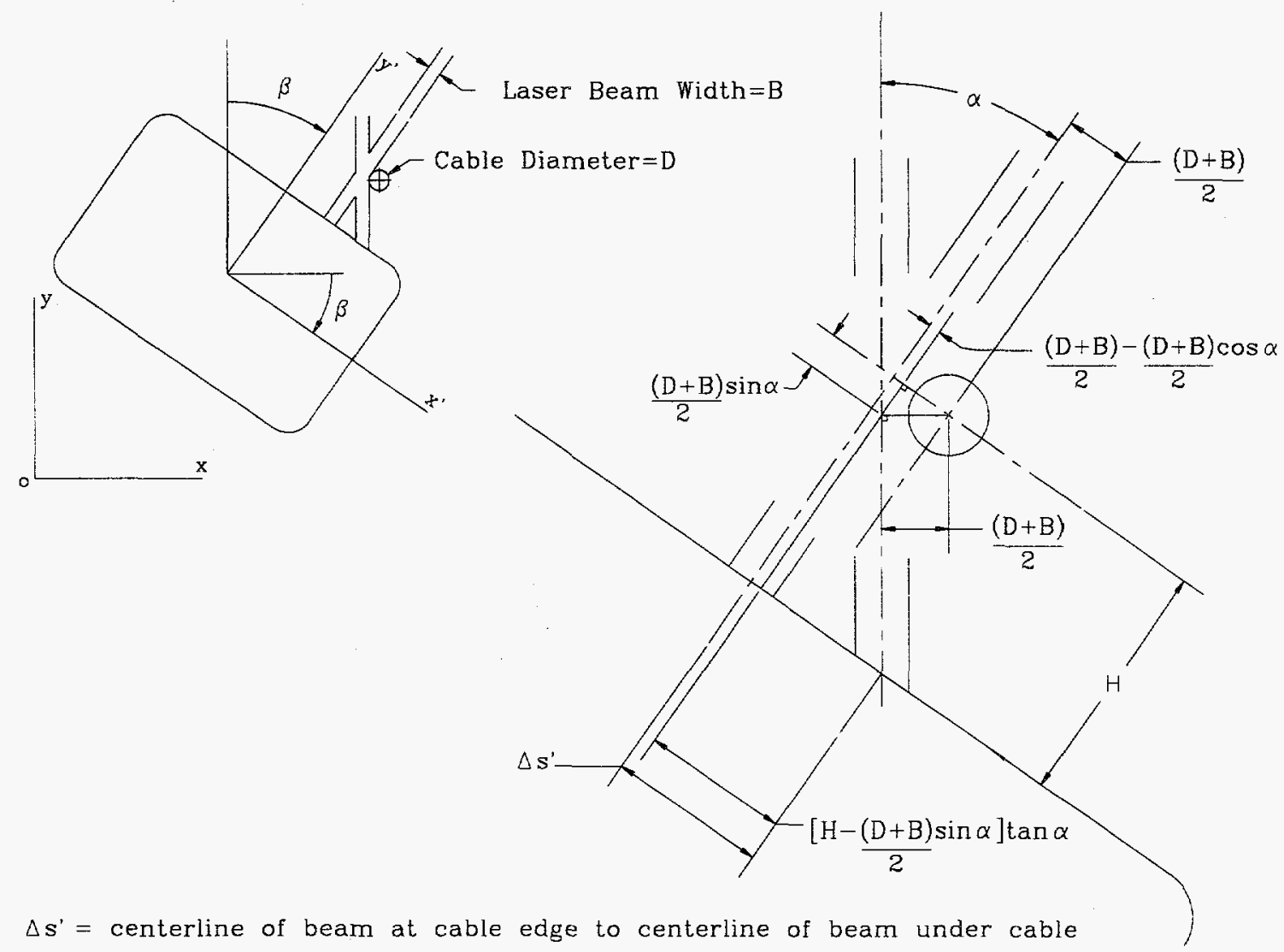

Figure 4. Method of Rotating Part to Allow Laser to Solder Under Cable

From the distances defined in Figure 4:

$\Delta \mathrm{s}=\left(\frac{\mathrm{D}+\mathrm{B}}{2}\right)-\left(\frac{\mathrm{D}+\mathrm{B}}{2}\right) \cos \alpha+\left(\mathrm{H}-\left(\frac{\mathrm{D}+\mathrm{B}}{2}\right) \sin \alpha\right) \tan \alpha$

Writing the $\tan \alpha$ as the $\frac{\sin \alpha}{\cos \alpha}$ :

$\Delta \mathrm{s}=\left(\frac{\mathrm{D}+\mathrm{B}}{2}\right)-\left(\frac{\mathrm{D}+\mathrm{B}}{2}\right) \cos \alpha+\mathrm{H} \frac{\sin \alpha}{\cos \alpha}-\left(\frac{\mathrm{D}+\mathrm{B}}{2}\right) \frac{\sin ^{2} \alpha}{\cos \alpha}$ 
Multiplying both sides by $\cos \alpha$ :

$\Delta \mathrm{s} \cos \alpha=\left(\frac{\mathrm{D}+\mathrm{B}}{2}\right) \cos \alpha-\left(\frac{\mathrm{D}+\mathrm{B}}{2}\right) \cos ^{2} \alpha+\mathrm{H} \sin \alpha-\left(\frac{\mathrm{D}+\mathrm{B}}{2}\right) \sin ^{2} \alpha$

Using the identity $\sin ^{2} \alpha+\cos ^{2} \alpha=1$ :

$\Delta \mathrm{s} \cos \alpha=\left(\frac{\mathrm{D}+\mathrm{B}}{2}\right) \cos \alpha-\left(\frac{\mathrm{D}+\mathrm{B}}{2}\right)+\mathrm{H} \sin \alpha$

Rearranging terms:

$\left[\Delta \mathrm{s}-\left(\frac{\mathrm{D}+\mathrm{B}}{2}\right)\right] \cos \alpha+\left(\frac{\mathrm{D}+\mathrm{B}}{2}\right)=\mathrm{H} \sin \alpha$

Squaring both sides:

$\left[\Delta s-\left(\frac{D+B}{2}\right)\right]^{2} \cos ^{2} \alpha+2\left(\frac{D+B}{2}\right)\left[\Delta s-\left(\frac{D+B}{2}\right)\right] \cos \alpha+\left(\frac{D+B}{2}\right)^{2}=H^{2} \sin ^{2} \alpha$

Using the identity $\sin ^{2} \alpha=1-\cos ^{2} \alpha$ :

$\left[\Delta \mathrm{s}-\left(\frac{\mathrm{D}+\mathrm{B}}{2}\right)\right]^{2} \cos ^{2} \alpha+2\left(\frac{\mathrm{D}+\mathrm{B}}{2}\right)\left[\Delta \mathrm{s}-\left(\frac{\mathrm{D}+\mathrm{B}}{2}\right)\right] \cos \alpha+\left(\frac{\mathrm{D}+\mathrm{B}}{2}\right)^{2}=\mathrm{H}^{2}-\mathrm{H}^{2} \cos ^{2} \alpha$

Rearranging:

$\left\{\left[\Delta \mathrm{s}-\left(\frac{\mathrm{D}+\mathrm{B}}{2}\right)\right]^{2}+\mathrm{H}^{2}\right\} \cos ^{2} \alpha+\left\{2\left(\frac{\mathrm{D}+\mathrm{B}}{2}\right)\left[\Delta \mathrm{s}-\left(\frac{\mathrm{D}+\mathrm{B}}{2}\right)\right]\right\} \cos \alpha+\left\{\left(\frac{\mathrm{D}+\mathrm{B}}{2}\right)^{2}-\mathrm{H}^{2}\right\}=0$

This equation is now a quadratic equation of the form $a^{2}+b x+c=0$ where $x=\cos \alpha$ and may be solved using the quadratic formula $x=\frac{-b \pm \sqrt{b^{2}-4 a c}}{2 a}$ (with the angle alpha equal to the arc cosine of the solution) where:

$\mathrm{a}=\left\{\left[\Delta \mathrm{s}-\left(\frac{\mathrm{D}+\mathrm{B}}{2}\right)\right]^{2}+\mathrm{H}^{2}\right\}$ 


$$
\begin{aligned}
& \mathrm{b}=\left\{2\left(\frac{\mathrm{D}+\mathrm{B}}{2}\right)\left[\Delta \mathrm{s}-\left(\frac{\mathrm{D}+\mathrm{B}}{2}\right)\right]\right\} \\
& \mathrm{c}=\left\{\left(\frac{\mathrm{D}+\mathrm{B}}{2}\right)^{2}-\mathrm{H}^{2}\right\}
\end{aligned}
$$

\section{Development of Spreadsheet and Macros}

Because the data streaming mode used with the motion control system requires incremental move data every 10 to 50 milliseconds (depending on the streaming update interval chosen), the amount of data required for the full motion of the housing under the laser becomes quite large. A Microsoft Excel 5.0 spreadsheet was created containing macros to enable a user to easily generate the data required for the streaming mode of the motion control system. The macros of this spreadsheet are contained within three modules which are included in the Appendix. The macros take advantage of the method discussed earlier for converting the body fixed coordinates of the part to the incremental moves required by the motion control system. They also incorporate the solution of the quadratic equation used to define movements under the cables.

The spreadsheet (called lsrauto.xls) consists of four sheets and three modules of macros written in the Visual Basic version contained within the Excel 5.0 program. To make use of the spreadsheet, the user must also have two additional Excel files in the same directory that contain the lsrauto.xls file. These additional files are labeled dads_dat.xls and mtn_dad.csv. The dads_dat.xls file is an Excel 5.0 spreadsheet containing only one sheet. The macros of the lsrauto.xls spreadsheet open this spreadsheet and use it to create the rax.txt, ray.txt, beta.txt, and shutter.txt files required to perform the DADS simulation. The mtn_dad.csv file is the comma delimited file data used to drive the motion control system. The macros of the lsrauto.xls spreadsheet open this file, to create the required file. The user may then rename the file when saving to another desired file name for the data.

The approach used in the spreadsheet and macros is for the user to define the minimum data required for the desired motion of the part under the laser on the first sheet of the spreadsheet. The macros then expand upon this data in creating the second and third sheets. The definition data supplied by the user consists of the major points around the part geometry where an event is to occur. Examples of the points that the user defines on the first sheet are the starting and ending points, the corners, the cables, and any other points in which a specific travel speed under the laser or part rotation angle is desired. To make this definition easier for the user, the method for defining the moves through corners and under cables on the first sheet of the spreadsheet was simplified. The user simply makes a notation designating the corner number to define a corner move. Likewise, the user provides the minimum data required to define a cable move. The macros within the spreadsheet copy the data on the first sheet and create a second sheet where the 
movements through the corners and under the cables are more fully defined. The only thing lacking on the second sheet for the full definition of the moves through the corners and under the cables is the desired travel speed under the laser. The user must then edit the second sheet to include the desired travel speed for these few additional points around the part. It is important to note that the user must make sure that all rows of data on the second sheet contain values for the travel speed but that all rows do not require values for the angle beta.

Because the motion definition thus far is only of some major points around the part, the spreadsheet must now generate the large amount of data that defines each incremental move at every time step that occurs between any two major points. This full definition of the entire motion around the part is performed on the third sheet of the spreadsheet. The data on the third sheet is entirely created by the macros of the spreadsheet. The macros within the spreadsheet copy the data on the second sheet and create the third sheet.

To generate the full data that exists on the third sheet, macros scan down the data copied over from the second sheet and insert rows to define all of the incremental moves that exist between each pair of major points that the user defined on the first and second sheets. Each pair of major points defined by the user is treated as an interval in which the starting and ending coordinates and travel speed are defined. To create the data for the rows inserted within this interval, the travel speeds defined by the user at the beginning and at the end of the interval are compared. The macros then change the travel speed from the beginning value to the ending value linearly over the interval (constant acceleration or deceleration).

There is a slight difference in the way that changes in the rotation angle beta for the part are handled. Once the macros have inserted all of the additional rows, into the third sheet, for the entire motion around the part, the macros will go back and scan through the data to generate the values for the beta angle. A macro counts the number of rows, not containing beta values, existing in an interval between any two defined beta values. It then changes the beta angle from the beginning value to the ending value linearly over the interval. The advantage to this method is that the user is not required to define a beta angle for most of the major points defined on the first and second sheets. The user then only defines a beta angle at a point in which a specific value is desired. The macros that create the third sheet will then gradually vary the beta angle over all of the moves that exist between any two defined beta values.

This method is especially useful to allow a gradual rotation around a corner. To do this, the user defines a point on the first sheet where the start of rotation is desired before a corner. The beta angle at this point is defined as the angle before rotation (the last point that will have this beta angle). The user then defines the corner on the first sheet using the corner notation. Finally, the user defines a point on the first sheet where the end of rotation is desired after the corner. The beta value at this point is the angle at the end of rotation. A similar "scan and fill in" method is used for the shutter values on the third sheet. The macro that fills in the shutter values on the third sheet simply scans down the rows of inserted data and fills in any missing shutter value as 
the last value previously defined. Because of this, the user only needs to fill in a value in the shutter column at the beginning and end of the data on the first and second sheets, and at any other point in which a change in shutter value is desired.

\section{Sheet 1}

Sheet 1 of the lsrauto.xls spreadsheet (Figure 5) contains the basic part and motion definition data that the user creates to define the desired motion. Once this data is defined, the user makes a mouse click on the button labeled "Expand User Input Data on Sheet 2." This runs the macros that delete the old sheet 2 and sheet 3 and creates a new sheet 2 .

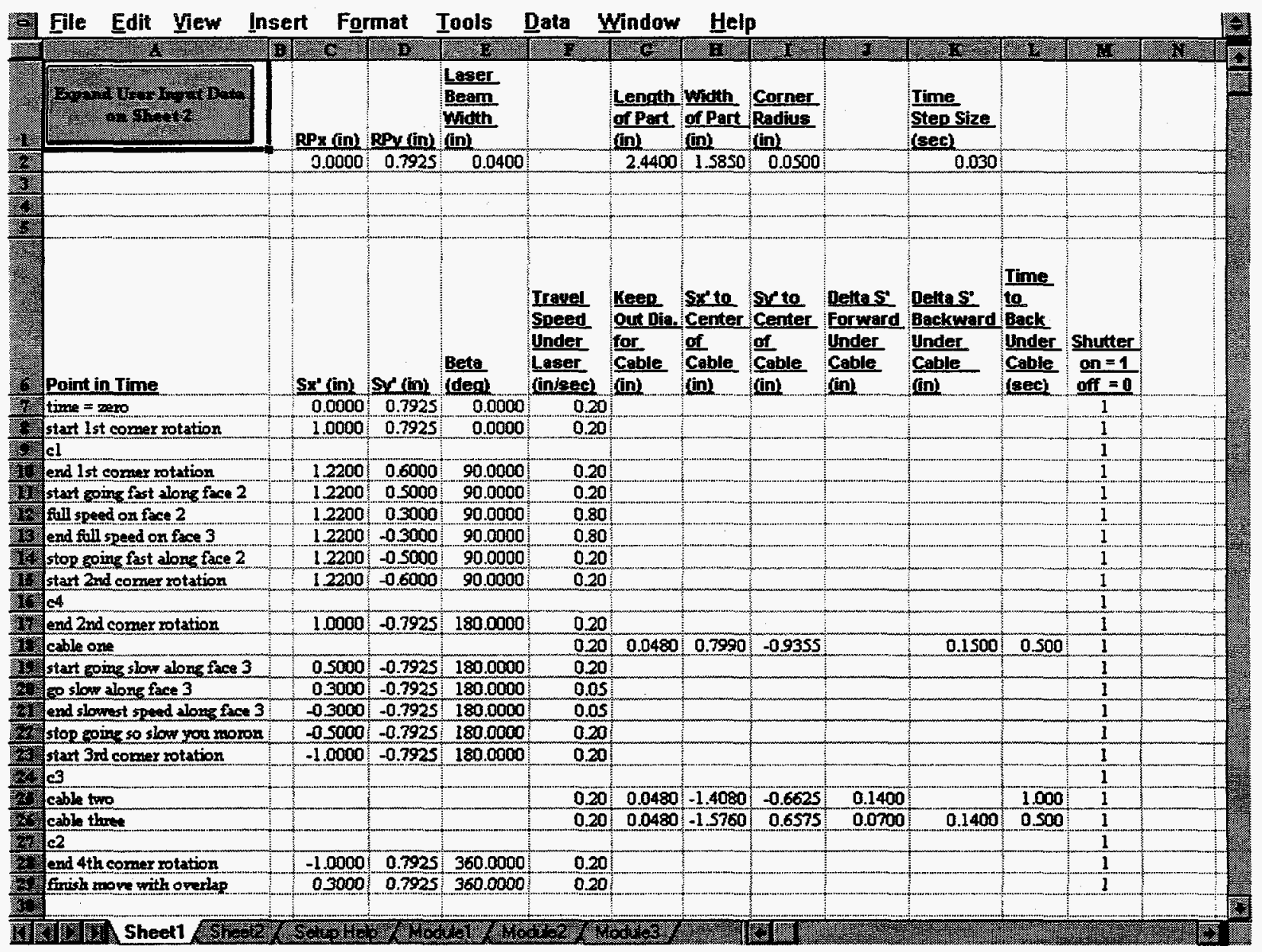

\section{Figure 5. Sheet 1 of Spreadsheet}

\section{Summary of Definition From User on the First Sheet:}

- Starting point definition

- Corners (defined by corner designator $\mathrm{c} 1, \mathrm{c} 2, \mathrm{c} 3$, or $\mathrm{c4}$ ) 
- Cables (defined by definitions in cable data fields)

- Definition of other points to make changes in travel speed or angle

- Ending point definition

\section{Sheet 1 Definition Notes:}

1. The user must define a starting point as the first line of data that includes sx', sy', beta, travel speed, and shutter values. For this entry, any notation may be written, if desired, in the "Point in Time" field. The only exception to defining the starting point in this manner would be if the user specifically wants to start the motion at a corner or cable. To do this, the first line of data would be a corner or cable definition.

2. All points defined on Sheet 1 must be in the sequential order that the movement is to occur around the part. The user may define a movement clockwise or counterclockwise around the part. The beta angles defined are always positive angles from 0 to 360 degrees. If the part is to rotate counterclockwise under the laser, the beta angle, at the first point defined, will usually be 0 degrees, with 360 degrees as the angle for last point defined. If the part is to rotate clockwise under the laser, the beta angle will usually start at 360 degrees and end at 0 degrees.

3. To define a corner, all fields are left blank, with the exception that the corner designator $\mathrm{cl}$, c2, c3, or c4, must be typed into the "Point in Time" field. This designator must be typed in exactly including using the lowercase letter " $\mathrm{c}$ ".

4. To define a cable, the sx', sy', and beta fields are left blank. The travel speed definition is optional. The user must define the size of the "keep out diameter" around the cable, the sx' and sy' coordinates to the center of the cable, the distance for the laser to move forward under the cable (if desired), the distance for the laser to move backward under the cable (if desired), and the time in which the shutter turns the laser off while the part is moved and rotated into a position on the other side of the cable. The user may have the laser move both forward and backward under the cable by filling in the distances under the respective columns, but at least one of these distances (forward or backward) must be defined. For a cable entry, any notation may be written, if desired, in the "Point in Time" field.

5. Any other point may be defined, as long as it is in the sequential order that the movement is to occur around the part. Examples of such points included in the motion definition include points at which a specific velocity or angle is desired. To define such a point, the sx', sy', and travel speed values are required. Values for beta and shutter are optional.

6. The user must define an ending point as the last line of data that includes sx', sy', beta, travel speed, and shutter values. For this entry, any notation may be written, if desired, in the "Point in Time" field. The only exception to defining the ending point in this manner would be if the user specifically wants to end the motion at a corner or cable. To do this, the last line of data would be a corner or cable definition. 
7. The macros were made general enough to allow the travel speed around the part to increase or decrease over any interval (including movements under cables or movements around corners). To accomplish this, constant acceleration or deceleration is used in an interval (the velocity is linearly ramped up or down).

\section{Sheet 2}

Sheet 2 (see Figure 6) is a copy of sheet 1 that is then expanded to include additional definition data required for movement around the corners and under the cables. The additions on sheet 2 relating to the corners do not contain any data defining the beta angle or the travel speed under the laser at these positions. The user may choose to define the angles at these points but is required to fill in the travel speed data at these points. The additions on sheet 2 relating to the cables do contain data defining the beta angle but not the travel speed under the laser at these positions. The user may choose to edit the angles at these points but is required to fill in the travel speed data at these points. Once the editing is finished to sheet 2 , the user makes a mouse click on the button labeled "Create Full Motion Control Data on Sheet3." This runs the macros that delete the old sheet 3 and create a new one.

\section{Summary of Definition From User on the Second Sheet:}

- Travel speeds are required (the macro will stop with an error if all definition rows do not contain travel speed data).

- Angle changes if desired for corners and under cable moves.

\section{Sheet 2 Definition Notes:}

1. If the cable is close to the start or end of a corner rotation, the user may want the motion to begin or finish the cable move at an angle desirable for entering or exiting the corner area.

2. The macros that create sheet 2 are automated to prevent the operator from needing to put in the coordinates for the corners and the coordinates at the start and stop of cables. To perform this, the macros require the user to designate the corners correctly on sheet 1 (including using the lowercase letter "c") using the notation $\mathrm{c} 1, \mathrm{c} 2, \mathrm{c3}$, or $\mathrm{c} 4$ where the corner number refers to the quadrant of the body fixed coordinate system (refer to Figure 2). The macros that insert the beginning and ending sx' and sy' coordinates for the radiused corners on sheet 2 will not include a beta angle in the angle field. It is up to the user to determine at what point the rotation around a corner should start and end. This allows the user to start a corner rotation early, before encountering the radius, and end the corner after the radius. To make a smooth rotation around a corner, the user would add points to the sheet 1 data before and after the $\mathrm{cl}$, $\mathrm{c} 2, \mathrm{c} 3$, or $\mathrm{c} 4$ designator for the corner. The first point (before the upcoming corner 


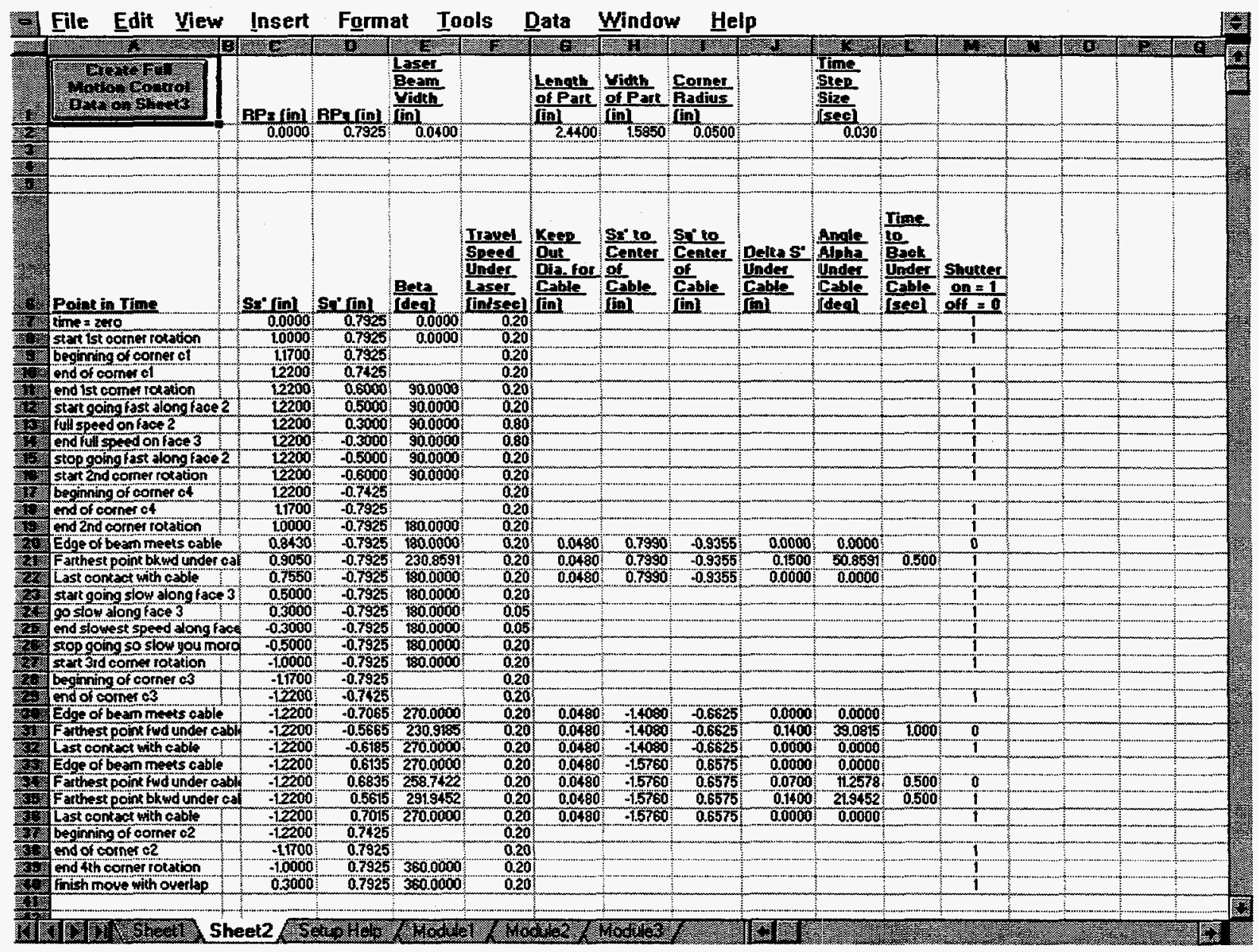

\section{Figure 6. Sheet 2 of Spreadsheet}

designator) would define the $\mathrm{sx}^{\prime}$ and sy' coordinates for the corner rotation to begin. The angle beta for this point would be the current beta angle. Next would be the corner designator. The last point for the corner would be the sx' and sy' coordinates for the corner rotation to end. The angle beta for this point would be the final beta angle for the corner move. The user would then leave the angle fields blank on sheet 2 next to the beginning and ending sx' and sy' coordinates created by the macros for the radiused corners.

3. To define the movement around a cable, the user must define the size of the "keep out diameter" around the cable, the sx' and sy' coordinates to the center of the cable, the distance for the laser to move forward under the cable (if desired), the distance for the laser to move backward under the cable (if desired), and the time in which the shutter turns the laser off while the part is moved and rotated into a position on the other side of the cable. The user may have the laser move both forward and backward under the cable by filling in the distances under the respective columns, but at least one of these distances (forward or backward) must be defined. The macros that create sheet 2 will put in the perpendicular 
angles at the start and stop of a cable move. The user may change these angles on sheet 2 . Because the motion under a cable is well defined, the macros that create sheet 3 correct for an error in the angle definition to keep the laser from shining on the "keep out dia."

4. The user may rotate the part over any interval, but the user is responsible for making sure that the cable is not rotated into the line of sight of the laser during a non-cable move (error checking to keep the laser from shining in the "keep out dia." of the cable only occurs during a cable move).

\section{Sheet 3}

Sheet 3 is a copy of sheet 2 that is expanded to include all of the data calculated to fully define the motion profile and all of the calculations of data needed to create a file for running the motion control system (see Figures 7 and 8).

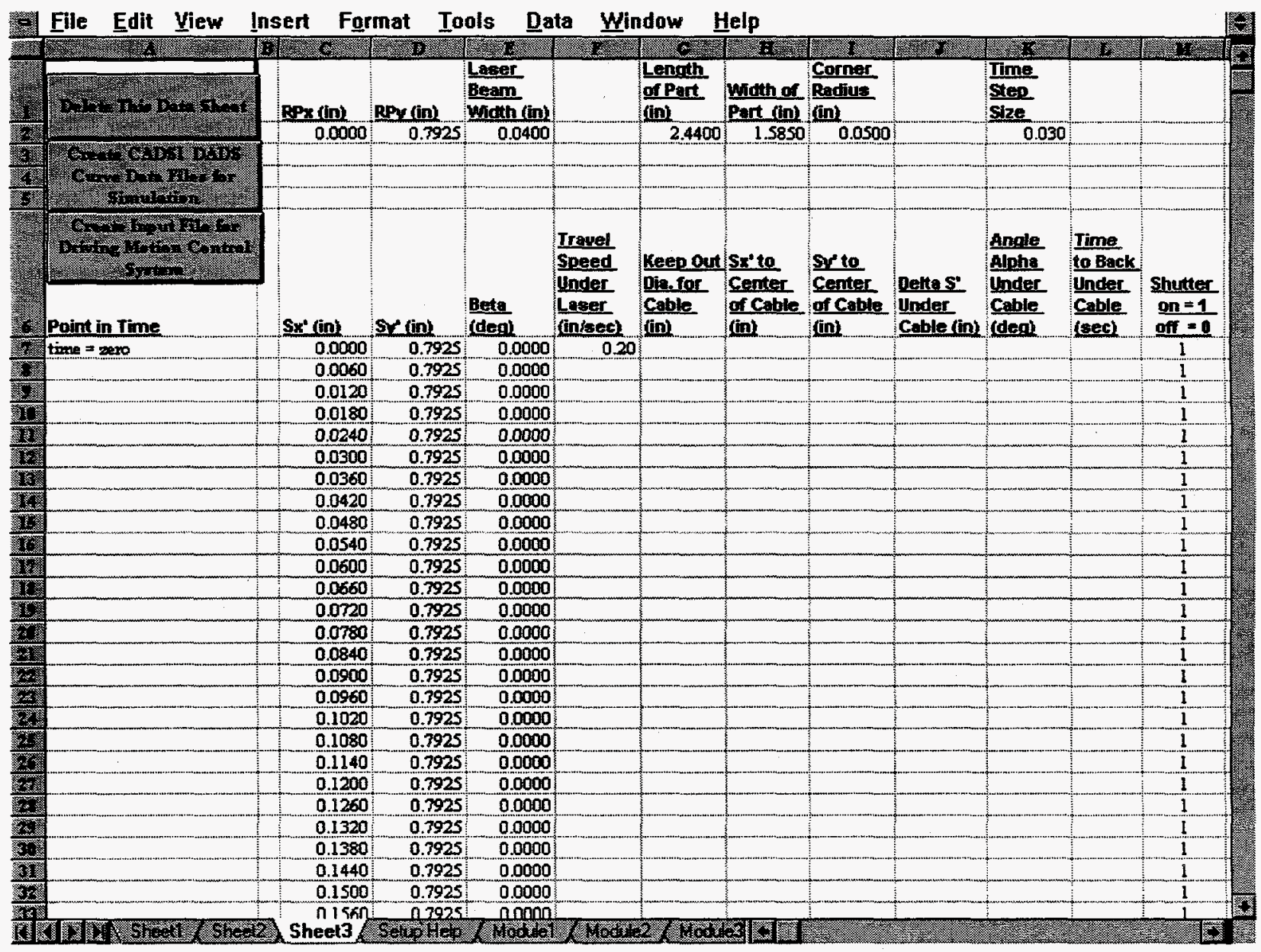

Figure 7. Sheet 3 of Spreadsheet 
The user does not normally edit Sheet 3; it is created entirely when the user clicks on the button on Sheet 2 . Sheet 3 becomes quite large (a typical length of sheet 3 will be about 2000 rows). Sheet 3 contains three buttons. The button labeled "Create Input File for Driving Motion Control System" copies data from sheet 3 into a comma-delimited ASCII file used to drive the motion control system. The button labeled "Create CADSI DADS Curve Data Files for Simulation" generates the ASCII text files called rax.txt, ray.txt, beta.txt, and shutter.txt; these may be used to simulate the motion of the part using the Computer Aided Design Software Inc. (CADSI) DADS dynamic analysis program. The button labeled "Delete This Data Sheet" allows the user to easily delete sheet 3 without altering sheet 1 or sheet 2 . Because sheet 3 is so large and can easily be recreated by a mouse click of the button on Sheet 2 , this button is useful to allow the user to reduce the size of the spreadsheet file (once the input file for the motion control system and the curve files for simulation have been created).

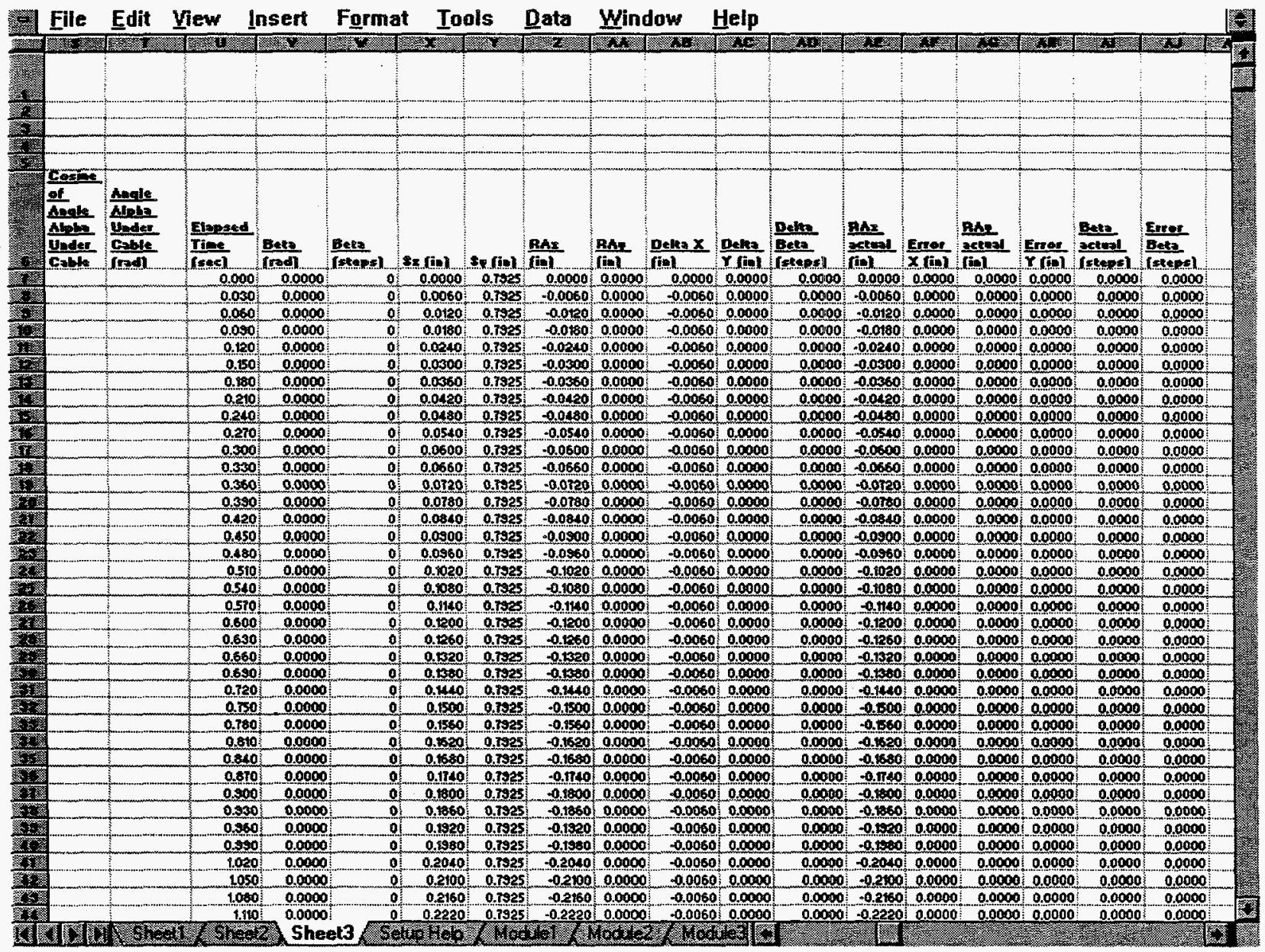

Figure 8. Motion Profile Data Created on Sheet 3 of Spreadsheet 


\section{Sheet Four-Setup Help}

The fourth sheet is labeled "Setup Help" (see Figure 9) and contains a figure and notes to aid the user in defining the motion profile required on sheet 1 .

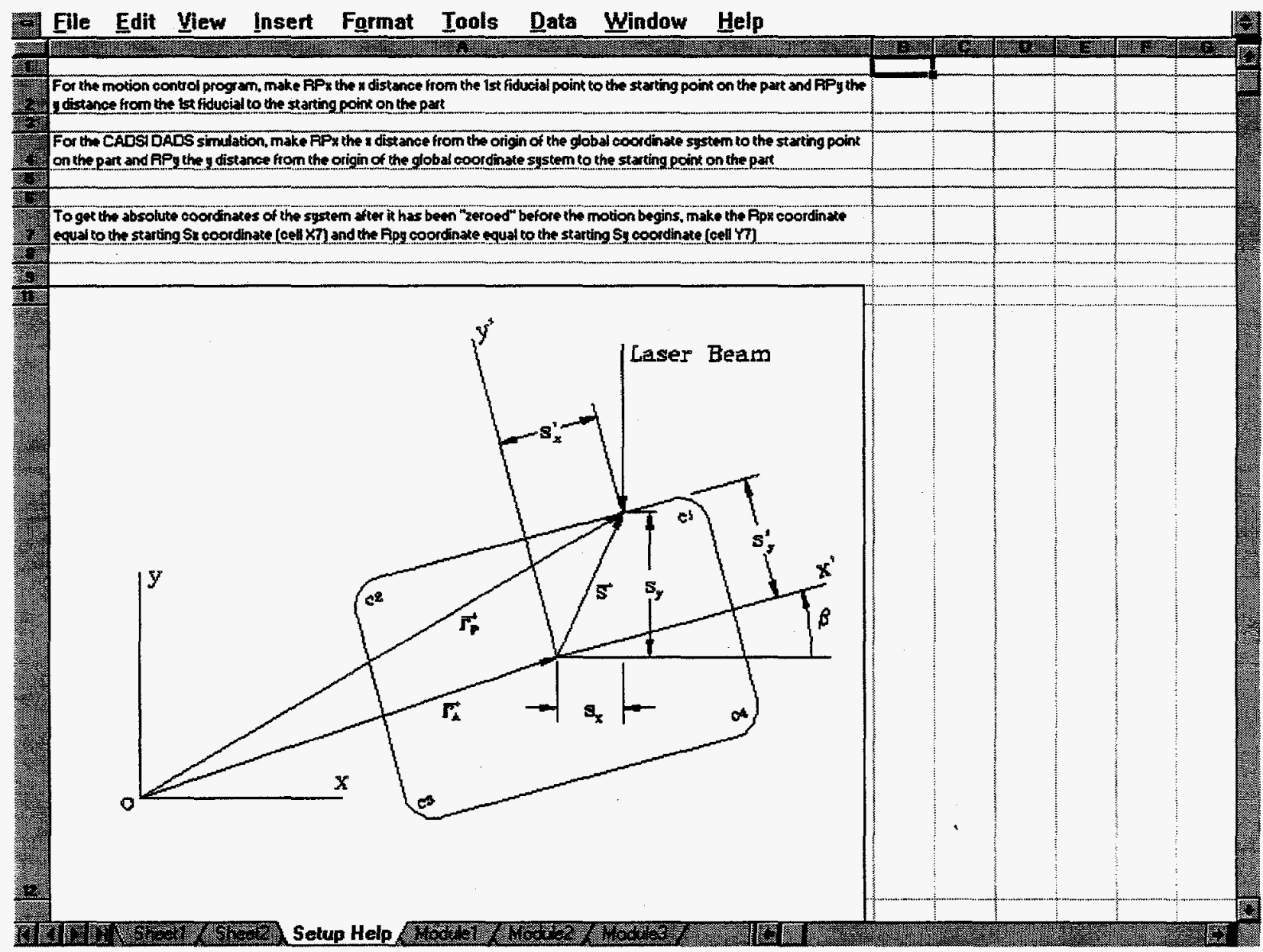

Figure 9. Fourth Sheet of Spreadsheet Labeled "Setup Help"

\section{Simulation of Motion Using DADS}

A verification method was developed by simulating the motion data. The geometry of the assembly was generated using Parametric Technology Corporation Pro/E version 15. This geometry was then converted using Pro/DADS version 3.1 from Computer Aided Design Software Inc. (CADSI) and the simulation was carried out using DADS version 8.0 from CADSI. This process animated the results of the motion of the part on the computer screen. This was extremely useful in catching and correcting errors in the Excel spreadsheet (see Figure 10). 


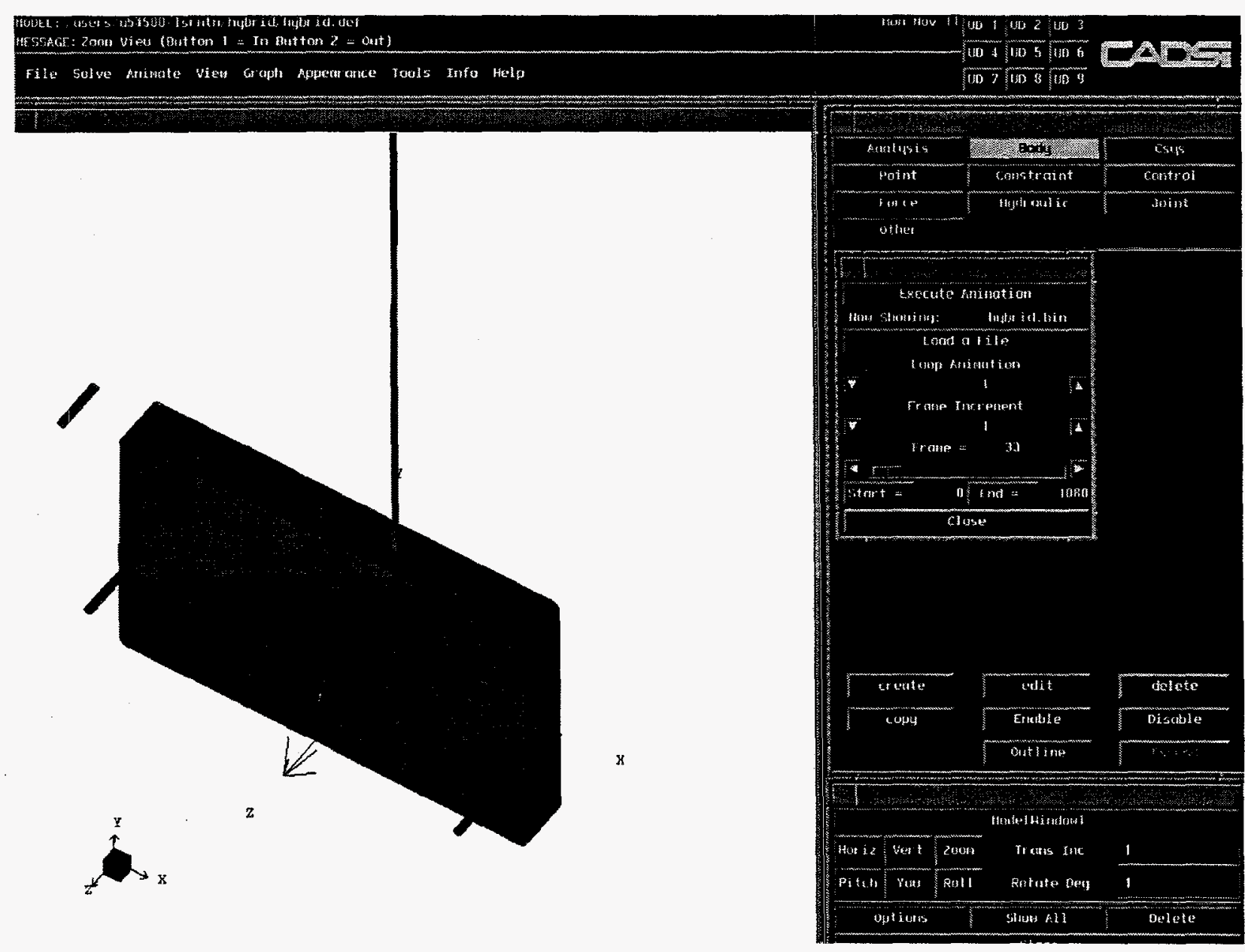

Figure 10. Motion Simulation Using DADS 8.0

\section{Notes on Method Used to Verify Spreadsheet Data}

The following are notes of the method that was used to verify the data from the Excel spreadsheet.

\section{Create Hybrid Geometry in Pro/E:}

1. Create default datums.

2. Create a protrusion from both sides of the sketching plane.

3. Sketch a block shape of part on Datum 3, and when prompted for a horizontal or vertical reference for sketching, pick Top and then Datum 2. 
4. Create vertical and horizontal centerlines and align them to Datum 1 and Datum 2.

5. Draw a rectangle centered around the centerlines, and dimension the length along the $\mathrm{x}$ direction and the width along the $\mathrm{y}$.

6. Radius the corners by creating a round and selecting the edge of each corner.

7. Create a separate circular protrusion for each cable, pick Both Sides and again sketch on Datum 3 with Top as Datum 2. Sketch a circle and dimension the diameter and the center of the circle from the Datums 1 and 2.

\section{Create Laser Beam Geometry in Pro:}

1. Create default datums.

2. Create a protrusion from One Side of a sketching plane.

3. Sketch on Datum 2 with feature creation up, and when prompted for a horizontal or vertical reference for sketching, pick Bottom and then Datum 3.

4. Sketch a circle with the diameter of the laser beam and the center aligned with Datum 3 and Datum 1.

\section{Create Assembly of Hybrid and Laser Beam and Export Geometry Using Pro/DADS:}

1. Assemble the laser beam to the hybrid. It doesn't matter how the parts are assembled together (the important thing is to include the hybrid and laser parts into an assembly together). No matter how the parts were assembled, they will both come into DADS with their datum planes aligned to the global coordinate system in DADS.

2. Save the assembly file.

3. From the Assembly choose DADS, then File, then Write geo, then All parts. Type the highest number allowable for the quality of the geometry on the Pro/E command line.

4. Now exit without saving (the part geometry slp files have already been saved to disk).

\section{Start DADS and Import the Geometry Files:}

1. Start DADS and choose a two-dimensional analysis.

2. Pick File, Save_as, and name the project definition file hybrid.

3. Pick File, Import, Geometry, and then slp. Go to the directory with the .slp file for the hybrid and select it. Repeat for the .slp file for the laser.

4. Create a Body for the Hybrid and Assign to it the Hybrid Geometry:

5. Pick Body and then Create. 
6. Highlight the part name and change the name to hybrid.

7. Pick Use on the Geometry row, Use GeomShell, and pick hybrid.

8. Change from Wireframe to Solid (if desired) and change colors using slide bars next to red, green, and blue colors (if desired).

9. There are three labels and triads attached to the part. To turn them off, select the Body submenu, and toggle the "Enable" to "Disable" next to the wording labeled "Show Triad \& Label." The first selection is located under Body, the next is under Center of Gravity, and the last selection is located by picking Rotate/Translate under "Geometry" Edit.

10. Choose Accept.

\section{Create a Body for the Laser and Assign to it the Laser Geometry:}

1. Pick Body and then Create.

2. Change the name to laser.

3. Pick Use on the Geometry row, Use GeomShell, and pick laser.

4. Change from Wireframe to Solid (if desired) and change colors using slide bars next to red, green, and blue colors (if desired).

5. Translate the laser beam by picking Rotate/Trans and type in a value equal to $1 / 2$ the width of the part for the $y$ coordinate (coordinates are in the format $x, y, z$ ). This will put the end of the laser beam on the top edge of the hybrid.

6. There are three labels and triads attached to the part. To turn them off, select the Body submenu, and toggle the "Enable" to "Disable" next to the wording labeled "Show Triad \& Label." The first selection is located under Body, the next is under Center of Gravity, and the last selection is located by picking Rotate/Translate under "Geometry" Edit.

\section{Creating Curves and Drivers for Defining the Motion of the Hybrid and Laser Beam}

The purpose of the next steps is to satisfy the definition of the three degrees of freedom (of a two-dimensional analysis) for both the hybrid and laser. To satisfy the definition requirements, the $\mathrm{x}, \mathrm{y}$, and angle degrees of freedom will be defined as a function of time for both the hybrid and laser. The hybrid will move in the $x, y$ directions and rotate. The laser will not move in the $x$ direction or rotate, but to animate the effect of shuttering off the laser, it will be almost instantaneously "jerked" out of the field of view in the y direction whenever the laser is shuttered off.

The definition of the movements of the hybrid and laser is accomplished in DADS by first creating curves consisting of time versus position data, and then creating drivers that will use the 
curves to move the part according to the specified curve. To create the curves, two lines of data will be typed in. This data corresponds to a position of zero at time zero and also a position of zero at time one second. This data is all that is needed for the curves used by the drivers that keep the laser from moving in the $\mathrm{x}$ direction and rotation. For the rest of the movements needed, this data will be edited by using a text editor to paste in the Excel file data (rax.txt, ray.txt, beta.txt, and shutter.txt) on top of it.

\section{Create Curves to Drive Hybrid and Laser:}

1. From the DADS Modeling dialog box, pick Other, Curve, SplineCurve, Create.

2. Rename the curve to rax.

3. Make sure the Type of Data is Spline.Explicit.

4. Click on Interpolation and pick Linear.

5. Click on DATA Edit and then click in the editor window. Make an array of numbers such as follows and then click Done.

$0 \quad 0$

10

6. Accept the Curve form.

7. Click copy, make a copy of the previously defined curve, and then edit the new curve, changing its name to ray.

8. Make another copy of a previously defined curve, and then edit the new curve, changing its name to beta.

9. Make another copy of a previously defined curve, and then edit the new curve, changing its name to Isr_fixed.

10. Make another copy of a previously defined curve, and then edit the new curve, changing its name to shutter.

\section{Create Drivers for the Hybrid:}

1. From the DADS Modeling dialog box, pick Constraint, Driver, One-Body, Create.

2. Rename the Driver to hybrid_x.

3. Toggle the Type to $\mathbf{X}$.

4. Toggle the Body Name to hybrid.

5. Toggle the Triad on Body to either hybrid_cg or hybrid (they are both at the center of the hybrid).

6. Toggle the Function Type to General. 
7. Toggle the Curve Driver to rax.

8. Accept the Driver form.

9. Click copy, make a copy of the hybrid_x driver, and then edit the new Driver, changing its name to hybrid_y, toggle the Type to $\bar{Y}$, and toggle the Curve Driver to ray.

10. Make a copy of one of the previous drivers, and then edit the new Driver, changing its name to hybrid_beta, toggle the Type to PHI, toggle the Curve Driver to beta, and toggle the Angular Units to Radians.

\section{Create Drivers for the Laser:}

1. From the DADS Modeling dialog box, pick Constraint, Driver, One-Body, Create.

2. Rename the Driver to laser_ $\mathbf{x}$.

3. Toggle the Type to $\mathbf{X}$.

4. Toggle the Body Name to laser.

5. Toggle the Triad on Body to either laser_cg or laser (they are both at the center of the laser).

6. Toggle the Function Type to General.

7. Toggle the Curve Driver to Isr_fixed.

8. Accept the Driver form.

9. Make a copy of the laser_ $\mathbf{x}$ driver, and then edit the last Driver, changing its name to laser_y, toggle the Type to $Y$, and toggle the Curve Driver to shutter.

10. Make a copy of the laser_ $\mathbf{x}$ driver, and then edit the new Driver, changing its name to laser_beta, toggle the type to PHI, and make sure the Curve Driver is Isr_fixed.

\section{Save and Then Edit the .def File:}

1. Pick File and Save to save the project definition file hybrid.def.

2. Using the FTP utility, copy the ASCII files rax.txt, ray.txt, beta.txt, and shutter.txt from the directory containing the Excel 5.0 spreadsheet to the workstation used for the DADS simulation.

3. Using a text editor, copy the data from the rax.txt file (created by the spreadsheet) into the clipboard, and then paste this data on top of the data for the rax curve in the hybrid.def file.

4. Repeat copying and pasting of the ray.txt file to the data for the ray curve, beta.txt to the data for the beta curve, and shutter.txt to the data for the shutter curve in the hybrid.def file.

5. Now the corrected hybrid.def file is the edited version on disk, not the version currently running in memory. To bring the corrected version from disk into the current DADS session, pick File, Open. Pick the hybrid.def file, and then choose Load without Saving (this is 
very important, because if you choose Save Current Project, it will write over the corrected file on the disk).

\section{Run the Analysis and Animate the Results:}

1. Pick Solve from the title bar, toggle the Analysis Type to Kinematic, and set the End Time to slightly greater than the largest elapsed time in the rax, ray, beta, and shutter files.

2. Pick Animate from the title bar, pick Load a File and choose the hybrid.bin file, and then pick Execute Animation.

\section{ACCOMPLISHMENTS}

A method was developed for creating the motion control data for sealing an electronic housing using laser soldering. The motion required to move the housing under the laser is a nonstandard application and was performed with a four-axis system using the timed data streaming mode capabilities of a Compumotor AT6400 indexer. A Microsoft Excel 5.0 spreadsheet was created to calculate the movement of the part under the laser, and macros were written into the spreadsheet to allow the user to easily create this data. A data verification method was developed for simulating the motion data. The method for driving the housing and spreadsheet were verified by simulating the housing movement using DADS version 8.0 from Computer Aided Design Software Inc. (CADSI). 


\section{APPENDIX}

\section{Exel 5.0 Macros to Generate Motion Control Data}


Const first row $=7$

Dim current row As Integer

Dim Ingth $A \bar{s}$ Single

Dim wath As single

Dim radius As Single

Dim beam As Single

Dim time_step As Single

Dim dia As single

Dim ave_speed As Single

Dim sxb_initial As Single

Dim syb-initial As single

Dim sxb_final As Single

Dim syb final As Single

Dim sxcb As Single

Dim sycb As single

Dim time_shutter off As single

Dim half_beam_and_cable As Single

Dim deltās $s \bar{w}$ d Às single

Dim delta_s_bkwd As Single

Dim syb As s̄ingle

Dim sxb As single

Dim cntclock As Integer

Dim direction As Integer

Sub EXPAND_INPUT DATA ()
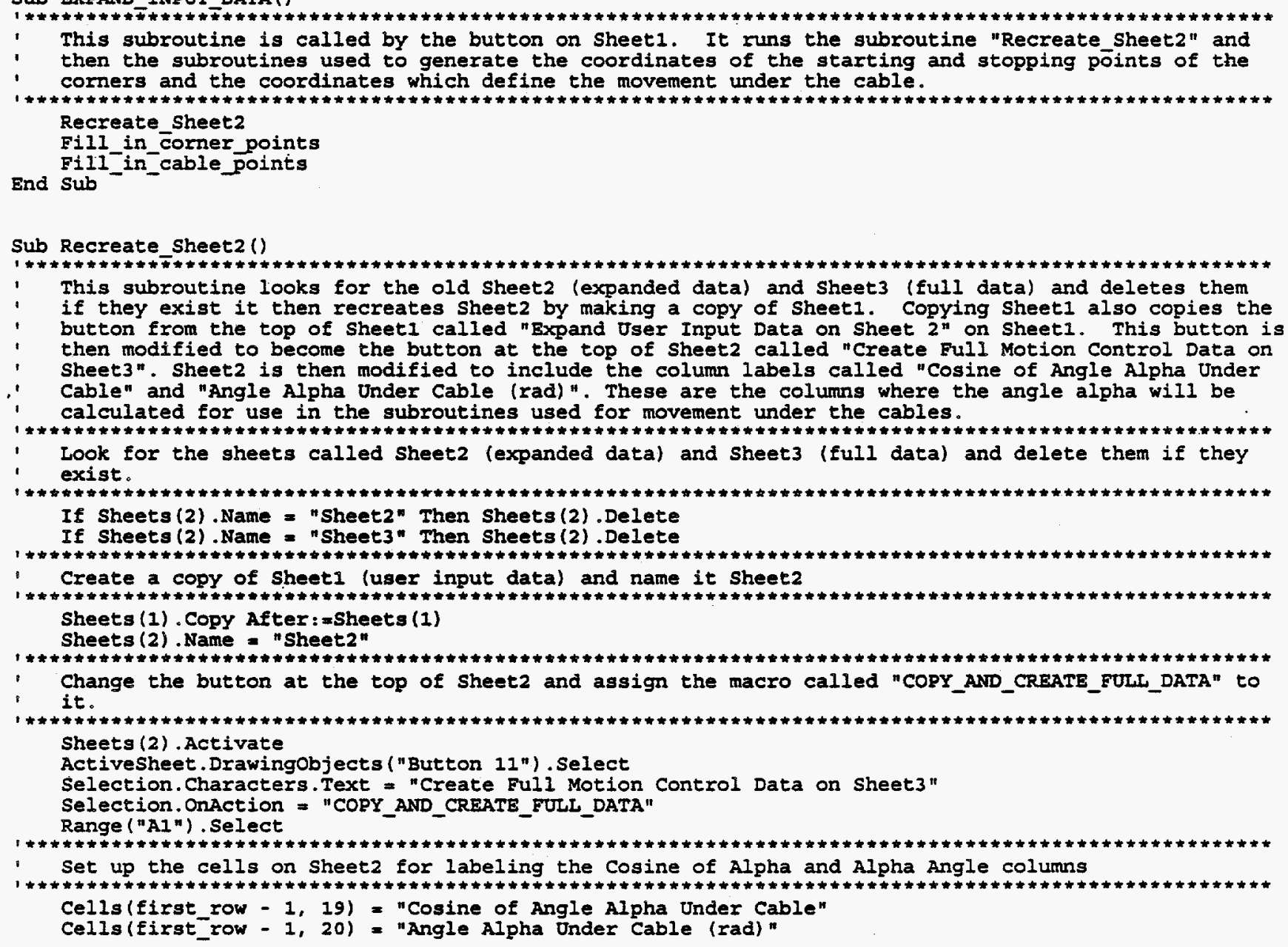
Module1

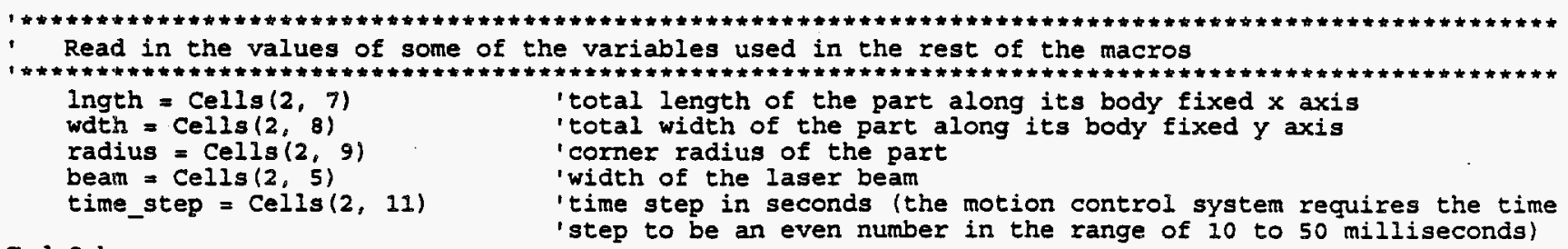

End Sub

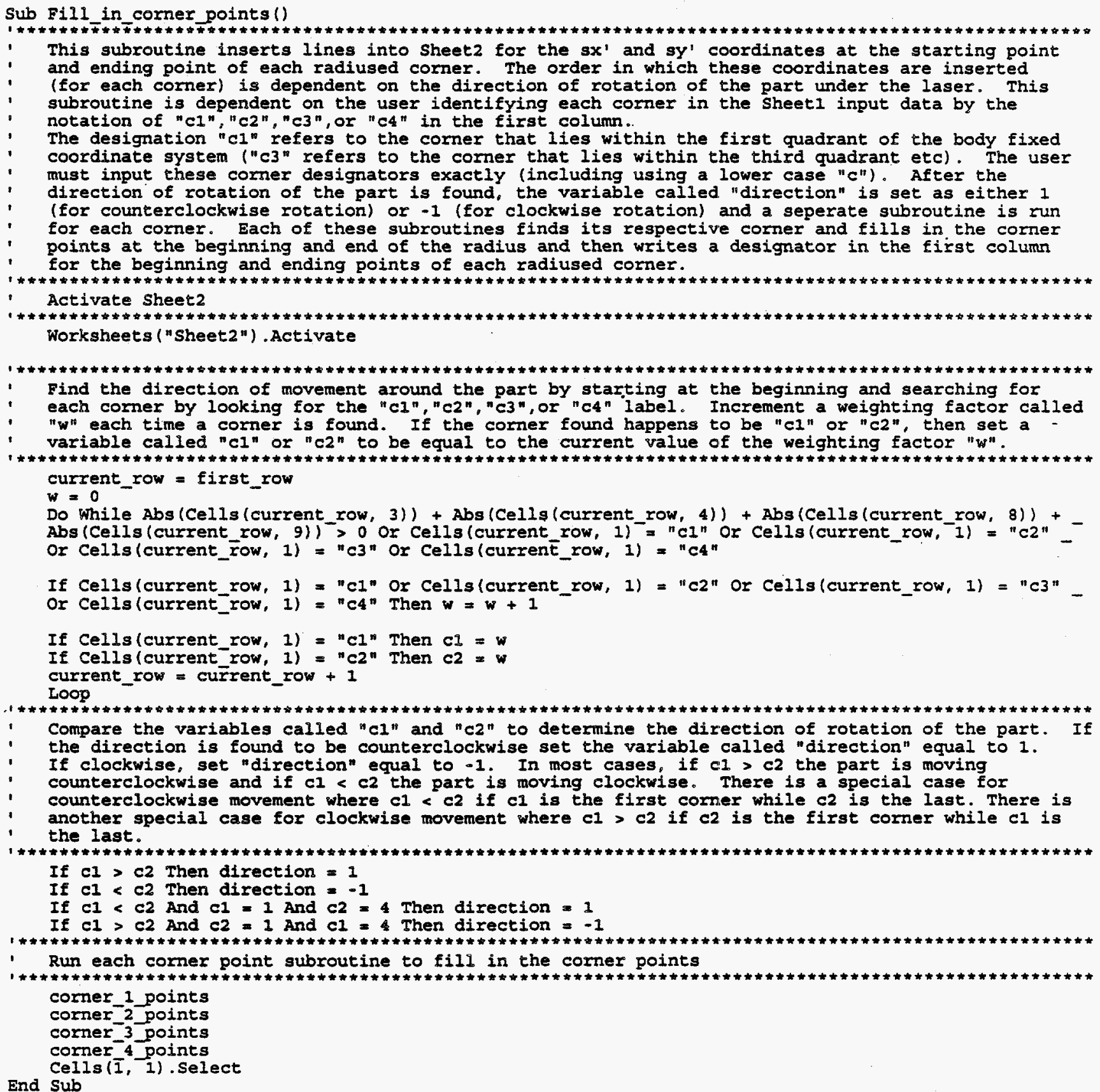


Module1

Sub corner_1 points ()

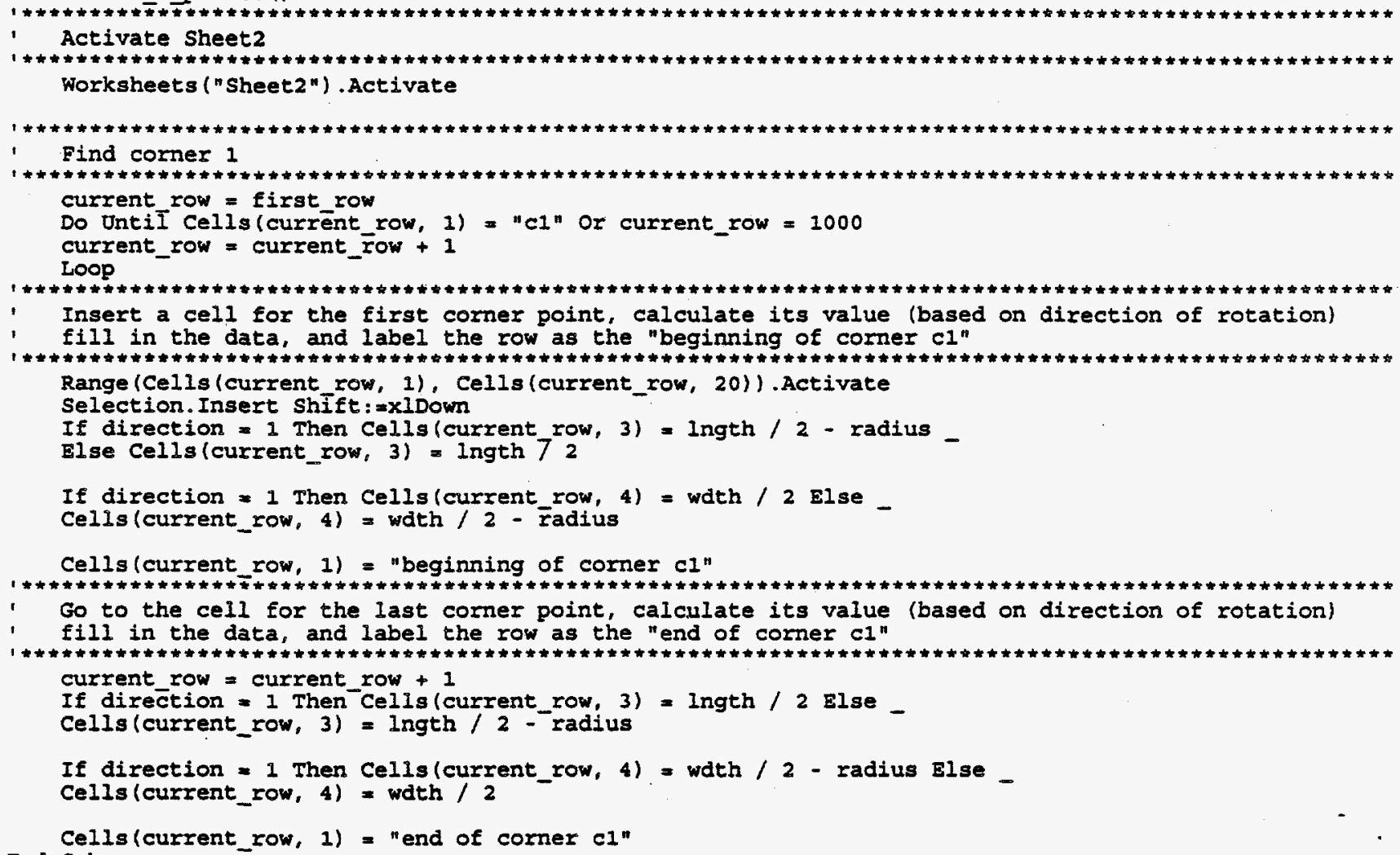

Cells (current_row, 1) = "end of corner c1" End Sub

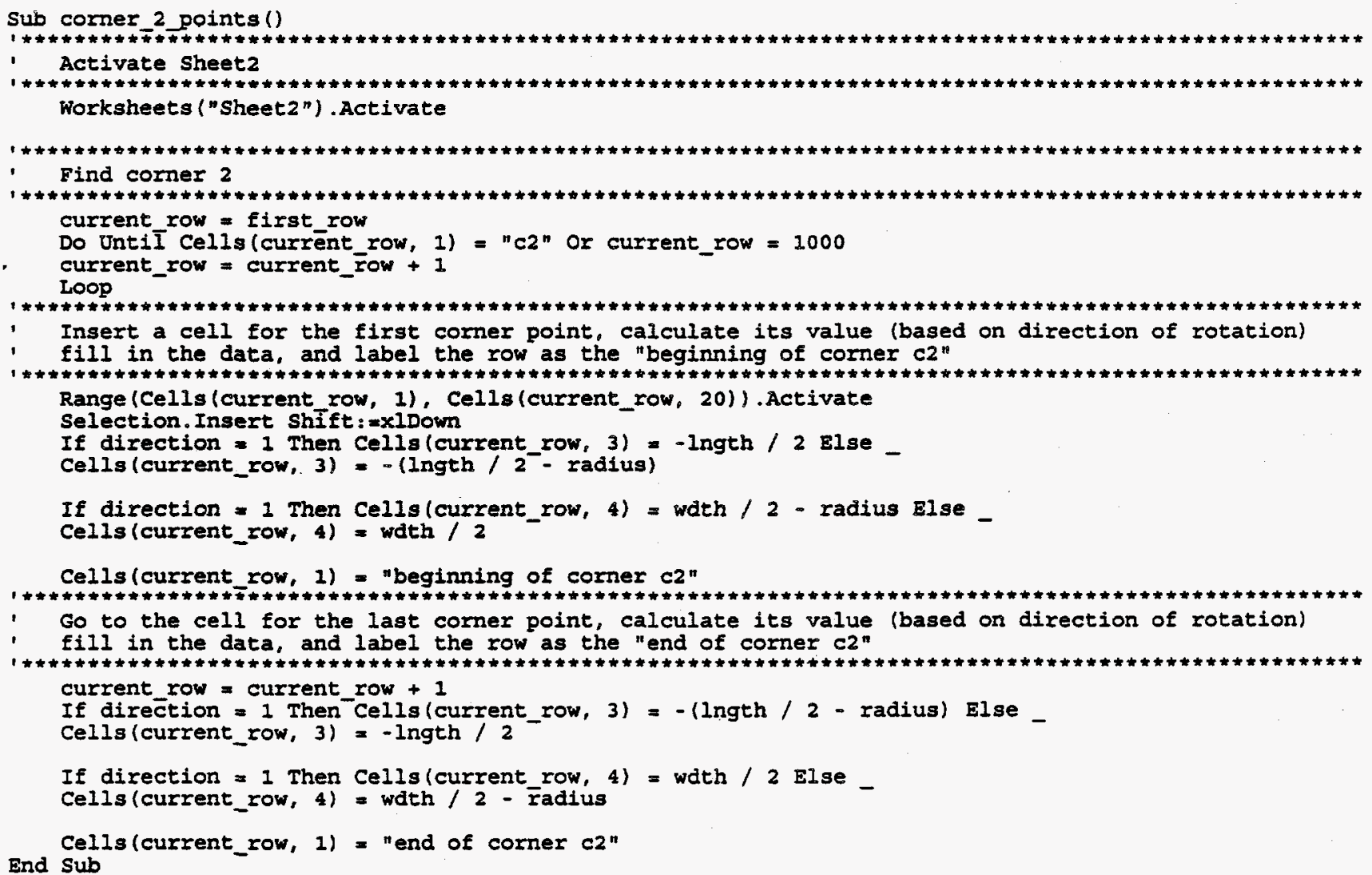




\section{Module1}

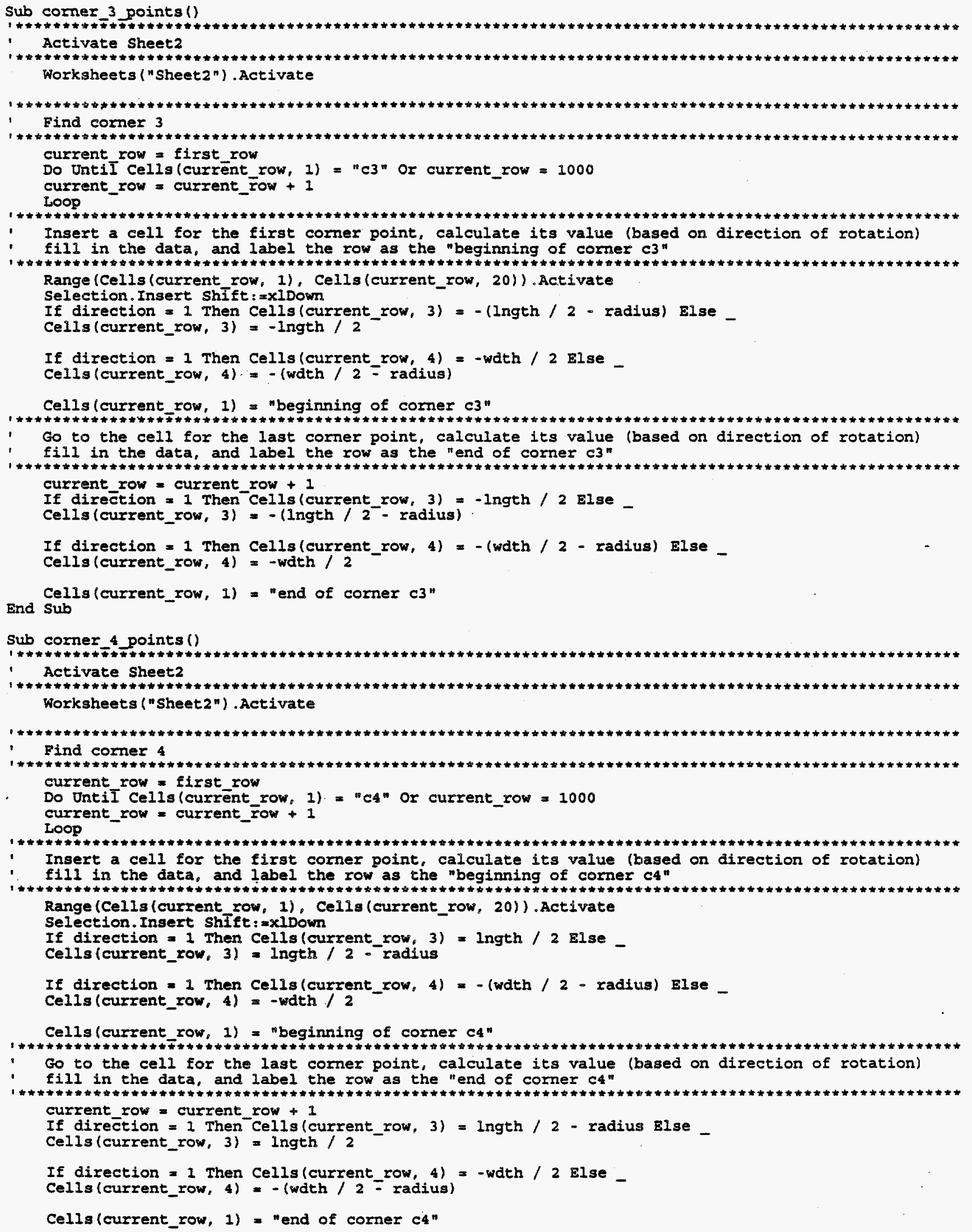


Sub Fill in cable points()

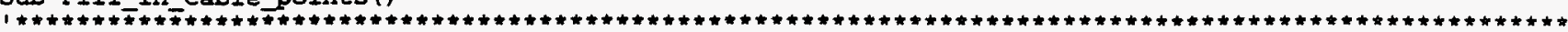
This subroutine inserts lines into sheet2 for the data required for movements around the cables. To define the movement around a cable, the user must define the size of the "keep out diameter" around the cable, the sx' and sy' coordinates to the center of the cable, the distance for the laser to move forward under the cable (if desired), the distance for the laser to move backward under the cable (if desired), and the time in which the shutter turns the laser off while the part is moved and rotated into a position on the other side of the cable. The user may have the laser move both forward and backward under the cable by filling in the distances under the respective columns, but at least one of these distances (forward or backward) must be defined. The subroutine scans down the rows in Sheet 2 looking for a cable definition row (any row which has a value in the "keep out diameter around cable" column). Once a cable definition row is found, the data from that row and the previous row are used to determine which other subroutines will be run for completing the set up definition of the cable move needed on sheet 2 .

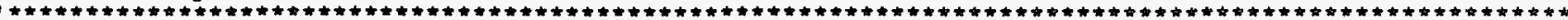
Activate sheet 2

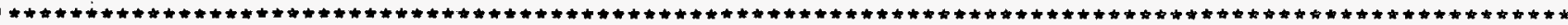
Worksheets ("Sheet2") .Activate

Find the cables (or the end of the data area) by looking for a row which containg data for the "keep out diameter"

i current row $=$ first row +1

Do While Abs (Cells (current row $+1,3))+$ Abs (Cells (current row + 1, 4)) + Abs (Celis (current_row $+1,-8))+$ Abs (Cells (current_row $+1,-9))>0$

If Abs (Cells (current row, 7)) >0 Then cable = True Else cable = False

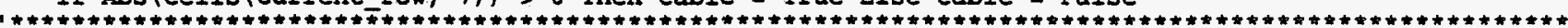
If the current row contains a cable, compare the sx' and sy' coordinates in the previous row to half of the part width or half of the part length to determine if the movement under the cable should be in the $x$ direction or the $y$ direction. Run the macro fill cable $x$ for movement in the $x$ direction and the macro fill_cable_y for movement in the $y$ direction.

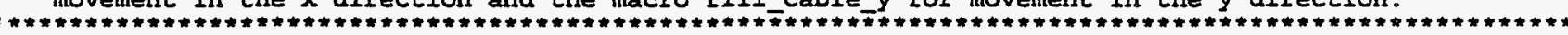
If $\mathrm{cable}=$ True And Abs (Abs (Cells (current row - 1, 4)) - wdth / 2) $<0.00001$ Then fill cable $x$ If cable $=$ True And Abs (Abs (Cells (current_row - 1, 3)) - Ingth $(2)<0.00001$ Then fili_cablē_y current_row $=$ current_row +1

Loop

End Sub

Cells (1, 1). Select

Sub fill cable $x()$

, Worksheets ( "Sheet 2") .Activate

Read in cable definition data from current row. The plus or minus sign of the "delta_s_Ewd" and "delta_s bkwd" values depends on the direction of movement under the cable. This is checked by comparing the coordinates at the start and finish ("sxb initial" and "sxb final" respectively).

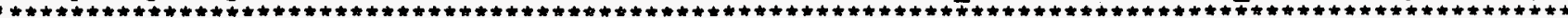
dia $=$ Cells (current_row, 7) keep out diameter around cable

sxb initial = Cells (current row - 1, 3)

syb $=$ Cells (current row - 1,4 )

sxb_final = Cells (current_row $+1,3$ )

sxcb $=$ Cells (current_row, -8 )

sycb $=$ Cells (current row, 9)

'body fixed sx' coordinate from previous row

'body fixed sy' coordinate from previous row

'body fixed sx' coordinate from next row

'body fixed sx' coordinate to the center of the cable 'body fixed sy' coordinate to the center of the cable If sxb_final > sxb_initial Then delta_s_fwd $=$ Cells (current_row, 10) Else delta_s_fwd $=-1 *$ Ceils (current_row, $-1 \overline{0}$ )

If sxb_final > sxb_initial Then delta_s_bkwd = Cells (current_row, 11) Else delta_s_bkwd $=-1$ - Cells (current_row, $\overline{1} 1$ )

time shutter off $=$ Cells (current row, 12 )

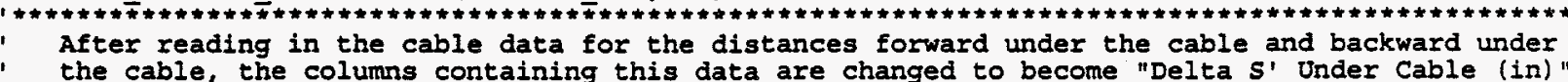
and "Alpha Angle Under Cable (degrees)" respectively.

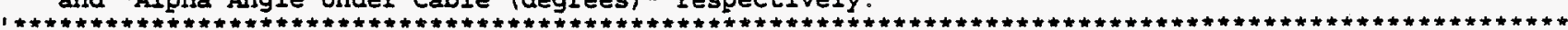
Cells (first row $-1,10$ ) = "Delta s' Under Cable (in)" Cells (firgt row - 1, 11) = "Angle Alpha Under Cable (deg)"

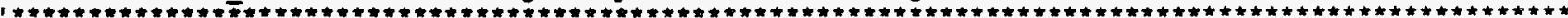
Insert a row, label it "Edge of beam meets cable", calculate the data, and either calculate or just fill in the data for this row. The sx' coordinate at the point where the laser beam edge 
Module 1

meets the cable is found by either adding or subtracting half of the laser beam width and half of the "keep out diameter" from the sx' coordinate of the center of the cable (depending on the direction of travel found by comparing the values of the sx' coordinates in the previous and next rows). The values for the distances "Delta $S^{\prime}$ Under Cable" and "Alpha Angle Under Cable" are zero by default when the laser bean edge first meets the edge of the "keep out area". The previously defined values are filled in for the "keep out diameter", body fixed sx' coordinate to the center of the cable, and body fixed sy' coordinate to the center of the cable. The value for sy' is the same as the value read in for syb. The angle beta is either 0 degrees or 180 degrees at this point (depending on which face the movement occurs). If the face has a positive sy' coordinate, the movement is along the edge defined by sy' $=$ half the width of the part and the beta

angle is 0 degrees. If the face has a negative sy' coordinate, the movement is along the edge defined by $s y^{\prime}=$ negative half the width of the part and the beta angle is 180 degrees.

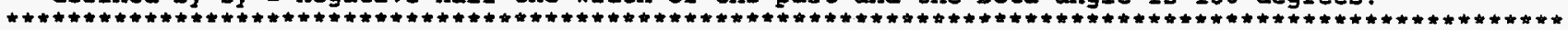
Range (Cells (current row, 1), Cells (current row, 20)). Activate

Selection. Insert Shift: =xlDown

Cells (current_row, 1) " "Edge of beam meets cable"

If sxb_final $>$ sxb_initial Then Cells (current_row, 3 ) = sxcb - (beam + dia) / 2 slse Cells (current row, 3 ) $=$ sxcb $+($ beam $+d i a) / 2$

Cells (current row, 4) $=$ syb

Cells (current row, 7) = dia

Cells (current_row, 8) = sxcb

Celis (current_row, 9) $=$ sycb

Cells (current row, 10) $=0$

Cells (current row, 11) $=0$

If syb $>0$ Then Cells (current_row, 5) $=0$ Else Cells (current_row, 5) $=180$

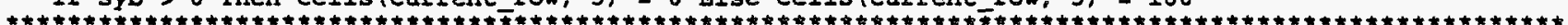

Go to the next row. If the value for moving forward under the cable was non-zero, run the

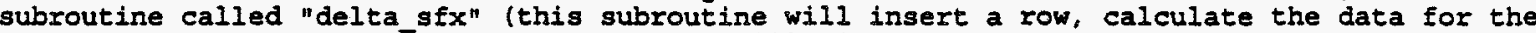
farthest point forward under the cable, and fills in the data for that row). After this, if the value for moving backward under the cable was non-zero, then run the suboutine called "delta sbx" (this subroutine will insert a row, calculate the data for the farthest point backward under the cable, and fill in the data for that row). The value in the shuteer column is always set to zero at the instant before the cable beging to move through the area under the laser beam. If the "delta sfx" subroutine is not run, this point occurs at the edge of the cable and the shutter value is set to zero on the row labeled "Edge of beam meets cable". If the "delta_sfx" subroutine is run, this point occurs at the farthest point forward under the cable and the shutEer value is set to zero on the row inserted by the "delta sfx" subroutine.

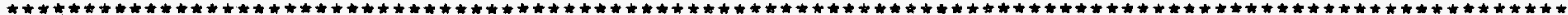
current_row $=$ current_row +1 If Abs(deltas fwd) >- Then delta sfx Else Cells (current_row - 1, 13) = 0 'the else statement 'turns off the shutter if delta_sfx is not run

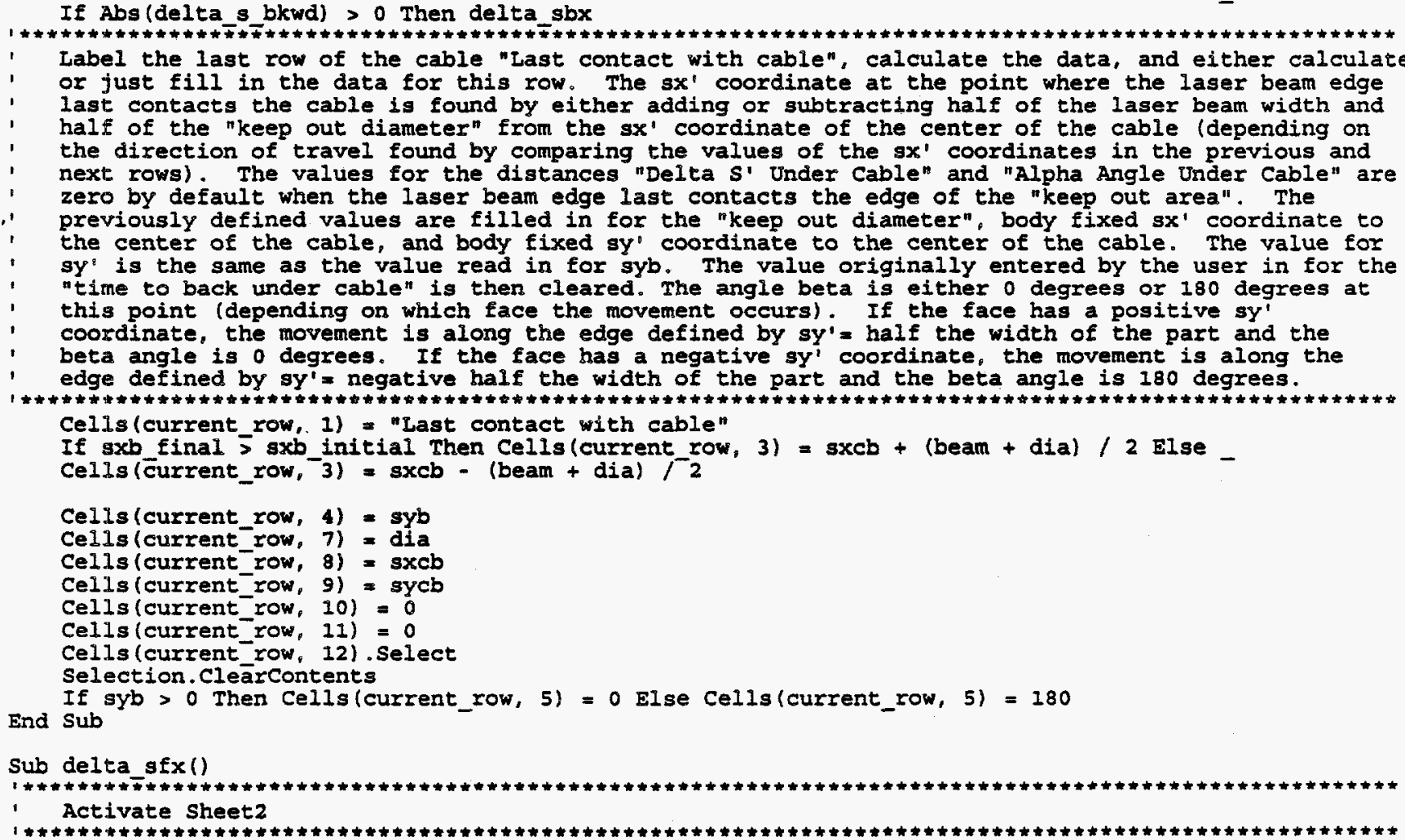
If Abs (delta_s bkwd) >0 Then delta sbx 
Module1

Worksheets ("Sheet2") .Activate

Insert a row, label it "Farthest point fwd under cable", calculate the data, and fill in the data for this row. The sx' coordinate (in column 3) is equal to the sx' coordinate at the center of the cable "sxcb" plus or minus half of the beam and keep out diameter plus or minus the distance forward under the cable (depending on the direction of travel).

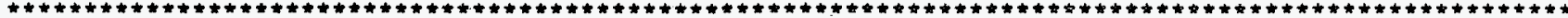
Range (Cells (current_row, 1), Cells (current_row, 20)) .Activate

Selection. Insert Shift: =xlDown

Cells(current row, 1) = "Farthest point fwd under cable"

Cells (current_row, 13) $=0$

If sxb_final $\$$ sxb_initial Then Cells(current_zow, 3 ) $=$ sxcb - (beam + dia) / $2+$ delta_s_fwd Else Cells (current_row, 3 ) $=3 x c b+$ (beam + dia) $/ / 2+$ delta_s_fwd

Cells (current_row, 4) = syb

Cells (current row, 7 ) = dia

Cells (current row, 8) = sxab

Cells (current_row, 9) = sycb

Cells (current_row, 12) = time_shutter_off

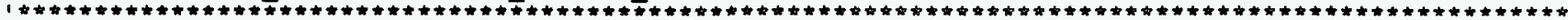

Define the parameters and apply then to the quadratic formula for determining the cosine of the angle alpha under the cable. Write this value for the cosine of alpha into column 19, and then take the arc cosine and write this value as the angle alpha into column 20 . Write the distance "delta $s "$ under the cable in column 10, and convert alpha to degrees and write it into column 11.

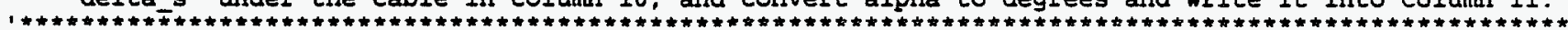
delta $s=$ Abs (delta s fwd)

$\mathrm{H}=\mathrm{A} \overline{\mathrm{b}} \mathbf{s}(\mathrm{sycb}-\mathrm{syb})$

$a=($ delta $s-($ dia + beam $) / 2) \wedge 2+H \wedge 2$

$b=2 \cdot(d \bar{i} a+b e a m) / 2 *($ delta $s+(d i a+b e a m) / 2)$

$c=((d i a+b e a m) / 2) \wedge 2-H \wedge \overline{2}$

cos alpha $=(-b+\operatorname{sqr}(b \wedge 2-4 * a * c)) \cdot /(2 * a)$

Celis (current_row, 19$)=$ cos_alpha

Celis (current row, 20$)="=A \overline{C O S}(R C[-1]) "$

Cells (current row, 10$)=$ deltas

Cells (current_row, 11) $=$ CellsTcurrent_row, 20) $180 \% 3.14159265358979$

i*t: Cells current

Determine the angle beta and write it into column 5 . The angle beta is either 0 or 180 degrees

offset by plus or minus alpha (depending on the edge of the part under the cable, and the direction of rotation of the part. Then increment the value of "current_row", to go on to the next row.

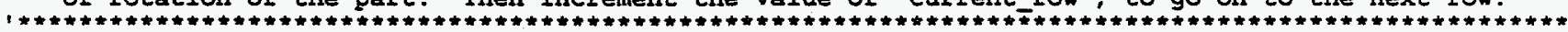

If syb >0 Then Cells (current_row, 5) = 0 - direction - Cells(current_row, 11) Else

Cells (current_row, 5) = 180 - direction * Cells (current_row, 11)

End Sub

current row a current_row +1

Sub delta sbx()

i

Activate Sheet2

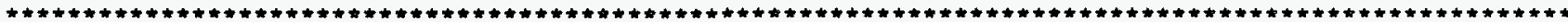

Worksheets ("Sheet2"). Activate

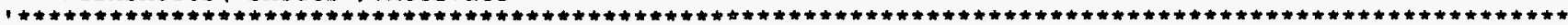

Insert a row, label it "Farthest point blwd under cable", calculate the data, and fill in the data

for this row. The sx' coordinate (in column 3) is equal to the sx' coordinate at the center of the cable "sxcb" plus or minus half of the beam and keep out diameter plus or minus the distance backward under the cable (depending on the direction of travel).

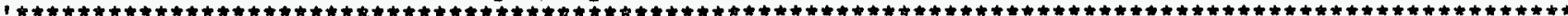

Range (Cells (current_row, 1). Cells (current_fow, 20)). Activate

Selection. Insert Shift: =xlDown

Cells (current row, 1 ) = "Farthest point bkwd under cable"

Cells (current row, 13) = 1

If sxb_final $\bar{s}$ sxb_initial Then Cells(current_row, 3) $=$ sxcb + (beam + dia) / 2 - delta_s_bkwd Else

Cells (current row, 3 ) $=$ sxcb - (beam + dia) $/-2$ - delta s bkwd

Cells (current_row, 4) = syb

Cells (current_row, 7 ) = dia

Cells (current-row, 8 ) $=$ sxcb

Cells (current row, 9) = sycb

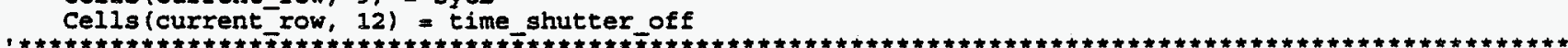

Define the parameters and apply them to the quadratic formula for determining the cosine of the

angle alpha under the cable. Write this value for the cosine of alpha into column 19, and then

take the arc cosine and write this value as the angle alpha into column 20 . Write the distance

"delta_s" under the cable in column 10, and convert alpha to degrees and write it into column 11.

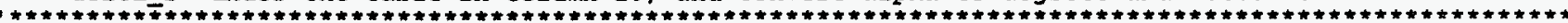

delta_s = Abs (delta_s_bkwd)

$\mathrm{H}=\mathrm{A} \bar{b} \mathbf{s}$ (sycb - syb)

$a=($ delta $\mathbf{s}-($ dia + beam $) / 2$ ) $\wedge+\mathrm{H} \wedge 2$ 
Module1

$b=2 \cdot($ dia + beam) $/ 2 *$ (delta_s $-($ dia + beam $) / 2$ )

$c=((d i a+b e a m) / 2) \wedge 2-H \wedge \overline{2}$

cos alpha $=(-b+\operatorname{Sqr}(b \wedge 2-4 * a * c)) /(2+a)$

CelIs (current_row, 19) = cos_alpha

Cells (current-row, 20) $=$ "ACOS (RC $[-1]$ )"

Cells (current row, 10 ) = delta s

Cells (current_row, 11) $=$ CellsTcurrent_row, 20)*180/3.14159265358979

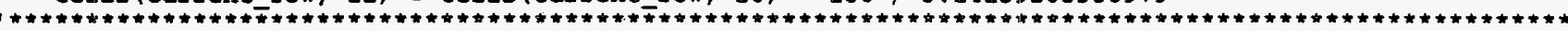

Determine the angle beta and write it into column 5. The angle beta is either 0 or 180 degrees

offset by plus or minus alpha (depending on the edge of the part under the cable, and the direction of rotation of the part. Then increment the value of "current row", to go on to the next row.

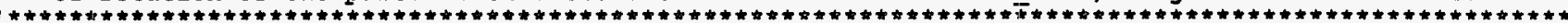

If syb > 0 Then Cells(current_row, 5) $=0+$ direction * Cells(current_row, 11) Else -

Celis (current_row, 5) $=180+$ direction - Cells (current_row, 11)

End Sub

current_row = current_row +1

Sub fill cable $y()$

,

Activate Sheet2

Worksheets ("Sheet 2 "). Activate

Read in cable definition data from current row. The plus or minus sign of the "delta $s$ fwd" and

"delta_s_bkwd" values depends on the direction of movement under the cable. This is checked

by comparing the coordinates at the start and finish ("sybinitial" and "syb_final" respectively).

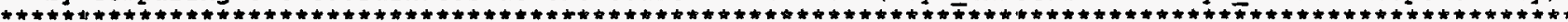

dia $=$ Cells (current_row, 7)

syb_initial $=$ Cells (current_row $-1,4$ )

sxb- = Cells (current row - 1, 3)

syb final = Cells (current row $+1,4$ )

sxcb $=$ Celis (current_row, -8 )

sycb = Cells (current row, 9)

If syb final > syb_iñitial Then delta s fwd = Cells (current_row, 10) Else

delta_s_fwd $=-1$ - Cells (current_row, $1 \overline{0}$ )

If syb final > syb initial Then deltas bkwd = Cells(current_row, 11) Else delta_s_bkwd $=-1 \overline{\bar{s}}$ Cells (current_row, $\overline{11}$ )

time shutter off $=$ Cells (current row, 12)

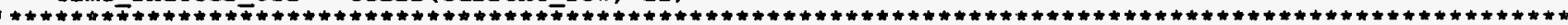

After reading in the cable data for the distances forward under the cable and backward under

the cable, the columns containing this data are changed to become "Delta s' under Cable (in)" and "Alpha Angle Onder Cable (degrees)" respectively.

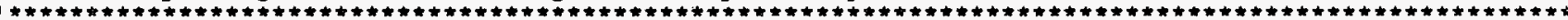

Cells(first_row $-1,10$ ) = "Delta S' Under Cable (ir)"

Cells (first-row - 1, 11) = "Angle Alpha Under Cable (deg)"

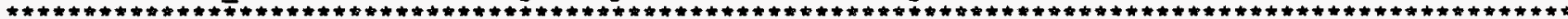

Insert a row, label it "Edge of beam meets cable", calculate the data, and either calculate or

just fill in the data for this row. The sy' coordinate at the point where the laser beam edge

meets the cable is found by either adding or subtracting half of the laser beam width and half of

the "keep out diameter" from the sy' coordinate of the center of the cable (depending on the

direction of travel found by comparing the values of the $3 x^{\circ}$ coordinates in the previous and next

rows). The values for the distances "Delta $S$ ' Under Cable" and "Alpha Angle Under Cable" are zero by default when the laser bean edge first meets the edge of the "keep out area". The previously defined values are filled in for the "keep out diameter", body fixed sx" coordinate to the center of the cable, and body fixed sy' coordinate to the center of the cable. The value for sx' is the same as the value read in for sxb. The angle beta is either 90 degrees or 270 degrees at this point (depending on which face the movement occurs). If the face has a positive sx' coordinate, the movement is along the edge defined by $3 x^{\prime}=$ half the width of the part and the beta angle is 90 degrees. If the face has a negative sx' coordinate, the movement is along the edge defined by $s x^{\prime}$ - negative half the width of the part and the beta angle is 270 degrees.

by $\mathbf{s x}$ : negative half the width of the part and the beta angle is 270 degrees. Range (Cells (current row, 1).Cells (current_row, 20)). Activate

Selection. Insert Shift: $=x$ lDown

Cells (current_row, 1 ) = "gdge of beam meets cable"

If syb_final $>$ syb_initial Then Cells (current_row, 4) $=$ sycb - (beam + dia) / 2 Else

Cells (current row, -4$)=$ sycb $+($ beam + dia) $/ 2$

Cells (current_row, 3) $=\mathbf{s x b}$

Cells (current row, 7 ) = dia

Cells (current row, 8) $=$ sxcb

Cells (current_row, 9) $=$ sycb

Cells (current_row, 10 ) $=0$

Cells (current row. 11 ) $=0$

If $\mathbf{s x b}>0$ Then Cells (current row, 5 ) $=90$ Else Cells (current row, 5) $=270$

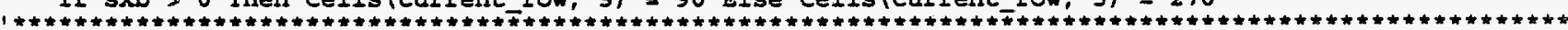

Go to the next row. If the value for moving forward under the cable was non-zero, run the 
subroutine called "delta_sfy" (this subroutine will insert a row, calculate the data for the farthest point forward under the cable, and fills in the data for that row). After this, if the value for moving backward under the cable was non-zero, then run the suboutine called "delta sby" (this subroutine will insert a row, calculate the data for the farthest point backward under the cable, and fills in the data for that row). The value in the shutter column is always set to zero at the ingtant before the cable begins to move through the area under the laser beam. If the "delta sfy" subroutine is not run, this point occurs at the edge of the cable and the shutter value is set to zero on the row labeled "Edge of beam meets cable". If the "delta_sfy" subroutine is run, this point occurs at the farthest point forward under the cable and the shutter value is set to zero on the row inserted by the "delta sfy" subroutine.

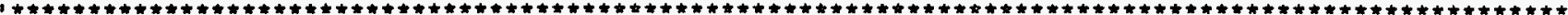
current row $=$ current_row +1

If Abs (delta_s_fwd) >- Then delta_sfy Else Cells (current_row $-1,13$ ) = 0 'the else statement 'turns off the shutter

If Abs (delta_s_bkwd) >0 Then delta sby if delta_sfy is not run

Label the last row of the cable "Last contact with cable", calculate the data, and either

calculate or just fill in the data for this row. The sy' coordinate at the point where the laser beam edge last contacts the cable is found by either adding or subtracting half of the laser beam width and half of the "keep out diameter" from the sy" coordinate of the center of the cable (depending on the direction of travel found by comparing the values of the sy' coordinates in the previous and next rows). The values for the distances "Delta $S^{\prime}$ Under Cable" and "Alpha Angle Under Cable" are zero by default when the laser beam edge last contacts the edge of the

"keep out area". The previously defined values are filled in for the "keep out diameter". body fixed sx' coordinate to the center of the cable, and body fixed sy' coordinate to the center of the cable. The value for $\mathbf{s x}$ ' is the same as the value read in for sxb. The value originally entered by the user in for. the "time to back under cable" is then cleared. The angle beta is either 90 degrees or 270 degrees at this point (depending on which face the movement occurs). If the face has a positive $\mathbf{s x}^{\prime}$ coordinate, the movement is along the edge defined by $\mathbf{s x}$ ' = half the length of the part and the beta angle is 90 degrees. If the face has a negative sx' coordinate, the movement is along the edge defined by $\mathbf{s x}$ ' = negative half the length of the part and the beta angle is 270 degrees.

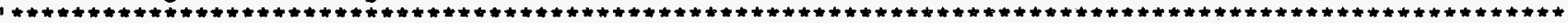

Cells (current row, 1 ) = "Last contact with cable"

If syb_final $>$ syb_initial Then Cells (current_row, 4$)=3 y c b+($ beam + dia) / 2 Else -

Celis (current_row, -4$)=$ sycb - (beam + dia) $/ 2$

Cells (current_row, 3) $=\mathbf{s x b}$

Cells (current_row, 7) $=$ dia

Cells (current row, 8 ) $=$ sxcb

Cells (current_row, 9) = sycb

Cells (current_row, 10) $=0$

Cells (current row, 11) $=0$

Cells (current row, 12). Select

Selection. Clearcontents

End Sub

If sxb $>0$ Then Cells (current_row, 5) $=90$ Else Cells(current_row, 5) $=270$

Sub delta_sfy()

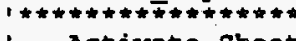

Activate sheet2

Worksheets ("Sheet2"). Activate

(1)

Insert a row, label it "Farthest point fwd under cable", calculate the data, and fill in the data for this row. The sy" coordinate (in column 4) is equal to the sy' coordinate at the center of the cable "sycb" plus or minus half of the beam and keep out diameter plus or minus the distance forward under the cable (depending on the direction of travel).

i t*tot Range (Cells (current row, 1). Cells (current row, 20)) . Activate

Selection. Insert shift: =xldown

Cells (current_row, 1) = "Farthest point fwd under cable"

Cells (current row, 13) $=0$

If syb_final $>$ syb_initial Then Cells (current_row, 4) = sycb - (beam + dia) / $2+$ delta_3_fwd Else Cells (current_row, -4$)=$ sycb + (beam + dia) $/ 72+$ delta_s_fwd

Cells (current row, 3 ) $=$ sxb

Cells (current_row, 7 ) = dia

Cells (current_row, 8) = sxcb

Cells (current row, 9) $=$ sycb

Cells (current row, 12) = time shutter off

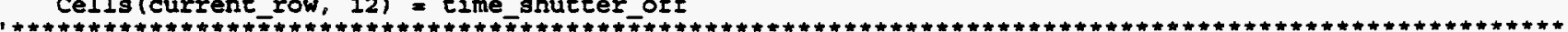

Define the parameters and apply them to the guadratic formula for determining the cosine of the angle alpha under the cable. Write this value for the cosine of alpha into column 19 , and then take the arc cosine and write this value as the angle alpha into column 20 . Write the distance "delta_s" under the cable in column 10, and convert alpha to degrees and write it into column 11.

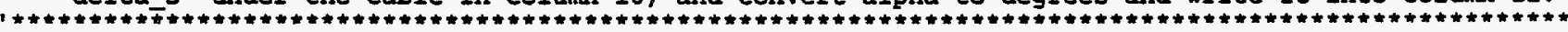
delta_s = Abs (delta_s_fwd) 
$E=A b s(\mathbf{s x c b}-\mathbf{s x b})$

$a=($ deltas $-($ dia + beam $) / 2) \wedge 2+H \wedge 2$

$b=2 \cdot(d \bar{i} a+b e a m) / 2 \cdot($ delta_s $-(d i a+b e a m) / 2)$

$c=(($ dia + beam $) / 2) \wedge 2-H \wedge \overline{2}$

cos alpha $=(-b+\operatorname{Sgr}(b \wedge 2 \cdot 4 * a * c)) /(2 * a)$

Celis (current_row, 19) = cos_alpha

Cells (current row, 20$)=n=A \overline{C O S}(R C[-1]) n$

Cells (current row, 10 ) = delta s

Celis (current_row, 11) $=$ Cells (current_row, 20) $* 180 / 3.14159265358979$

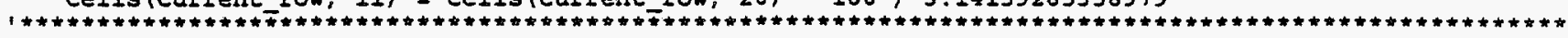

Determine the angle beta and write it into column 5 . The angle beta is either 90 or 270 degrees

offset by plus or minus alpha (depending on the edge of the part under the cable, and the

direction of rotation of the part. Then increment the value of "current_row", to go on to the next row.

1

If $\mathbf{s x b}>0$ Then Cells (current_row, 5) $=90$ - direction - Cells (current_row, 11) Else -

Cells (current_row, 5) 270 - direction * Cells (current_row, 11)

End Sub

current_row $=$ current_row +1

Sub delta_sby()

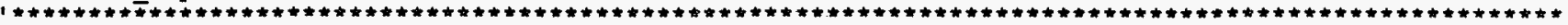

Activate Sheet 2

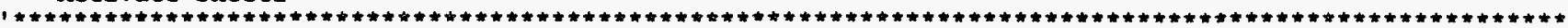

Worksheets ("Sheet $2^{n}$ ). Activate

l

Insert a row, label it "Farthest point bkwd under cable", calculate the data, and fill in the data for this row. The sy' coordinate (in column 4) is equal to the sy' coordinate at the center of

the cable "sycb" plus or minus half of the beam and keep out diameter plus or minus the distance backward under the cable (depending on the direction of travel).

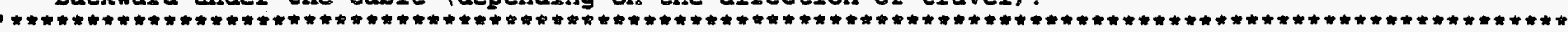

Range (Cells (current row, 1), Cells (current row, 20)) . Activate

Selection. Insert shift: =xlDown

Cells (current_row, 1 ) $=$ "Farthest point bkwd under cable"

Cells (current row, 13) $=1$

If syb_final $>$ syb_initial Then Cells (current_row, 4) $=$ sycb + (beam + dia) / 2 - delta_s_bkwd Else Cells (current_row, 4 ) $=$ sycb - (beam + dia) $/ T_{2} *$ delta_s_bkwd

Cells (current row, 3 ) $=\mathbf{s x b}$

Cells (current_row, 7) = dia

Cells (current row, 8) = sxcb

Cells (current row, 9) = sycb

Cells (current_row, 12) = time_shutter_off

1.* Cellslcurrent-

Define the parameters and apply them to the quadratic formula for determining the cosine of the angle alpha under the cable. Write this value for the cosine of alpha into column 19, and then take the arc cosine and write this value as the angle alpha into column 20 . Write the distance "delta $s^{\prime \prime}$ under the cable in colum 10, and convert alpha to degrees and write it into column 11.

管

delta_s $=$ Abs (delta_s_bkwd)

$\mathrm{H}=\mathrm{A} \bar{b} \mathbf{s}(\mathbf{s} \times \mathrm{cb}-\mathbf{s} \mathbf{x b})$

$a=($ delta $s-($ dia + beam $) / 2) \wedge 2+H^{\wedge} 2$

$b=2 *(d \bar{i} a+b e a m) / 2 *($ delta $s-(d i a+b e a m) / 2)$

$c=((d i a+b e a m) / 2) \wedge 2-H a \overline{2}$

cosalpha $=(-b+\operatorname{sqr}(b * 2-4 \cdot a * c)) /(2 * a)$

Celis (current row, 19) = cos alpha

Cells (current_row, 20) $=$ "=ACOS (RC [-1])"

Cells (current_row, 10$)=$ delta_s

Cells (current row, 11) $=$ CellsTcurrent row, 20) $* 180 / 3.14159265358979$

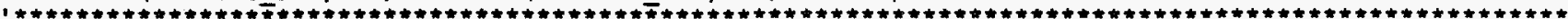

Determine the angle beta and write it into column 5. The angle beta is either 90 or 270 degrees

offset by plus or minus alpha (depending on the edge of the part under the cable, and the

direction of rotation of the part. Then increment the value of "current_row", to go on to the next row.

1

If $\mathrm{sxb}>0$ Then Cells (current_row, 5) $=90+$ direction Cells(current_row, 11) Else

Cells (current_row, 5) $=270+$ direction * Cells (current_row, 11)

End Sub

current row $=$ current row +1 


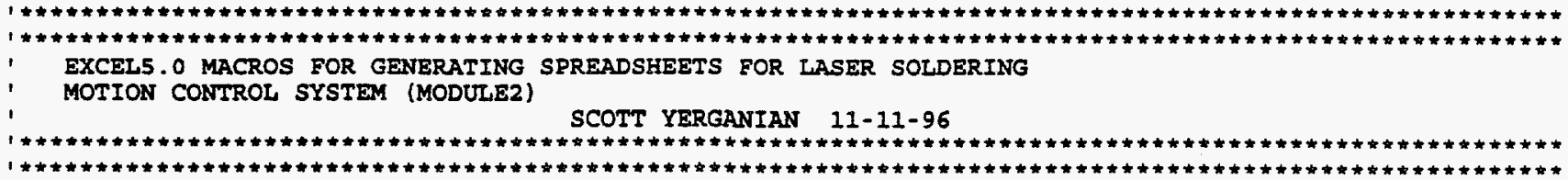

Const first_row $=7$

Dim current row As Integer

Dim Ingth As single

Dim wdth As single

Dim radius As single

Dim beam As single

Dim time step As Single

Dim dia $\bar{A} s$ single

Dim ave speed As Single

Dim sxb-initial As single

Dim syb-initial As single

Dim sxb-final As single

Dim syb final As Single

Dim sxcb As Single

Dim sycb As single

Dim time shutter off As Single

Dim half-beam_and_cable As single

Dim delta_s_fw̃d As single

Dim delta s bkwd As single

Din syb As single

Dim sxb As single

Dim cntclock As Integer

Dim direction As Integer

SUb COPY AND CREATE FULL DATA ()

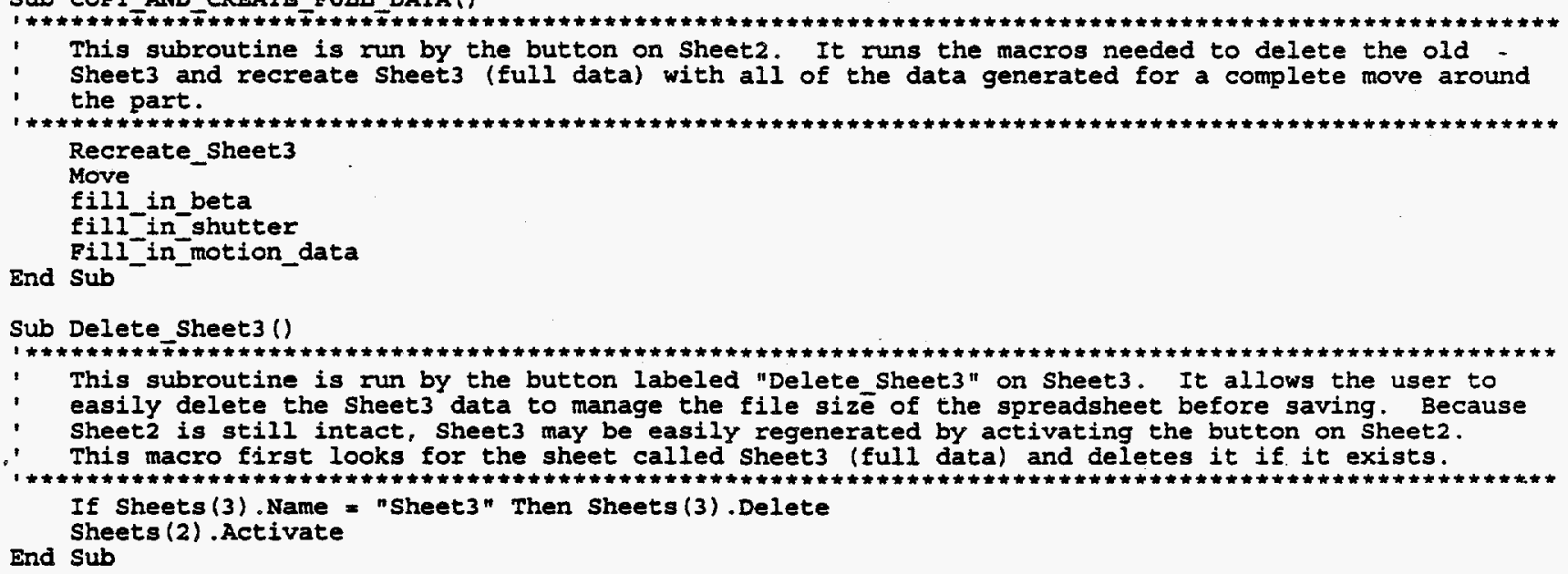

Sub Recreate_Sheet 3 ()

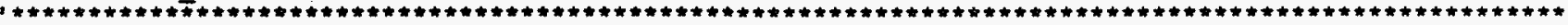
This subroutine looks for the sheet called sheet3 (full data) and deletes it if it exists, then creates a

copy of sheet2 (expanded user input data) and names it sheet3. It then reads in some data needed for the rest of the macros, and runs the macros to create the rest of the buttons at the top of Sheet 3

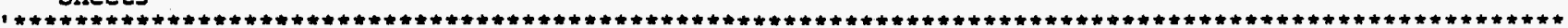
If Sheets (3). Name $=$ "Sheet $3 "$ Then Sheets (3). Delete

Sheets (2) . Copy After: =Sheets (2)

sheets (3). Name = "Sheet3" Range ("AI"). Select

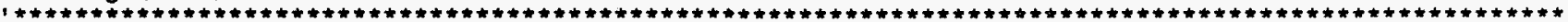
Delete the button from sheet 3

Activesheet.Drawingobjects ("Button 11"). Select Selection. Delete

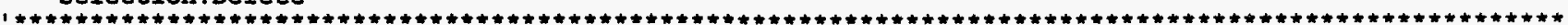

Read in the values of some of the parameters used in the rest of the macros and run the macro to

- create the rest of the buttons at the top of sheet3 
Module2

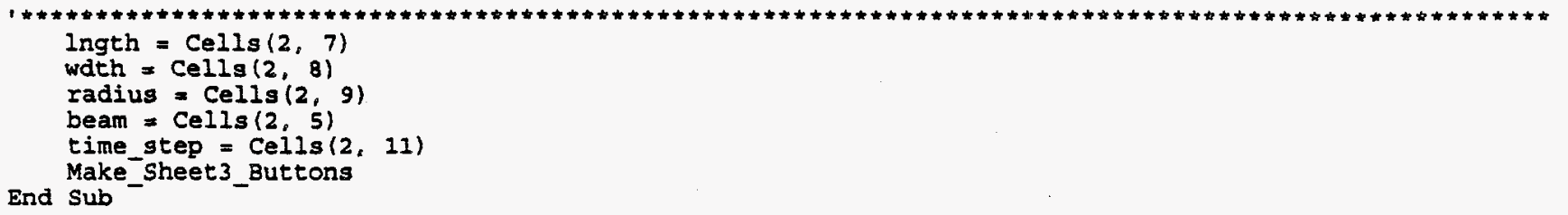

Sub Move ()

†

This macro scans down each row of data copied from sheet2 to sheet 3 . Based on the values of the sx' and sy' coordinates, it chooses which movement macro is required and then runs the macros needed to generate the data describing the complete movement around the part.

† Activate sheet 3

1 Worksheets ("Sheet 3 ") .Activate

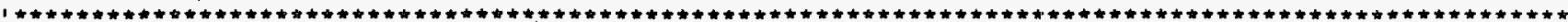
Read in the values of some of the parameters used in the rest of the macros.

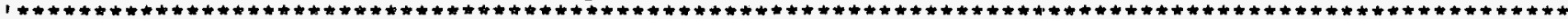

lngth $=\operatorname{Cells}(2,7)$

wdth $=\operatorname{Cells}(2,8)$

radius $=$ Cells $(2,9)$

beam $=\operatorname{Cells}(2,5)$

time_step $=\operatorname{Celis}(2,11)$

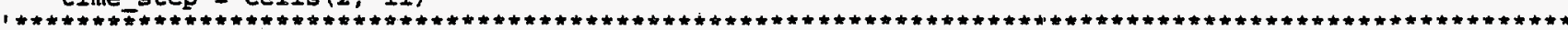

step through each row beginning with the row following "first row", keeping track of current row

position using the variable called "current_row". Set variabies called "move_x", "move_y", or "corner" to be true or false by comparing the sx' and.sy' coordinates (columns ' 3 , and 4) of' the current row, to the part geometry, or if the label in column 1 designates the end of $a$ corner.

In some cases, (at the beginning or ending of a corner move) both "corner" and "move $x$ " or "move $y$ " may be true. The selection logic used later will determine the correct macros in these cases.

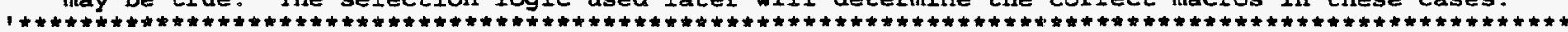
current row $=$ first row +1

Do While Abs (Cells (Current_row, 3)) + Abs (Cells (current_row, 4))>0.00001

If Abs (Abs (Cells (current_row, 4)) - wdth $/ 2$ ) $<0.00001$ Then move_ $x$ = True Else move $x=$ False

If $\mathrm{Abs}$ (Abs (Cells (current row, 3)) - Ingth $/ 2$ ) < 0.00001 Then movē $y=$ True Else move $y=$ False If Abs (Cells (current row, 3)) > Ingth / 2 - radius +0.00001 And

Abs (Cells (current_row, 4i) > wdth /2 - radius +0.00001 Then corner = True Else corner = False

If Cells (current row, 1) $=$ "end of corner c1" Or Cells (current row, 1) = "end of corner c2" Or Cells(current_row, 1 ) $=$ "end of corner c3" Or Cells(current_row, 1 ) = "end of corner c4" Then corner = True

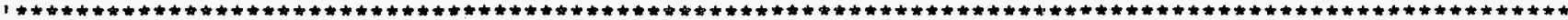
Run the one macro for the movement needed, based on the values of the variables "move $X "$, "move $Y$ ", and "corner". If all of the variables are false, then something is wrong, skip to the end of the subroutine called "last line". Run the macro "Move delta $x$ " "Nove delta $y$ " or "Move corner" and then 10op back to run though the subroutine again. "If the criteria-for both "comer" and "move $x$ " or "move $y$ " are true, then it is actually a corner, and the "Move corner" subroutine is run.

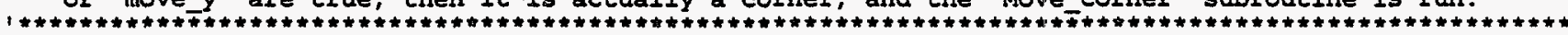

If move $x=$ False And move $y=$ False And corner $=$ False Then GoTo last line

If move $-x=$ True And corne $\bar{x}=$ False Then Move_delta_ $x$

If move $y=$ True And corner = False Then Move_delta_y

If corner $=$ True Then Move comer

Loop

last line:

End Sub

Cells (1, 1). Select

\author{
Sub Move delta $\times()$ \\ 1 \\ Activate sheet 3

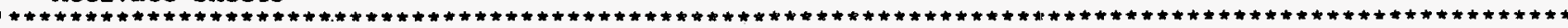 \\ Worksheets ("Sheet 3 ") .Activate \\ Read in the values for the initial gxl coordinate value and the final sx' coordinate value in the \\ interval. Calculate the total distance to move. Compare the values for the initial and final sx' \\ coordinates to determine if the velocity is in the positive or negative directions. Then set the
}


initial and final velocities equal to either plus or minus the travel speed (column 6) values.

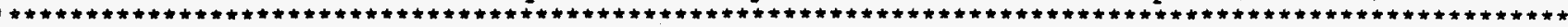
s_initial $=$ Cells (current_row $-1,3$ )

s final $=$ Cells (current row, 3 )

total $s=s$ final - s iñitial

If s_final $>$ s_initiaI Then initial_vel = Cells (current_row - 1, 6) Else _

initial_vel $=-1$ * Cells (current_row $-1,6$ )

If s_final > g_initial Then Einal_vel = Cells (current_row, 6) Else _

finaI_vel $=-1^{-}$Cells (current_row, 6 )

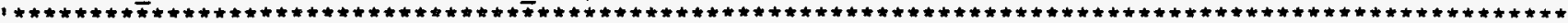

Determine if this is the special case for a quick movement around the cable with the shutter

turned off by checking for the labels designating the farthest points forward or backward under the cable have been reached. If so, the velocity of movement is not governed by the values entered for the travel speed (column 6). In these cases, the travel speed value is used to determine the final velocity of the previous movement. Both the initial and final velocities while moving with the shutter off are set to the value calculated by dividing the distance to travel during this move by the value of the "Time to back under cable" (column 12).

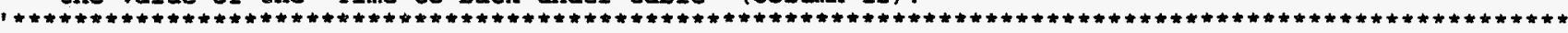
If Cells (current_row, 1) = "Farthest point bkwd under cable" Then initial_vel $=$ (s_final - s_initial)/Cells (current_row, 12)

If Cells (current_row, 1) = "Farthest point bkwd under cable" Then final_vel = initial_vel If Cells (current_row - 1, 1) = "Farthest point fwd under cable" Then initial_vel $=$ (s_final : s_initial) / Cells (current_row - 1, 12)

If Cells (current_row $-1,1)=$ "Farthest point fwd under cable" Then final_vel = initial_vel

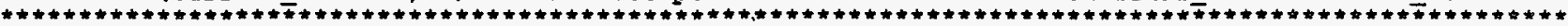
Because the velocity can change over an interval, the equations from physics for a body moving under constant acceleration are used (the user may define different travel speeds at the beginning and end of a movement interval, but the acceleration is assumed constant).

The first physics relationship used is that the total time is equal to the total distance divided by the average velocity. The second physics relationship used is that the distance traveled is equal to the initial distance plus the initial velocity multiplied by the time plus one half the acceleration multiplied by the time squared. Once the total time is calculated for the interval. it must be rounded off to a discreet number of time steps. After dividing the total time by the time step interval, the number of steps are rounded up (if the remainder is greater than or equal to 0.5 ) or rounded down (if the remainder is less than 0.5 ).

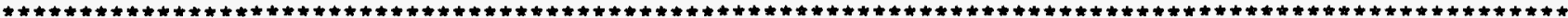
ave vel = (initial_vel + final_vel) / 2

delta_vel a final_vel - initial_vel

time Eotal. = total s / ave vel

number_steps $=$ time_total 7 time_step

If number_steps - Int (number_steps) $>0.5$ Then number_steps = Int (number_steps) + 1 Else number_steps = Int (number_steps)

accel = delta_vel / time_total

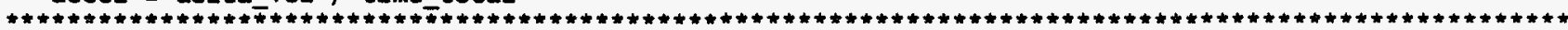

Use a loop to insert one row at a time and fill in data for each time step in the interval.

As each row is inserted, the rest of the rows are shifted down so that the variable called

"current_row" refers to the inserted (empty) row, and "current row + In $^{\text {n }}$ is always the final row of

the interval. The total number of rows to be inserted during the loop execution equals one less

than the number of steps, because the last step is the final row of the interval.

The new sx' coordinate for the inserted row is called s new and is calculated from the physics equation with time equal to the number of time steps that have occured. The sy' coordinate is constant and is equal to the same value used in the previous row. The angle alpha needs to be calculated for movements under a cable.

For $k=1$ To number steps - 1 step 1

s_new $=s$ initial + initial_vel $* k *$ time_step + accel $/ 2 *\left(k \cdot\right.$ time_step) $\wedge^{2}$

Range (CelIs (current_row, 1): Cells (current_row, 36)).Activate

Selection. Insert shift: $=x$ IDown

Cells (current row, 3 ) $=3$ new

Cells (current row, 4 ) $=$ Cells (current row $-1,4$ )

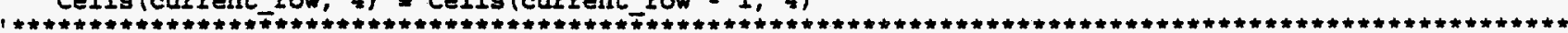

Determine if the movement is going forward under a cable by looking for the designator called

"Farthest point fwd under cable". If so, do the following. If not, then skip the next steps by going to the step in the subroutine called "not_fwd_under cable".

If the movement is under a cable, then the values for alpha need to be calculated. Because the current row was inserted as an empty row, the cable data must be read from "current row +1 "

(the final row of the interval). The variables $a, b$, and $c$ are used in the quadratic formula to solve for the cosine of the angle alpha. The cosine of alpha is then written into column 19, and the angle alpha (in radians) is evaluated in column 20. The values for the "keep out diameter" around the cable, the sx' and sy' coordinates to the center of the cable, the distance "delta_s" under the cable and the angle alpha (converted to degrees) are then filled in for the row.

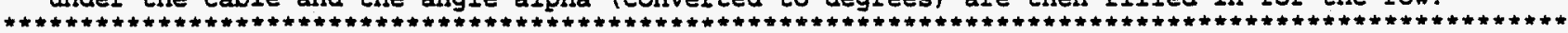

If Cells (current_row $+1,1$ ) « "Farthest point fwd under cable" Then GoTo not_fwd_under_cable delta_s a Abs (s_ñew - s_initial)

syb $=$ Cells (current_row, 4 )

dia $=$ Celis (current_row $+1,7$ ) 


\section{Module2}

sxcb $\approx$ Cells (current_row $+1,8$ )

sycb = Cells (current_row $+1,9$ )

$\mathrm{H}=\mathrm{Abs}($ sycb - syb)

$a$ (delta $s-\left(\right.$ dia + beam) $/$ 2)^ $2+\mathrm{H}^{\wedge}$

$b=2 *($ día + beam) $/ 2 *$ (delta $s-(d i a+$ beam) / 2)

$c=((d i a+b e a m) / 2) \wedge 2-H \wedge \overline{2}$

cos alpha $=(-b+\operatorname{sqr}(b-2-4 * a \cdot c)) /(2 * a)$

CelIs (current_row, 19) = cos_alpha

Cells (current_row, 20) $="=A \operatorname{COS}(\mathrm{RC}[-1]) "$

Cells (current row, 7) $=$ dia

Cells (current row, 8) = sxcb

Cells (current_row, 9) = sycb

Cells (current_row, 10) = delta $s$

Cells (current_row, 11) = CellsTcurrent_row, 20) $180 / 3.14159265358979$

not_fwd_under_cabie:

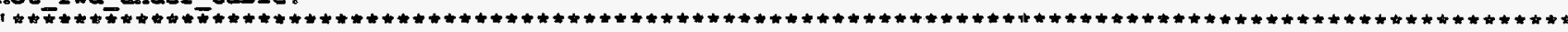

Determine if the movement is going backward under a cable by looking for the designator called "Farthest point bkwd under cable". If so, do the following. If not, then skip the next steps by going to the step in the subroutine called "not_bkwd_under_cable" .

If the movement is under a cable, then the values for alphā need to be calculated. Because the current row was inserted as an empty row, the cable data must be read from "current row + " (the final row of the interval). The variables $a_{b} b$, and $c$ are used in the quadratic formula to solve for the cosine of the angie alpha. The cosine of alpha is then written into column 19 , and the angle alpha (in radians) is evaluated in column 20. The values for the "keep out diameter" around the cable, the sx' and sy' coordinates to the center of the cable, the distance "delta_s" under the cable and the angle alpha (converted to degrees) are then filled in for the row.

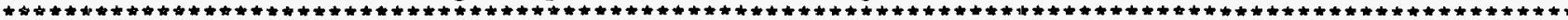

If Cells (current_row - k, 1) « "Farthest point bkwd under cable" Then GoTo not_bkwd_under_cable

delta_s a Abs (s_ñew - s_final)

syb $=$ - Cells (current_row, 4 )

dia = Cells (current row $+1,7$ )

sxcb $=$ Cells (current_row $+1,8$ )

sycb = Cells (current_row $+1,9$ )

H a Abs (sycb - syb)

$a=$ (delta $s$ - (dia + beam) $/ 2$ 2) $2+\mathrm{H} \wedge$

b = $2 *$ (día + beam) /2: (delta $s-(d i a+b e a m) / 2$ )

$c=($ (dia + beam) $/ 2) \wedge 2-H \wedge \overline{2}$

$\cos$ alpha $=(-b+\operatorname{Sqr}(b \wedge 2-4 \cdot a \cdot c)) /(2 * a)$

CelIs (current_row, 19) = cos_alpha

Cells (current_Low, 20) $=$ "=ACOS(RC $[-1]$ )

Cells (current row, 7) = dia

Cells (current_row, 8) = sxcb

Cells (current_row, 9 ) = sycb

Cells (current row, 10) = deltas

Cells (current row, 11) $=$ Cells (current row, 20) $180 / 3.14159265358979$

not_bkwd_under_cable:

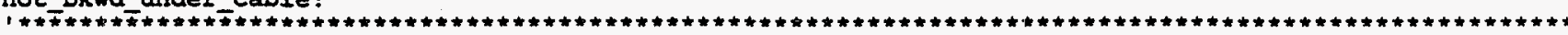

1. Increment the row number and run through the loop again

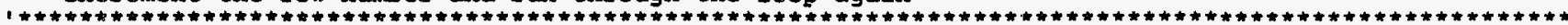
current_row $=$ current_row +1 Next $k$

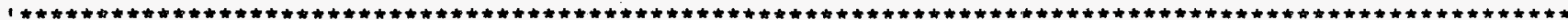
Increment the row number and return to the main subroutine called "Move"

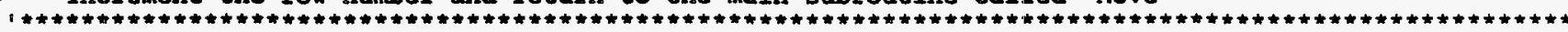
current row $=$ current row +1

End Sub

\section{Sub Move_delta_y()}

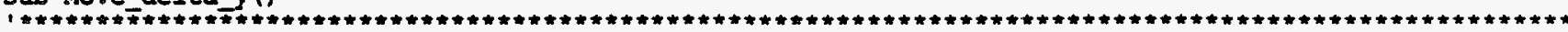
Activate Sheet 3

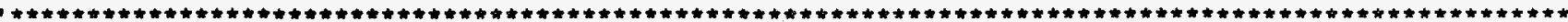
Worksheets ("Sheet3"). Activate

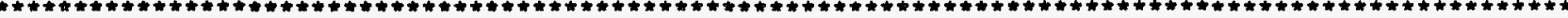
Read in the values for the initial sy coordinate value and the final sy' coordinate value in the interval. Calculate the total distance to move. Compare the values for the initial and final sy' coordinates to determine if the velocity is in the positive or negative directions. Then set the initial and final velocities equal to either plus or minus the travel speed (column 6 ) values.

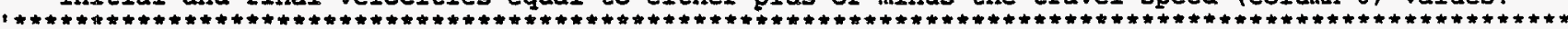
s_initial $=\operatorname{Cells}$ (current row $=1,4$ )

$s_{\text {Einal }}=$ Cells (current row, 4)

tōtal $s=s$ final - s iñitial

If $s$ final $>s$ initiaI Then initial_vel = Cells (current_row - 1, 6) Else

initial vel $=-1$ * Cells (current row $-1,6$ ) 6)

If s_final > s_initial Then final_vel = Cells(current_row, 6) Else final_vel = - 1 * Cells(current_row

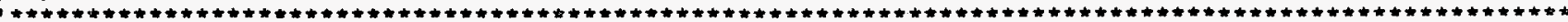

Determine if this is the special case for a guick movement around the cable with the shutter
} 
Module2

turned off by checking for the labels designating the farthest points forward or backward under the cable have been reached. If so, the velocity of movement is not governed by the values entered for the travel speed (column 6). In these cases, the travel speed value is used to determine the final velocity of the previous movement. Both the initial and final velocities while moving with the shutter off are set to the value calculated by dividing the distance to travel during this move by the value of the "Time to back under cable" (column 12).

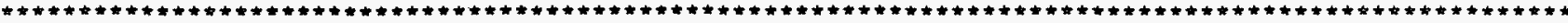
If Cells(current row, 1) = "Farthest point bkwd under cable" Then initial_vel = (s_final - s_initial) / Cells (current_row, 12)

If Cells (current_row, 1) = "Farthest point bkwd under cable" Then final_vel = initial_vel If Cells (current_row - 1, 1) = "Farthest point fwd under cable" Then initial_vel = (s_final - s_initial) / Cells (current_row - 1, 12)

If Cells (current_row $-1,1)=$ "Farthest point fwd under cable" Then final_vel = initial_vel

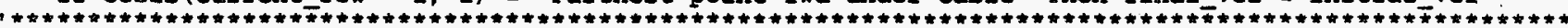
Because the velocity can change over an interval, the equations from physics for a body moving under constant acceleration are used (the user may define different travel speeds at the beginning and end of a movement interval, but the acceleration is assumed constant).

The first physics relationship used is that the total time is equal to the total distance divided by the average velocity. The second physics relationship used is that the distance traveled is equal to the initial distance plus the initial velocity multiplied by the time plus one half the acceleration multiplied by the time squared.

once the total time is calculated for the interval. It must be rounded off to a discreet number of time steps. After dividing the total time by the time step interval, the number of steps are rounded up (if the remainder is greater than or equal to 0.5 ) or rounded down (if the remainder is less than 0.5 ).

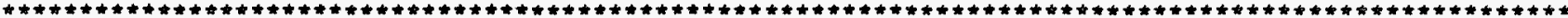

ave vel $\Rightarrow$ (initial_vel + final_vel) / 2

delta_vel = final vel - initial_vel

time Eotal a totaI s / ave vel

numbër_steps = timé_total 7 time_step

If number_steps - Int (number_stepss) $>0.5$ Then number_steps = Int (number_steps) + 1 accel $=$ delta_vel / time_totäl

Use a loop to insert one row at a time and fill in data for each time step in the interval.
As each row is inserted, the rest of the rows are shifted down so that the variable called

"current row" refers to the inserted (empty) row, and "current row + I" is always the final row of the interval. The total number of rows to be inserted during the loop execution equals one less than the number of steps, because the last step is the final row of the interval.

The new sy' coordinate for the inserted row is called s new and is calculated from the physics equation with time equal to the number of time steps that have occured. The sx' coordinate is constant and is equal to the same value used in the previous row. The angle alpha needs to be calculated for movements under a cable.

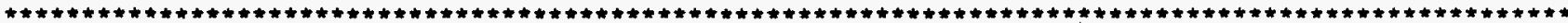
For $k=1$ To number_steps - 1 Step 1

s_new $=s$ initial + initial_vel $* k \cdot$ time_step + accel $/ 2 \cdot(k *$ time_step) 2

Ränge (Celis (current row, 1), Cells (current_row, 36)). Activate

Selection. Insert Shift: =xlDown

Cells (current_row, 4 ) $=9$ new

Cells (current_row, 3) $=$ Célls (current_row - 1, 3)

Determine if the movement is going forward under a cable by looking for the designator called "Farthest point fwd under cable". If so, do the following. If not, then skip the next steps by going to the step in the subroutine called "not fwd under cable"

If the movement is under a cable, then the values för alpha need to be calculated. Because the current row was inserted as an empty row, the cable data must be read from "current row + 1 " (the final row of the interval). The variables $a, b$, and $c$ are used in the quadratic formula to solve for the cosine of the angle alpha. The cosine of alpha is then written into column 19. and the angle alpha (in radians) is evaluated in column 20. The values for the "keep out diameter" around the cable, the sx' and sy' coordinates to the center of the cable, the distance "delta_s" under the cable and the angle alpha (converted to degrees) are then filled in for the row.

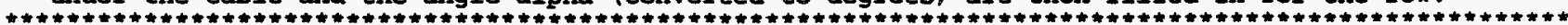
If Cells(current_row $+1,1) \longleftrightarrow$ "Parthest point fwd under cable" Then GoTo not_fwd_under_cable delta_s = Abs (s_ñew - s_initial)

sxb $=$ Cells (curzent row. 3 )

dia $=$ Cells (current_row $+1,7$ )

sxcb $=$ Cells (current_row $+1,8$ )

sycb = Cells (current row $+1,9$ )

$H=A b s(s \times c b-s x b)$

$a=($ deltas $-($ dia + beam $) / 2) \wedge 2+H^{\wedge} 2$

$b=2 \cdot(d \bar{i} a+b e a m) / 2 *$ (delta_s $-(d i a+b e a m) / 2$ )

$c=((d i a+b e a m) / 2) \wedge 2-H \wedge \overline{2}$

cos alpha $=(-b+\operatorname{sgr}(b-2-4 * a * c)) /(2 * a)$

Celis (current_row, 19) = cos_alpha

Cells (current-row, 20) $="=A \operatorname{COS}(R C[-1]) "$

Cells (current row, 7) = dia

Cells (current_row, 8) = sxcb

Cells (current_fow, 9) = sycb

Cells (current_row, 10) = delta_s 
Cells (current_row, 11) $=$ Cells (current_row, 20) * $180 / 3.14159265358979$

not_fwd under_cabIe:

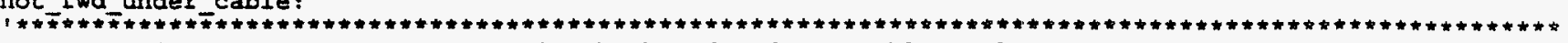

Determine if the movement is going backward under a cable by looking for the designator called

"Farthest point bkwd under cable". If so, do the following. If not, then skip the next steps by going to the step in the subroutine called "not bkwd under cable".

If the movement is under a cable, then the valuess fo $\vec{x}$ alphā need to be calculated. Because the current row was inserted as an empty row, the cable data must be read from "current row + 1 " (the final row of the interval). The variables $a, b$, and $c$ are used in the quadratic formula to solve for the cosine of the angle alpha. The cosine of alpha is then written into column 19, and the angle alpha (in radians) is evaluated in column 20 . The values for the "keep out diameter" around the cable, the sx' and sy' coordinates to the center of the cable, the distance "delta_s" under the cable and the angle alpha (converted to degrees) are then filled in for the row.

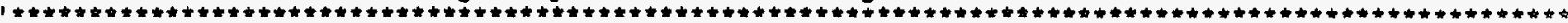
If Cells(current_row - $k$, I) 〈 "Farthest point bkwd under cable" Then GoTo not_bkwd_under_cable delta_s = Abs (s_new - s_final)

$\mathbf{s x b}=-$ Cells (current row, 3 )

dia $=$ Cells (current row $+1,7$ )

$s \times c b=$ Cells (current_row $+i, 8$ )

sycb = Cells (current row $+1,9$ )

$H=A b s($ sxcb $-\mathbf{s x b})$

$a=($ delta $s-($ dia + beam $) / 2$ 2) $2+H \wedge 2$

$\mathrm{b}=2 *(\mathrm{~d} \overline{\mathrm{i}} \mathbf{a}+\mathrm{beam}) / 2 *(\mathrm{delta} s-(\mathrm{dia}+$ beam) $/ 2$ )

$c=(($ dia + beam $) / 2) \wedge 2-\mathrm{H}^{\wedge} \overline{2}$

cosalpha $=(-b+5 q r(b \wedge 2-4 \cdot a * c)) /(2 \bullet a)$

CelIs (current row, 19) = cos alpha

Celis (current row, 20) $="=A \operatorname{COS}(R C[-1]) "$

Cells (current_row, 7) = dia

Celis (current_row, 8) = sxcb

Celis (current row, 9) $=$ sycb

Cells (current row, 10) = delta_s

Cells (current_row, 11) = CellsTcurrent_row, 20) $\bullet 180 / 3.14159265358979$

not bkwd under cable:

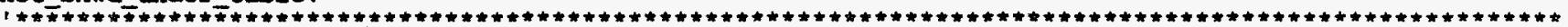
Increment the row number and run through the loop again

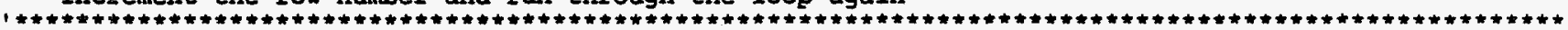
current_row $=$ current_row +1 Next $k$

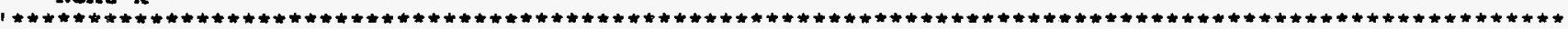

Increment the row number and return to the main subroutine called "Move"

‘ End Sub current_row $=$ current_fow +1

Sub Move_corner ()

i

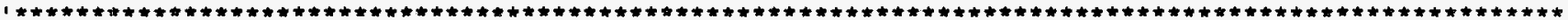
Worksheets ("Sheet 3 ") . Activate

Read in the values for the initial sx' and sy' coordinates and the final sx' and sy' coordinates in the interval. The $x$ digtance and $y$ distance to the center of the radiused corners are half the length minus the radius and half the width minus the radius respectively. The sx' and sy' coordinates to the center of the radiused corners are calculated and labeled "xcb" and "ycb" and are either positive or negative depending on the initial sx' and sy' coordinates.

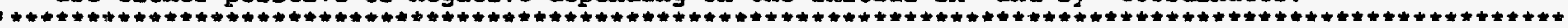
sxb initial $=$ Cells (current row $-1,3$ )

syb_initial = Cells (current_row - 1, 4)

sxb-final Cells (current row, 3)

syb final $=$ Cells (current row 4 )

If $\overline{s x b}$ initial $>0$ Then $x \overline{c b}=$ lngth $/ 2$ - radius Else $x c b=-$ (lngth $/ 2$ - radius) If $\mathrm{syb}$-initial $>0$ Then $\mathrm{ycb}=$ wdth $/ 2$ - radius Else ycb $=-$ (wdth $/ 2$ - radius)

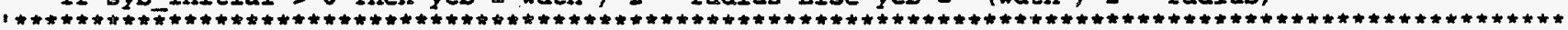
In the corners, the $\mathbf{s x}^{\prime}$ and $\mathbf{s y}^{\prime}$ coordinates are related by the angle though the corner called "theta". The value for "theta inital" and "theta_final" are calculated (using absolute values), and are always either 0 or pi $\overline{2}$ radians (if "theta $\overline{a n i t a l " ~}=0$ then "theta_final" $=\mathrm{pi} / 2 \mathrm{radians}$ and vice versa). The arc tangent method was used to calculate the theta values because this version of Visual Basic doesn't support arc cosines or arc sines. Because of this, a small number was added to the denominator to prevent division by zero. The "total theta" value (difference between the initial and final theta values) will always be either plus or minus pi/2 and the angular velocities "theta dot inital" and "theta dot final" will have the same positive or negative sign as the value for "total Eheta". The initial and final velocities are still based on the user input value for travel speed (column 6), but these travel speed values are used to determine the corresponding angular velocities "theta dot" based on the relationship that the tangental velocity (travel speed) is equal to the angular velocity multiplied by the radius. Using this relationship. 
the initial and final angular velocities "theta_dot_initial" and "theta_dot_final" are calculated as either plus or minus the travel speed divided by the radius.

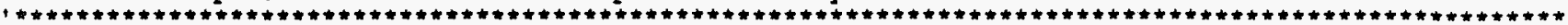

theta_initial $=$ Atn (Abs (syb_initial - ycb) / (Abs (sxb_initial - xcb) +0.0000000000001))

theta_final $=$ Atn (Abs (syb_final - ycb) /(Abs (sxb_final - xcb) +0.00000000000001))

total theta = theta final- theta initial

If total_theta $>0$ Then theta_dot_initial $=$ Cells(current_row - 1,6) / radius Else -

theta_dot_initial $=-1 \cdot$ Cells (current_row $-1,6$ ) $/$ radiūs

If total_theta $>0$ Then theta_dot_final $=$ Cells (current_row, 6) / radius Else theta_dot_final $=-1$ Cells (Eurrent_row, 6) $/$ radius

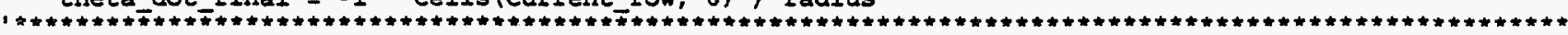
Because the velocity can change over an interval, the equations from physics for a body moving under constant accelexation due to a constant angular acceleration are used (the user may define different travel speeds at the beginning and end of a movement interval, but the acceleration is assumed constant). The first physics relationship used is that the total time is equal to the total theta distance "total_theta" divided by the average angular velocity "ave theta_dot". The second physics relationship used is that the angle traveled is equal to the initial angle plus the initial angular velocity multiplied by the time plus one half the angular acceleration multiplied by the time squared.

Once the total time is calculated for the interval, It must be rounded off to a discreet number of time steps. After dividing the total time by the time step interval, the number of steps are rounded up (if the remainder is greater than or equal to 0.5 ) or rounded down (if the remainder is less than 0.5 ).

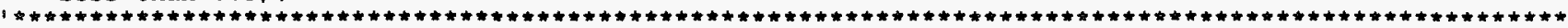
ave theta dot $=$ (theta dot initial + theta dot final) $/ 2$

delta theta dot $=$ theta dot final - theta dot initial

time_total = total_thetā/,ave_theta_dot

number_steps = time total $/$ time_step

If number steps - Int (number steps) $>0.5$ Then number steps = Int (number steps) + 1 Else number_steps = Int (number_stêps)

theta_double dot $=$ delta theta dot $/$ time total

1

Use a loop to insert one row at a time and fill in data for each time step in the interval.

As each row is inserted, the rest of the rows are shifted down so that the variable called

"current row" refers to the inserted (empty) row, and "current row + In is always the final row of

the interval. The total number of rows to be inserted during the loop execution equals one less

than the number of steps, because the last step is the final row of the interval.

The new theta value used to calculate the sx' and sy' coordinates for the inserted row is called

"theta new" and is calculated from the physics equation for theta with time equal to the number of

time sEeps that have occured. Once the new value for theta is calculated, the new sx' and $s y^{\prime}$

coordinates for the inserted row (called "sxb new" and "syb new" respectively) are calculated

using the coordinates of the radius center, the radius, and the angle theta. The value called

"sign $x$ " or "sign_y" are used to correct the equation for use on all cormers of the part.

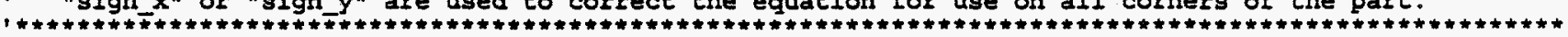

For $k=1$ To number steps - 1 step 1

theta_new $=$ theta_iñitial + theta_dot_initial $\bullet k$ * time_step +

theta_double_dot $72 \cdot\left(k \cdot t i m e \_\bar{s} t e p\right) \wedge 2$

If $x c b>0$ Then sign $x=1$ Else sign $x=-1$

If $y \mathrm{cb}>0$ Then sign_y $=1$ Else sign_y $=-1$

sxb_new $=\mathrm{xcb}+\operatorname{sign} \mathrm{x}$ - radius $* \cos _{\text {(theta_new) }}$

syb-new $=y c b+\operatorname{sign} y$ - radius $\sin ($ theta new)

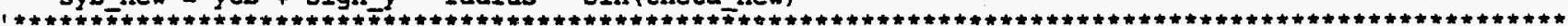

Insert a row and fill in the calculated values for the sx' and sy' coordinates

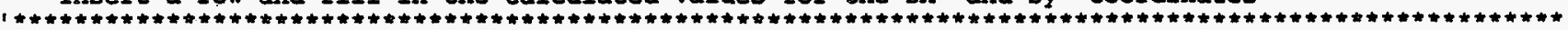

Range (Cells (current_row, 1), Cells (current_row, 36)) .Activate

Selection. Insert Shift: $=x$ lDown

Cells (current row, 3 ) $=$ sxb new

Cells (current row, 4) = syb new

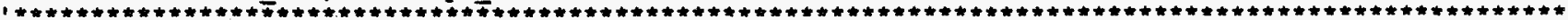

Increment the row number and run through the loop again

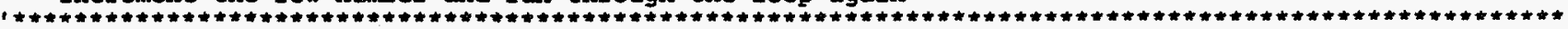
current row $=$ current row +1

Next $k$

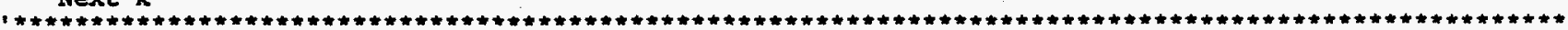

Increment the row number and retum to the main subroutine called "Move"

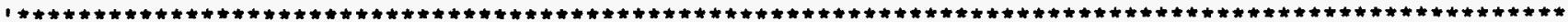
End Sub

current_row $=$ current_row +1

Sub fillin_beta()

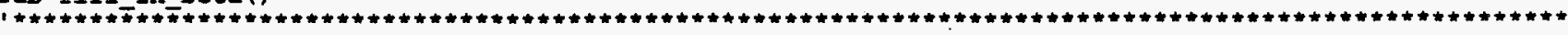

Activate Sheet 2

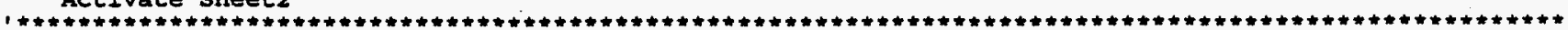


Worksheets ("Sheet2") .Activate

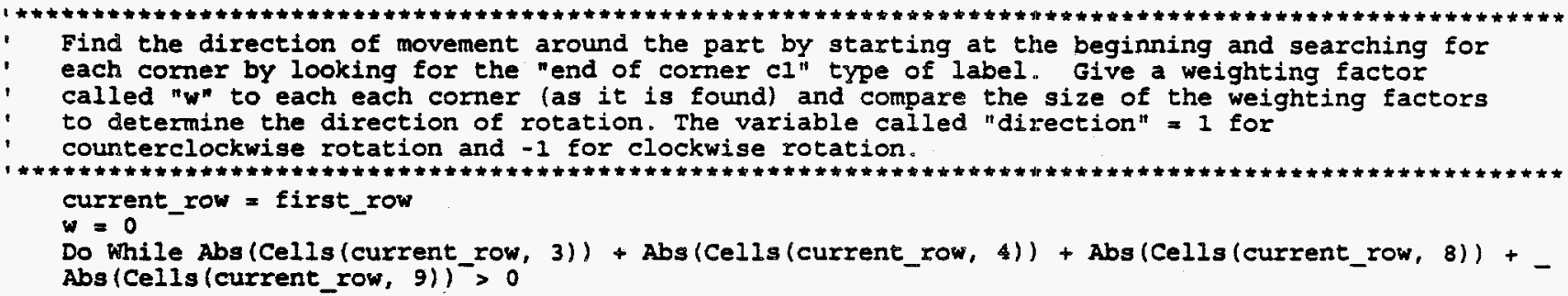
If Cells (current_row, 1) = "end of corner c1" or Cells (current_row, 1) = "end of corner c2" or Cells(current_row, 1 ) $=$ "end of corner c3" Or Cells(current_row, 1 ) = "end of cormer c4" Then $w=w+1$

If Cells (current_row, 1) $=$ "end of corner c1" Then $c 1=w$ If Celis (current_row, 1 ) $=$ "end of corner $c 2$ " Then $c 2=w$ current row $=$ current row +1

Loop

If $c 1>c 2$ Then direction $=1$

If $c 1<C 2$ And $c 1=1$ And $c 2=4$ Then direction $=1$

If $c 1>c 2$ And $c 2=1$ And $c 1=4$ Then direction $=-1$

If direction $<1$ Then direction $=-1$

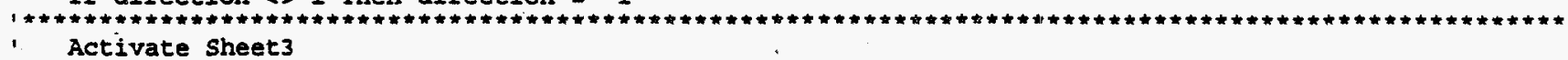

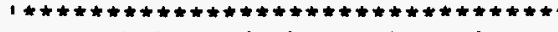

Worksheets ("Sheet 3 "). Activate

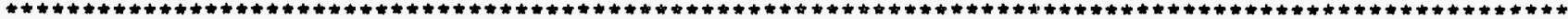
Start at the second row and run the major loop used to fill in the beta values until the end of the data is found. Reset the variable "interval" to zero each time through the loop

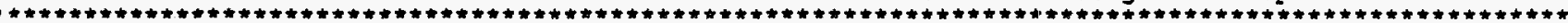
current_row $=$ first_row +1

Do While Abs (Cells (current_row, 3)) + Abs (Cells (current_row, 4)) >0 interval $=0$

ił Look for a cell with out a value for beta. When such an empty cell is found, increment the variable called "interval" and then check for a beta value in the next row. Do this until a beta value is found (the number of empty beta cells is the final value of "interval").

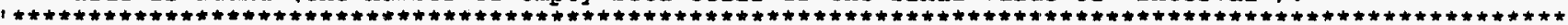
Do While IsEmpty (Cells (current_row, 5)) = True

interval = interval + 1

current_row $=$ current_row +1 Loop

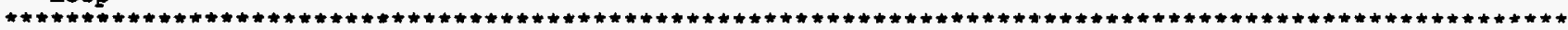
Name the row just before the empty interval "starting_row" and the curxent row (just after the empty intervall as "ending_row".

Calculate the incremental step for beta "delta_beta" as the change in beta values over the interval divided by the size of the interval (number of empty rows plus one). Make the current row the first row in the empty beta interval.

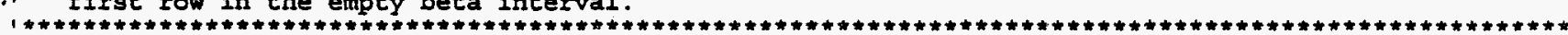
starting row $=$ current row - interval - 1

ending rō $=$ current rō

delta_beta $=($ Cells (ending_row, 5) - Cells (starting_row, 5)) $/$ (interval + 1) current_zow $=$ starting_row +1

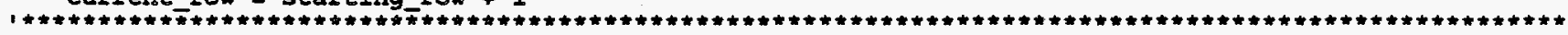
Run a loop to fill in the beta values incrementing each beta by adding the "delta beta" value

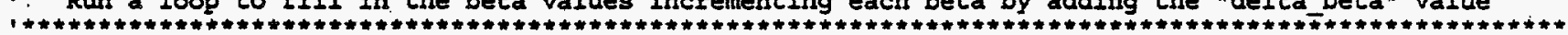
For $k=1$ To interval step 1

Cells (current row, 5 ) = Cells (starting row, 5 ) $+k$ * delta beta

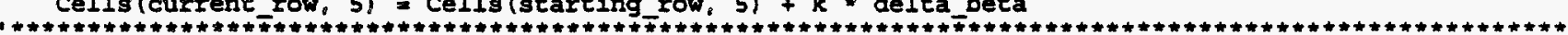
Check whether the interval includes forward movement under a cable. If so, set the variable called "fwd" to be true. If "fwd" is true, then determine over which edge of the part, the movement under the cable occurs.

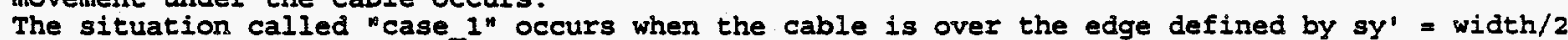
(verified by a positive sy ${ }^{\top}$ value and the absolute value of the sx' value less than the length/2). The situation called "case 2 " occurs when the cable is over the edge defined by $3 x^{\prime}=$ length/2 (verified by a positive $s x^{\top}$ value and the absolute value of the sy' value less than the width/2). The situation called "case 3 " occurs when the cable is over the edge defined by sy' $x-$ width/2 (verified by a negative sy value and the absolute value of the sx' value less than the length/2). The situation called "case 4 " occurs when the cable is over the edge defined by $\mathbf{s x}$ ' $=-1$ ength/2 (verified by a negative sx' value and the absolute value of the sy' value less than the width/2). For each case, the direction of rotation of the part determines the value of beta which will violate the "keep out dia" of the cable. This value is called "beta max". If the beta value previousiy calculated violates the "beta_max" restriction then the betta value will be changed to the "beta max" value. 
Module2

If Cells (ending_row, 1 ) = "Farthest point fwd under cable" Then fwd = True Else fwd = False

If fwd = True And Cells (current_row, 4) >0 And Abs (Cells (current_row, 3 )) < lngth / 2 Then case_1 $1=$ True Blse case_1 $1=$ False

If case_1 = True Then beta_max $=0$ - direction * Cells (current_row, 11)

If case_I $_{1}=$ True And direction $=1$ And beta_max $<$ Cells (current_row, 5) Then Cells (current_row, 5) = beta_max

If case_1 = True And direction $=-1$ And beta_max > Cells (current_row, 5) Then Cells (current_row, 5) = beta_max

If fwd = True And Cells (current_row, 3) >0 And Abs (Cells (current_row, 4)) < wdth $/ 2$ Then case_2 = True Else case_2 = False

If case_2 = True Then beta_max $=90$ - direction Cells (current_row, 11)

If case_2 $=$ True And direction $=1$ And beta_max < Cells (current_row, 5) Then Cells (cürrent_row, 5) abeta_max

If case_2 = True And direction = -1 And beta_max > Cells (current_fow, 5) Then Cells (cürrent_row, 5) = beta_max

If fwd $=$ True And Cells (current_row, 4) < 0 And Abs(Cells (current_row, 3)) < lngth $/ 2$ Then case_3 = True Else case_ 3 = False

If case_3 $=$ True Then beta_max $=180$ - direction * Cells (current_row, 11)

If case 3 = True And direction $=1$ And beta_max < Cells (current_row, 5) Then -

Cells (current_row, 5) = beta max

If case_3 = True And direction $=-1$ And beta_max > Cells (current_row, 5) Then -

Cells (current_row, 5 ) = beta_max

If fwd = True And Cells (current_row, 3) < 0 And Abs (Cells (current_row, 4)) < wdth $/ 2$ Then case_4 = True Else case_4 = False

If case 4 = True Then beta $\max =270$ - direction - Cells (current row, 11)

If case_4 = True And direction = 1 And beta_max < Cells (current_row, 5) Then -

Cells (cürrent_zow, 5) = beta_max

If case_4 = True And direction $=-1$ And beta_max $>$ Cells (current_row, 5) Then

Cells (current_row, 5) = beta_max

Check whether the interval includes backward movement under a cable. If so, set the variable called "bkwd" to be true. If "bkwd" is true, then determine over which edge of the part, the movement under the cable occurs.

The situation called "case I" occurs when the cable is over the edge defined by sy' $=$ width $/ 2$

(verified by a positive sy' value and the absolute value of the sx' value less than the length/2). The situation called "case 2 " occurs when the cable is over the edge defined by $9 x$ ' $=1$ ength/2 (verified by a positive $3 x^{\top}$ value and the absolute value of the sy' value less than the width/2). The situation called "case_3" occurs when the cable is over the edge defined by sy' =-width/2 (verified by a negative sy value and the absolute value of the sx' value less than the length/2). The situation called "case 4 " occurs when the cable is over the edge defined by $s x^{\prime}=-1$ ength/2 (verified by a negative sx' value and the absolute value of the sy' value less than the width/2). For each case, the direction of rotation of the part determines the value of beta which will violate the "keep out dia" of the cable. This value is called "beta max". If the beta value previously calculated violates the "beta max" restriction then the beta value will be changed to the "beta max" value.

If Cells(starting row, I) " "Farthest point bkwd under cablen Then bkwd = True Else bkwd False

If bkwd = True And Cells (current_row, 4) >0 And Abs (Cells (current_row, 3)) < Ingth / 2 Then case_ 1 = True Blse case_I = Falsē

If case_1 $1=$ True Then beta $\max =0$ + direction - Cells (current_row, 11) If case-1 1 True And direction = 1 And beta_max > Cells (current_row, 5) Then Cells (current_row, 5) = beta_max

If case_1 = True And direction = -I And beta_max < Cells (current_row, 5) Then Celis (currrent_row, 5) = beta_max

If bkwd = True And Cells (current_row, 3) >0 And Abs (Cells (current_row, 4)) < wdth / 2 Then case_2 = True Else case_2 = False

If case 2 = True Then beta max $=90$ + direction * Cells (current row, 11)

If case_2 $=$ True And direction $=1$ And beta_max $>$ Cells (current_row, 5) Then Cells (cūrrent_row, 5) o beta_max

If case_2 = True And direction = -1 And beta_max $<$ Cells (current_row, 5) Then Celis (current_row, 5) = beta_max 
If bkwd = Irue And Cells (current_row, 4)<0 And Abs(Cells(current_row, 3)) < Ingth $/ 2$ Then case_3 $=$ True Else case_3 3 False

If case 3 = True Then beta max $=180$ + direction - Cells (current: row, 11)

If case_- $3=$ True And direction $=1$ And beta_max $>$ Cells (current_row, 5) Then -

Cells (current_row, 5) = beta_max

If case_3 = True And direction $=-1$ And beta_max $<$ Cells (current_row, 5) Then Cells (current_row, 5) = beta_max

If bkwd = True And Cells (current_row, 3) < 0 And Abs (Cells (current_row, 4)) < wdth / 2 Then case_4 4 True Else case_ $4=$ False

If case $4=$ True Then beta $\max =270+$ direction - Cells (current row, 11)

If case_ $_{-}^{-}=$True And direction $=1$ And beta_max > Cells (current_row, 5) Then -

Cells (cürrent_row, 5 ) = beta_max

If case_ $4=$ True And direction $=-1$ And beta_max < Cells (current:_row, 5) Then Cells (cürent_row, 5) = beta max

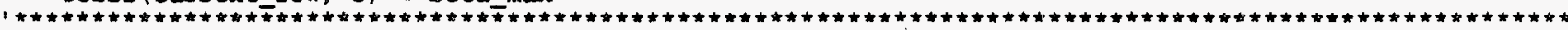
Increment the row number and run ehrough the internal loop again to fill in all beta values in the interval

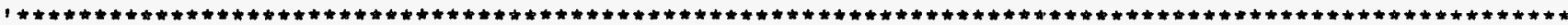
current_row $=$ current_row +1 Next $k$

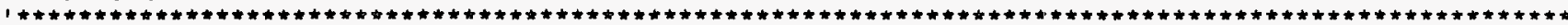
Increment the row number and run through the large loop again to find another interval without beta values

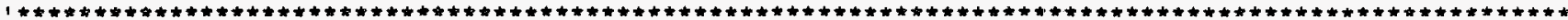
current_row $=$ current_row +1 Loop

End Sub

Sub fill in shutter()

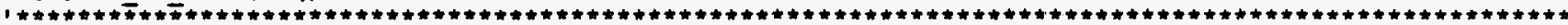
Activate Sheet 3

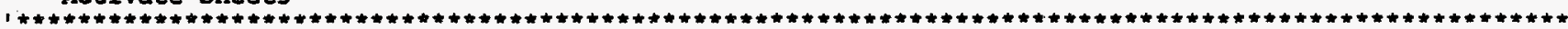
Worksheets ("Sheet 3 ") .Activate

I Start at the second row and run the major loop used to fill in the shutter values until the end of the data is found. Run the next loop when ever the shutter cell (column 13) is empty

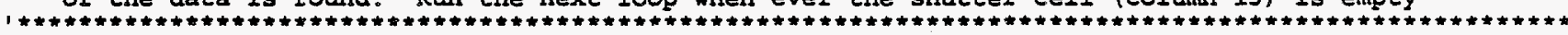
current row $=$ first row +1

Do While Abs (Celis (current row, 3)) + Abs (Cells (current row, 4)) >0

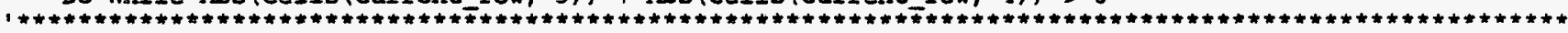
For each shutter value that is empty, fill in the shutter with the previous shutter value.

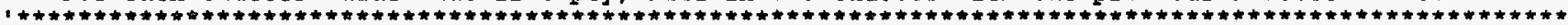
Do While IsEmpty (Cells (current_row, 13)) = True

Cells (current_row, 13) = CellsTcurrent_row - I, 13)

current row $=$ current row +1 Loop

: Increment the row number and run through the large loop again to find another interval without shutter values

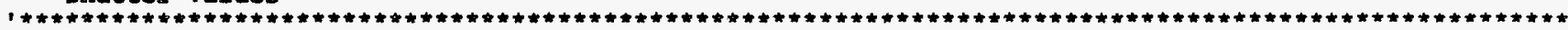
current_row $=$ current_row +1

Loop

End Sub

Cells (1, 1).Select

Sub Fill in motion data()

;

1. Activate Sheet 3

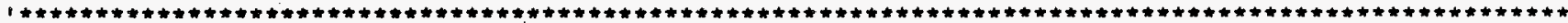
Worksheets ("Sheet 3"). Activate

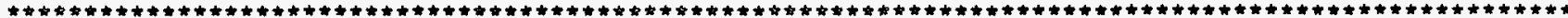
Set up area for calculating the incremental move data for driving the motion control system The first cells defined by the "first row - 1" row designation are the labels for the data columns. The next cells defined by the "first row row designation are the initial values and are set to zero in some cases (such as the Delta_x or Delta_y values). The final cells defined by the "first_row_+ 1" designation are the formulas to be used for calculating all of the subsequent values.

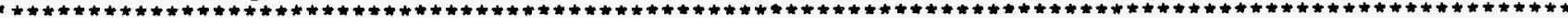

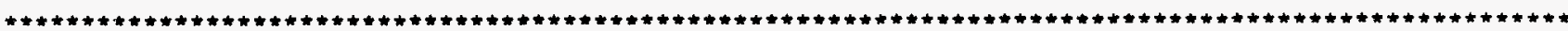
Elapsed Time (colum 21): Eirst value $=0$ subsequent values a previous value plus time step 


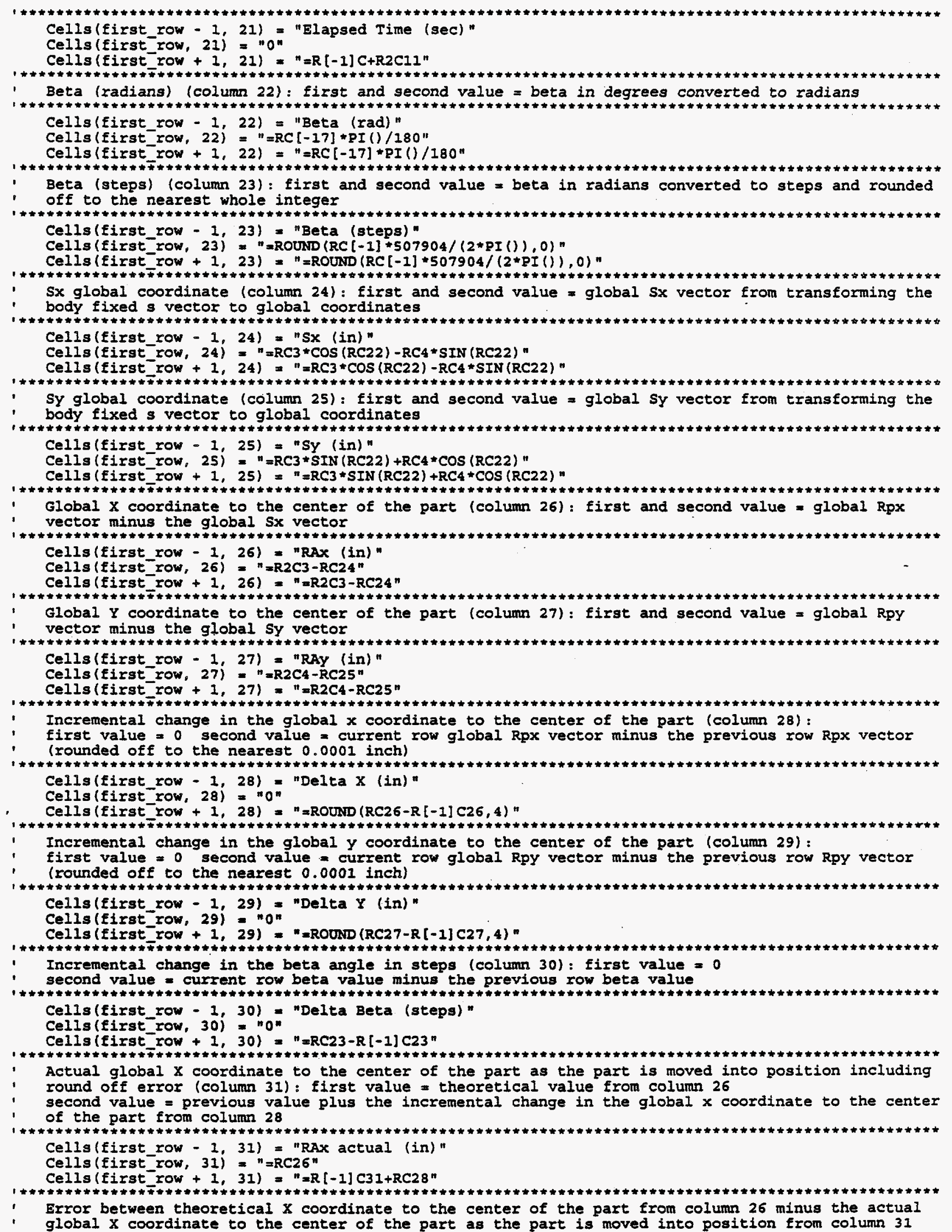


(column 32) first and second values = column $26-$ column 31

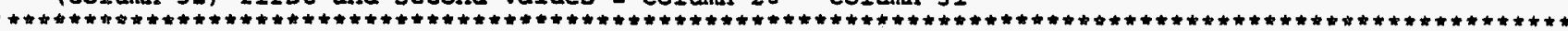
Celis (first row $-1,32$ ) $=$ "Error X (in) n

Cells (first_row, 32 ) $=$ "=RC26-RC31"

Cells (first_row $+1,32$ ) $="=$ RC26-RC31"

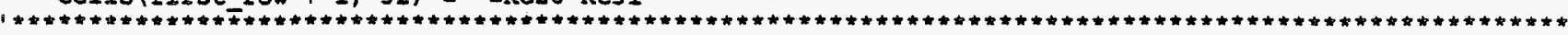

Actual global $\Psi$ coordinate to the center of the part as the part is moved into position including round off error (column 33 ): first value = theoretical value from column 27

second value $=$ previous value plus the incremental change in the global y coordinate to the center of the part from column 29

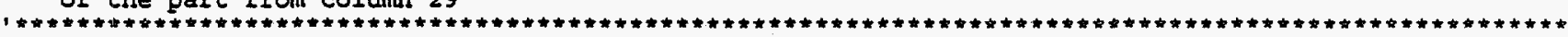
Celis (first_row $-1,33)=$ "RAy actual (in)"

Cells (first row, 33) $="=R C 27 "$

Celis (first_row $+1,33)=n=R(-1]$ C $33+R C 29 n$

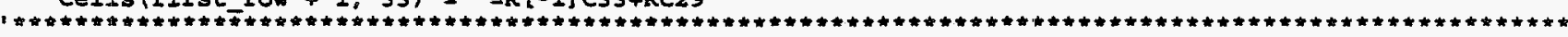
Exror between theoretical $y$ coordinate to the center of the part from column 27 minus the actual global $Y$ coordinate to the center of the part as the part is moved into position from column 33 (column 34) first and second values $=$ column $27-$ column 33

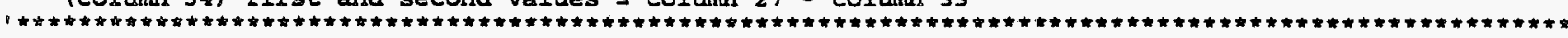
Cells (first row $-1,34$ ) = "Error $Y$ (in)"

Cells (first_row, 34) " "=RC27-RC33"

Cells (first_row $+1,34$ ) $="=\mathrm{RC27}-\mathrm{RC} 33^{n}$

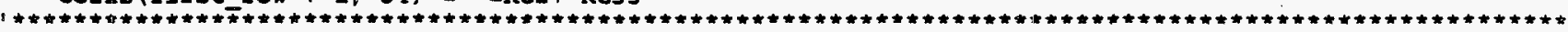

Actual beta value of the part as the part is rotated into position including round off

error (column 35) : first value = theoretical value from column 23 second value = previous value plus the incremental change in beta from column 30

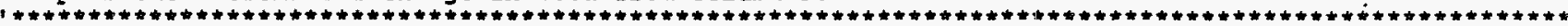
Cells (first_row $-1,35)=$ "Beta actual (steps)"

Cells (first_row, 35$)="=R C 23 "$ "

Cells (first row $+1,35)="=R[-1] C 35+R C 30 "$

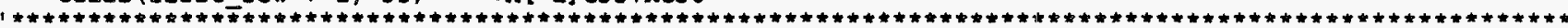
Error between theoretical beta from column 23 minus the actual beta as the part is rotated into position from column 35 (column 36) first and second values $=$ column 23 . column 35

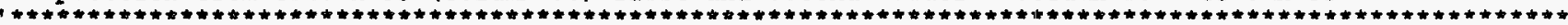
Cells (first_row - 1, 36) = "Error Beta (steps)"

Cells (first row, 36 ) $=n=R C 23-R C 35^{n}$

Cells (first row $+1,36)=n=R C 23-R C 35^{n}$

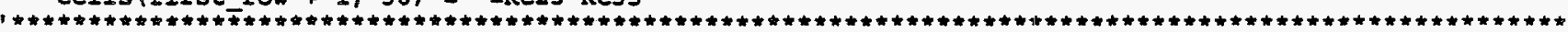
Find the end of the full data and label the last row "last_row"

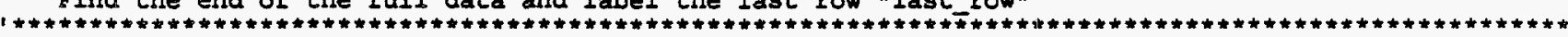
Find the end of the full data.

current_row $=$ first_row +1

Do While Abs (Cells (Current_row, 3)) + Abs (Cells (current_row, 4)) >0

current row $=$ current row $\mp 1$

last_row $=$ current_row -1 Loop

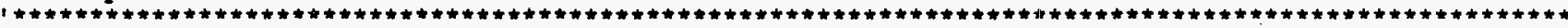
Autofill the motion control data by expanding the formulas contained in the first row plus one to a range ending with the last row. End by going to, and selecting the top corner of the sheet.

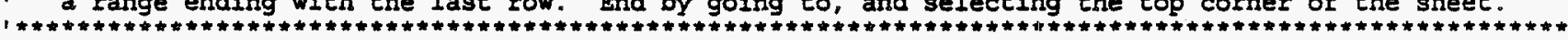
Range (Cells (first row $+1,21$ ), Cells (first row $+1,36$ )) . Select:

Selection. AutoFil] Destination:=Range (Cells (first_row $+1,21)$, Cells (last_row, 36)), Type : $=x I F i l i D e f a u l t$

End Sub

Cells (1, 1). Select

Sub Make Sheet 3 Buttong ()

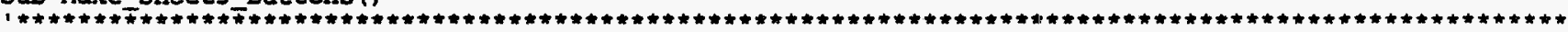
Activate Sheet 3

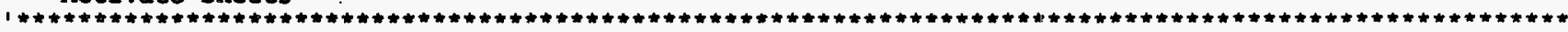
Worksheets ("Sheet3") .Activate

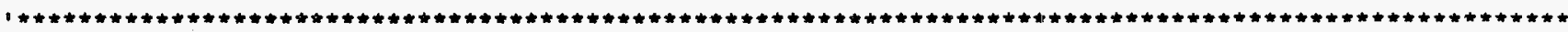
Create a button for deleting sheet three. The first commands define the button size and position, define the macro assigned to it, and the label. The rest of the commands define the way the button should look

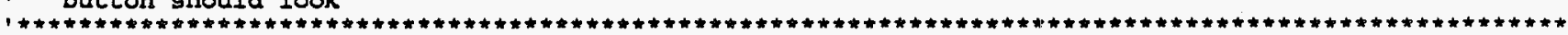

ActiveSheet. Buttons. Add (3, 10, 120,36). Select

Selection. OnAction $=$ "Delete_sheet $3 "$

Selection.Characters. Text = "Delete This Data Sheet"

With Selection. Characters (Start $:=1$, Length: $=22$ ). Font

. Name = "Times New Roman"

.Fontstyle = "Bold"

Size $=10$

.Strikethrough $=$ False

Superscript $=$ False

.Subscript $=$ False 
Module2
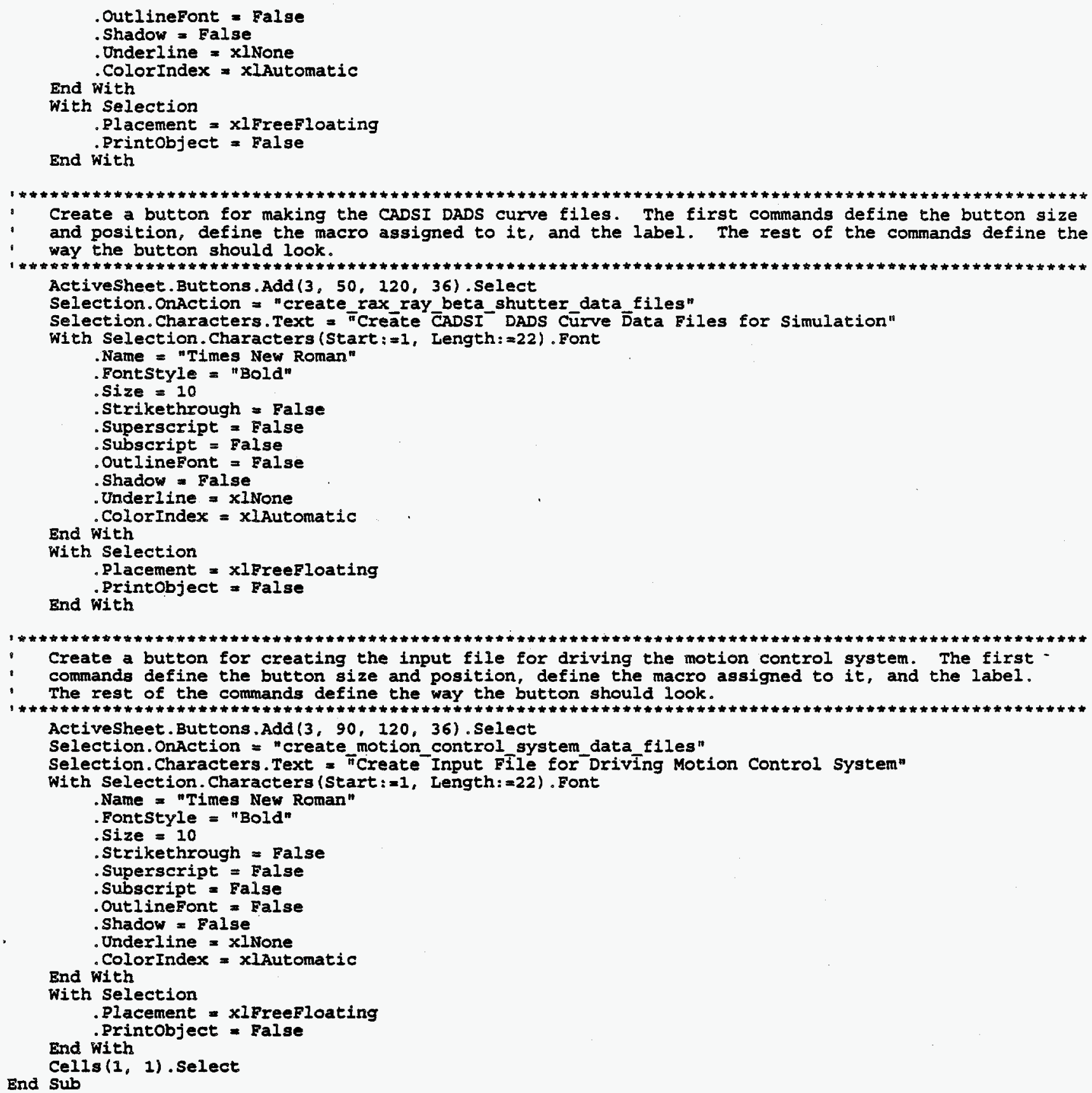
Module3

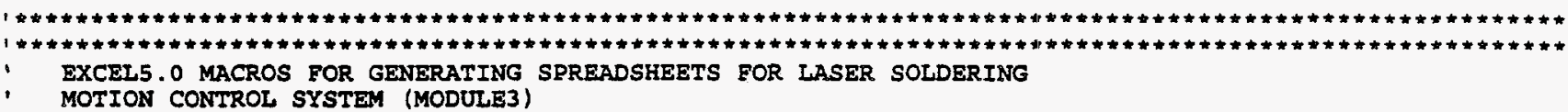

Dim sxcb As Single

Dim sycb As single

Dim time shutter off As single

Dim half beam and cable As single

Dim deltás_s_twd A $\bar{s}$ single

Dim delta_s_bkwd As single

Dim syb As Single

Dim sxb As Single

Dim cntclock As Integer

Dim direction As Integer

Sub create rax ray beta shutter data files()

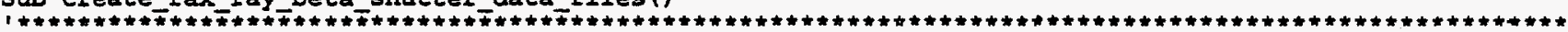

- Name a variable called "currentbook" to be the name of the current workbook so that you can return - to this workbook after activating otherg

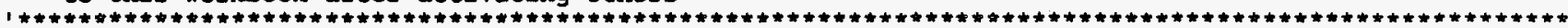
Set currentbook = Application.Activeworkbook

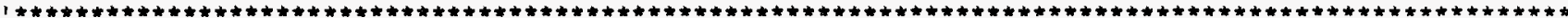
Activate the workbook called "DADS_DAT.XIS"

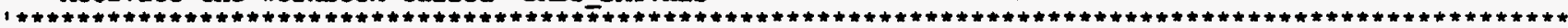
Workbooks.Open Filename: "DADS_DAT.XIS"

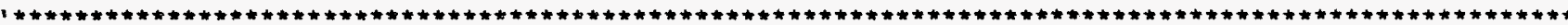

1 Delete the old data sheets from the "DADS DAT.XIS" workbook if they exist

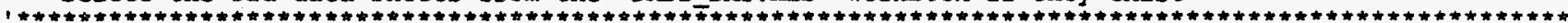
If Sheets (1). Name $=$ "rax" Then Sheets (1). Delete

If Sheets (1). Name = "ray" Then Sheets (1). Delete

If Sheets(1). Name " "beta" Then Sheets(1). Delete

If Sheets(1). Name $=$ "shutter" Then Sheets(1). Delete

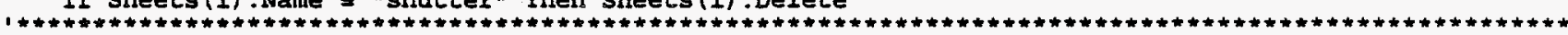

1 Create sheet "shutter"

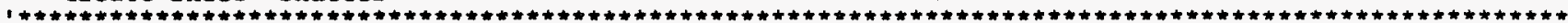
Sheets (1). Select

Sheets.Add

Sheets (1) . Name = "shutter"

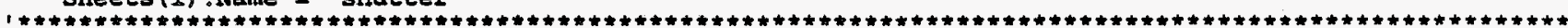
Create Sheet "beta"

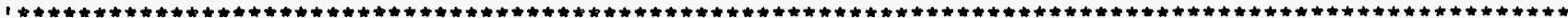
Sheets (1). Select Sheets.Add Sheets (1). Name = "beta"

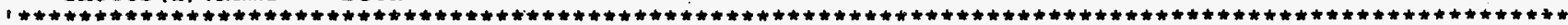
Creace sheet "ray"

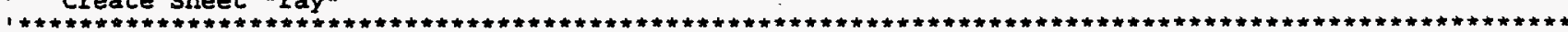
Sheets (1). Select

Sheets. Add Sheets (1). Name $=$ "ray"

1 Create Sheet "rax"

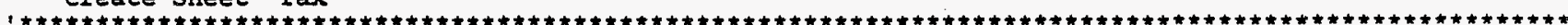
Sheets (1). Select Sheets. Add Sheets (1). Name = "rax"

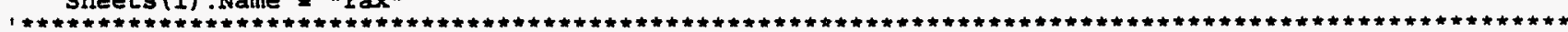

i Activate the workbook and activate Sheet3 (full data) 


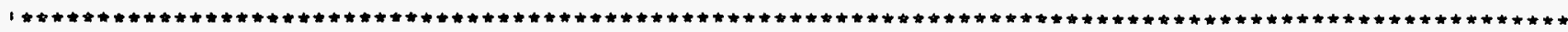

Workbooks (currentbook. Name) . Activate

Worksheets ("Sheet 3 "). Activate

1

Find end of input data

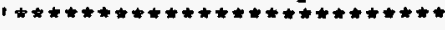

current_row $=$ first_row

Do While Abs (Cells (current_row, 3)) + Abs(Cells(current_row, 4)) >0

current_row $=$ current_row $\mp 1$

Loop

last_row = current_row

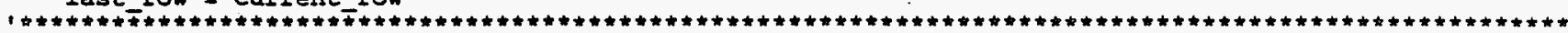
Copy elapsed time data

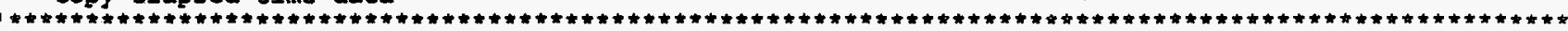
Range (Cel1s (first row, 21), Cells (last row, 21)). Select

Selection. Copy

Cells $(1,1)$. Select

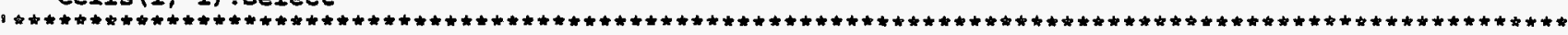
Activate the "DADS DAT.XIS" workbook and the sheets "rax" "ray" "beta", and "shutter" and paste elapsed time values.

:

Workbooks ("DADS DAT.XLS"). Activate

Worksheets ("rax"). Activate

Cells $(1,1)$. Select

Selection. PasteSpecial Paste:=xlvalues, Operation:=xlNone, SkipBlanks : =False, Transpose : =False

Worksheets ("ray"). Activate

Cells (1, 1). Select

Selection.Pastespecial Paste:=xlvalues, Operation:=xlNone SkipBlanks: =False, Transpose : =False

worksheets ("beta"). Activate

Cells (1; 1). Select

Selection.PasteSpecial Paste: =xlValues, Operation: =xlNone, SkipBlanks: =False, Transpose : =False

Worksheets ("shutter") . Activate

Cells (1, 1). Select

Selection.Pastespecial Paste:=xlValues, Operation:=xlNone, SkipBlanks:=False, Transpose: =Ealse

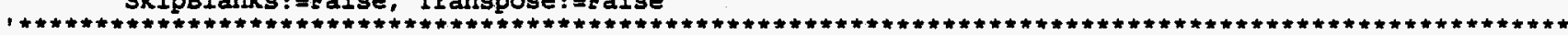

Activate the workbook and sheet 3 and copy rax data.

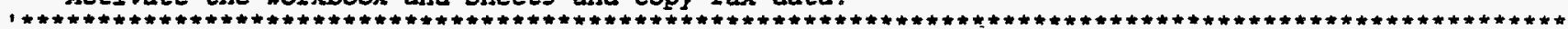
Workbooks (currentbook. Name). Activate

Worksheets ("Sheet3"). Activate

Range (Cellg (first_row, 26), Cells (last_row, 26)).Select

Selection. Copy

Celis (1, 1). Select

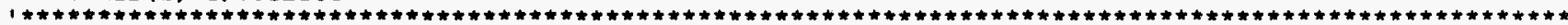
Activate the "DADS_DAT.XIS" workbook and the sheet "rax" and paste the values (paste special, values)

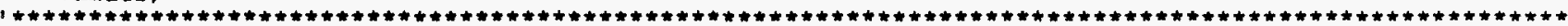
Workbooks ("DADS DAT.XIS") . Activate

Worksheets ("rax"). Activate

CeIls $(1,2)$. Select

Selection.Pastespecial Paste: $=x 1$ Values, Operation: $=x 1$ None, SkipBlanks:=False, Transpose: =False Cells (1, 1). Select

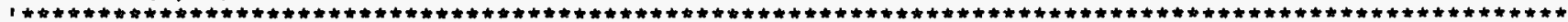
Activate the workbook and sheet 3 and copy ray data

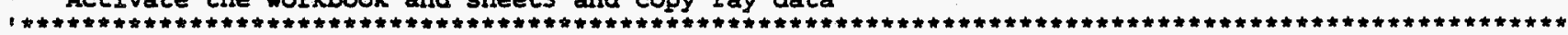
Workbooks (currentbook. Name) . Activate

Worksheets ("Sheet 3 ") . Activate

Range (Cells (Eirst_row, 27), Cells (last_row, 27)). Select

selection. Copy

Cellg (1, 1). Select

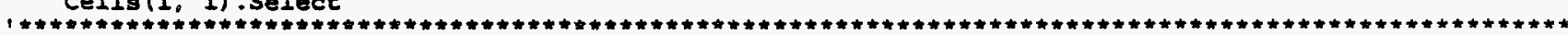
Activate the "DADS_DAT.XIS" workbook and the sheet "ray" and paste values (paste special, values)

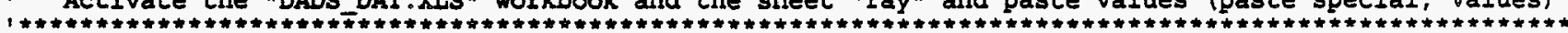
Workbooks ("DADS DAT.XIS"). Activate

Worksheets ("ray"). Activate

Cells (1, 2). Select

Selection. Pastespecial Paste: =xlValues, Operation:=xlNone, skipBlanks: =False, Transpose:=False Cells $(1,1)$. Select

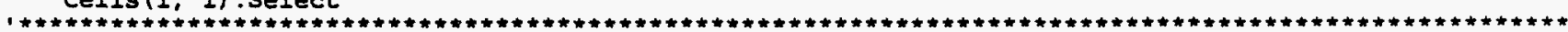
Activate the workbook and sheet 3 and copy beta data

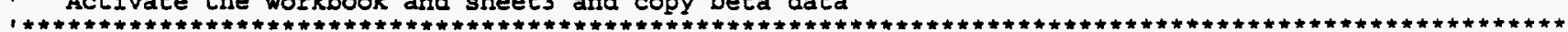
Workbooks (currentbook. Name). Activate

Worksheets ("Sheet3"). Activate

Range (Cells (first_row, 22), Cells (last_row, 22)). Select

Selection.Copy 
Cells (1, 1). Select

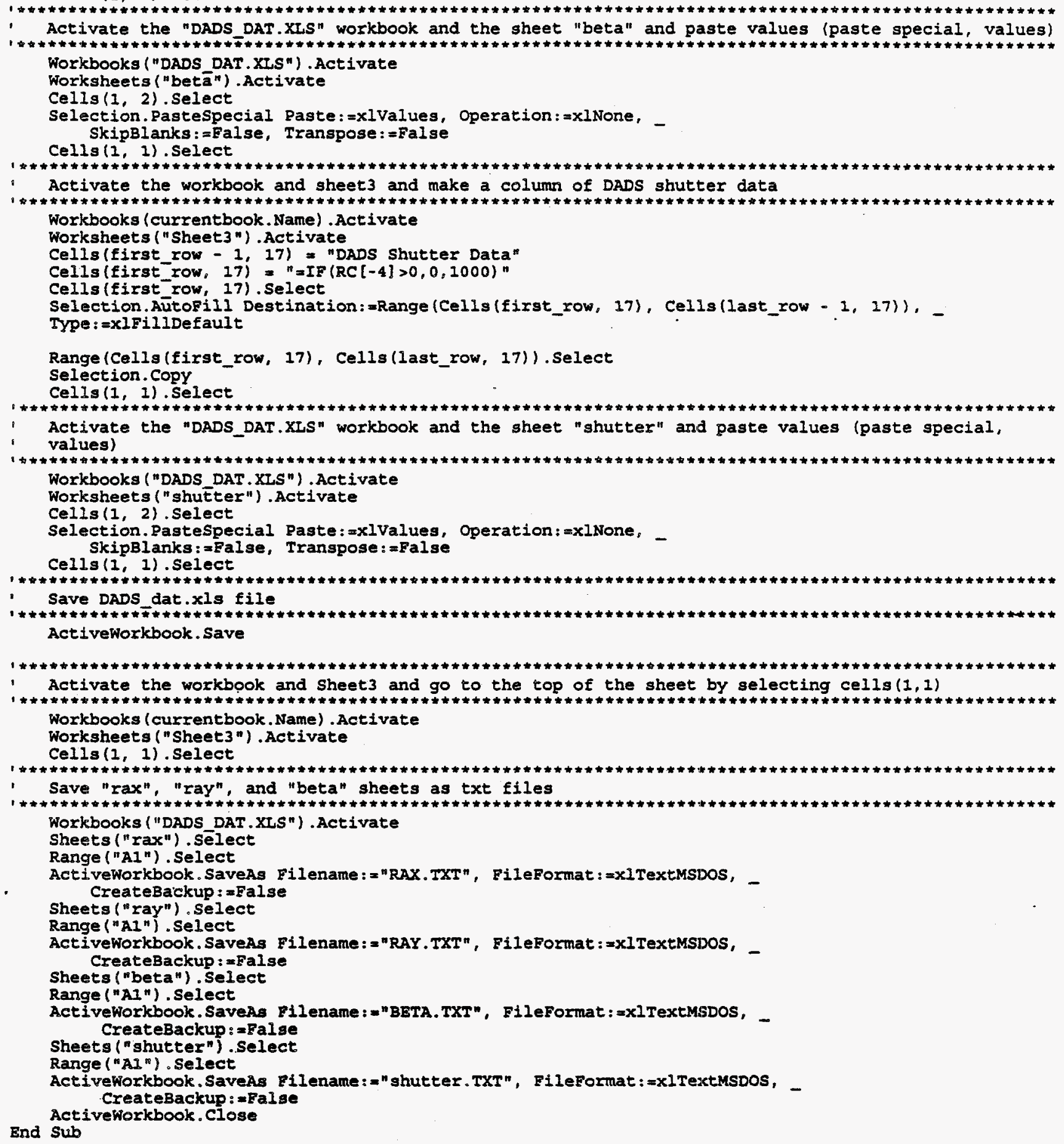

End Sub

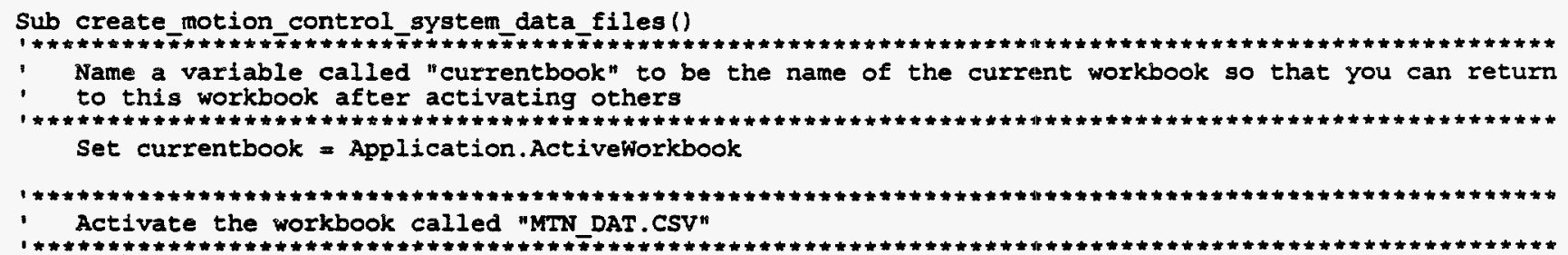


Workbooks.Open Filename: $=$ "MTN_DAT.CSV"

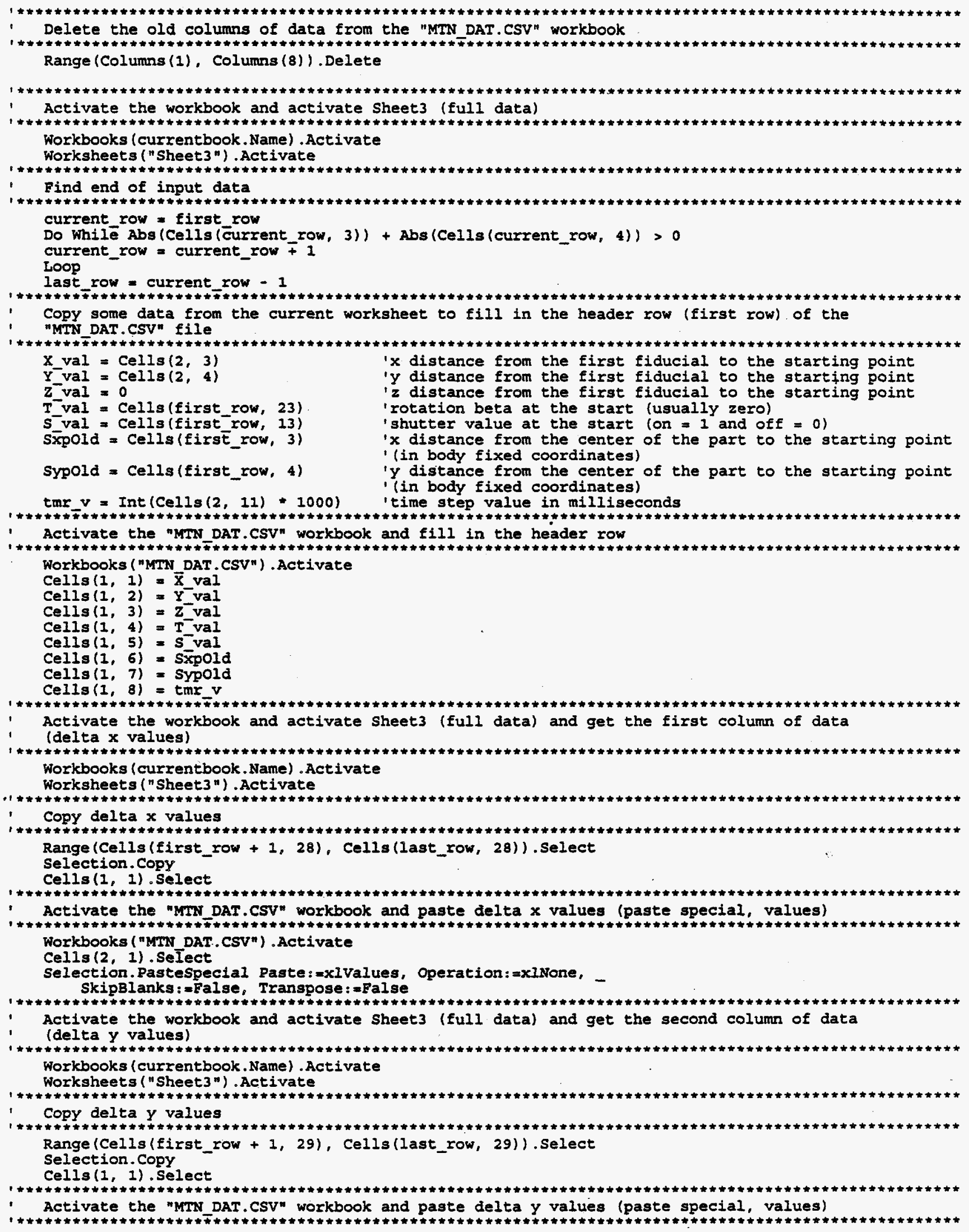


Workbooks ("MTN DAT.CSV") .Activate

Cells $(2,2)$. Select

Selection. PasteSpecial Paste: $=x$ lValues, Operation: $=x$ lNone, SkipBlanks:=False, Transpose : $=$ False

1

Fill in third column (delta $z$ ) of the "MTN_DAT.CSV" worksheet with zeros

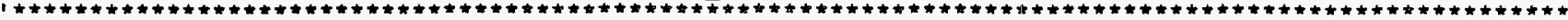

current row $=2$

For $k=1$ To last_row - first_row step 1

Cells (current row, 3 ) $=0$

current_row $=$ - current_row +1

Next $k$

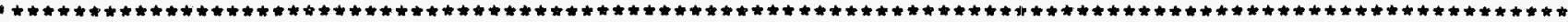
Activate the workbook and activate sheet 3 (full data) and get the fourth column of data

(delta beta values)

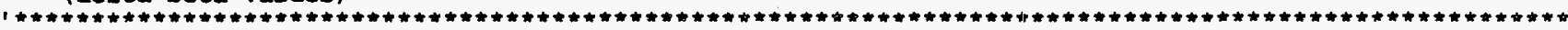
Workbooks (currentbook. Name) . Activate Worksheets ("Sheet $3 ")$. Activate

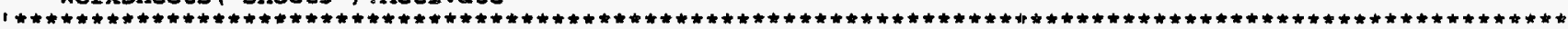
Copy delta beta values (delta beta measured in steps)

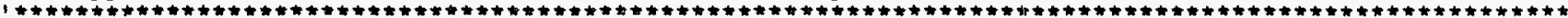
Range (Cells (firgt_row $+1,30$ ), Cells (last_row, 30)). Select Selection. Copy

Cells $(1,1)$. Select

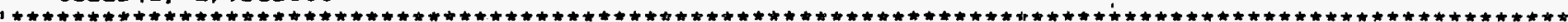
Activate the "MTN_DAT.CSV" workbook and paste delta beta values (paste special, values)

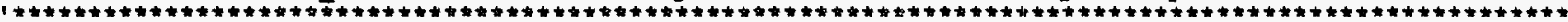
Workbooks ("MTN DAT.CSV") .Activate

Cells $(2,4)$. Select

Selection. PasteSpecial Paste:=xlValues, Operation:=xiNone, SkipBlanks : $=$ False, Transpose $:=$ False

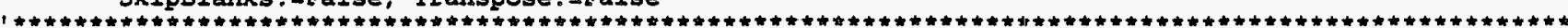
Activate the workbook and activate sheet 3 (full data) and get the fifth column of data (shutter values)

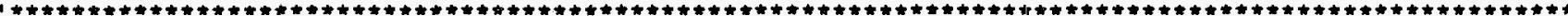
Workbooks (currentbook. Name). Activate Worksheets ("Sheet3"). Activate

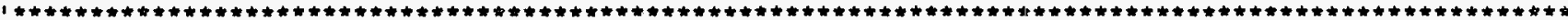
Copy shutter values (one is on and zero is off)

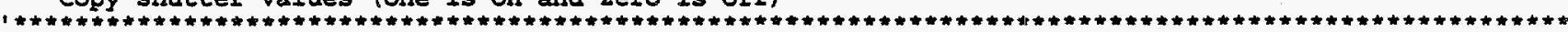
Range (Cells (first row $+1,13$ ), Cells (last row, 13)). Select

Selection. Copy Cells $(1,1)$. Select

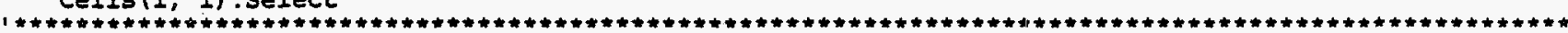
Activate the "MTN DAT.CSV" workbook and paste shutter values (paste special, values)

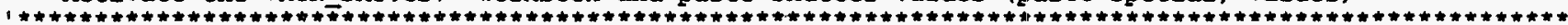
Workbooks ("MTN DAT. CSV") . Activate

Cells $(2,5)$. SeIect

Selection. PasteSpecial Paste: =xlValues, Operation: =xlNone, SkipBlanks:=False, Transpose: $=$ False

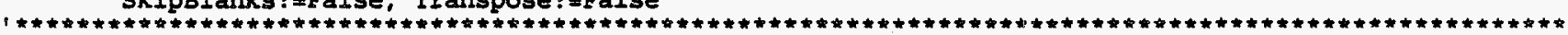
Activate the workbook and activate sheet3 (full data) and get the sixth column of data (sx' values)

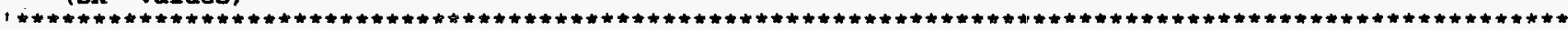
Workbooks (currentbook. Name) . Activate Worksheets ("sheet $3 "$ ) . Activate

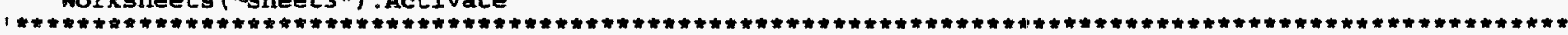
Copy sx" values

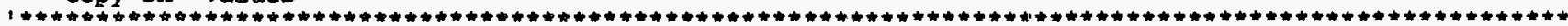
Range(Cells (first_row $+1,3)$, Cells (last_row, 3)). Select Selection. Copy Cells $(1,1)$. Select

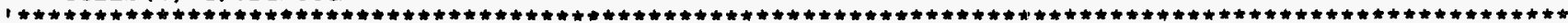
Activate the "MTN DAT.CSV" workbook and paste sx' values (paste special, values)

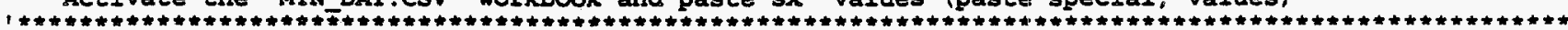
Workbooks ("MTN DAT.CSV") .Activate

CeIls $(2,6)$. Select

Selection. Pastespecial paste:=xlValues, Operation: $=x 1$ None, SkipBlanks: $=$ False, Transpose : $=$ False

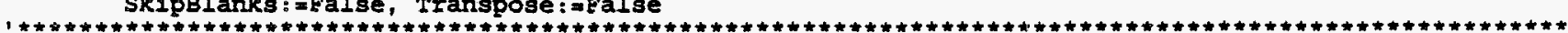
Activate the workbook and activate Sheet3 (full data) and get the seventh column of data (sy' values)

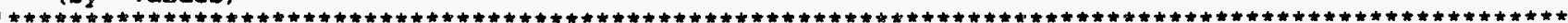
Workbooks (currentbook. Name). Activate Worksheets ("Sheet3"). Activate

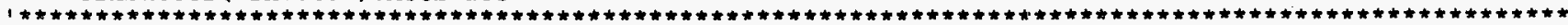
Copy sy' values

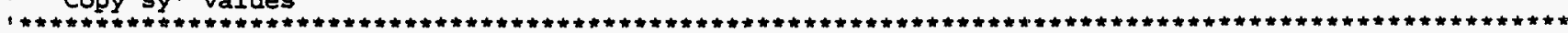
Range (Cells (first_row $+1,4)$, Cells (last_row, 4)). Select Selection. Copy 
$\operatorname{Cells}(1,1)$. Select

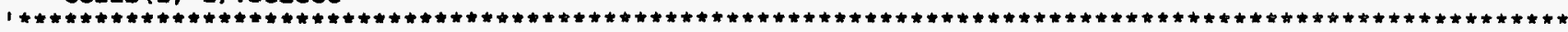
Activate the "MTN DAT.CSV" workbook and paste sy' values (paste special, values)

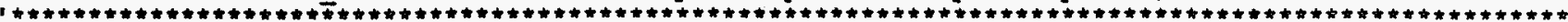
Workbooks ("MTN_DAT.CSV") .Activate

Cells (2, 7). Se Iect

Selection. Pastespecial Paste: =xlValues, Operation:=xlNone SkipBlanks : $=$ False, Transpose $:=$ False Cells $(1,1)$. Select

i

' Save the "MTN DAT.CSV" workbook as a comma delimited file and then close it

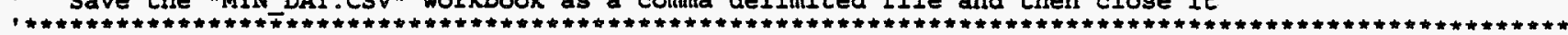
ActiveWorkbook.SaveAs Filename: = "MTN_DAT.CSV", FileFormat : =xlCSV, CreateBackup : $=$ False ActiveWorkbook. Close

1

1 Activate the workbook, activate sheet3 (full data) and go to the top of the worksheet

1

Workbooks (currentbook. Name) . Activate

Worksheets ("Sheet3"). Activate

Cells (1, 1). Select

End sub 